\title{
O-H Bond Dissociation Enthalpies in Oximes:
}

\section{Order Restored}

Derek A. Pratt, Jessie A. Blake, Peter Mulder, John C. Walton,

Hans-Gert Korth \& Keith U. Ingold

Supporting Information 
Table S1. Raw kinetic data for the thermal decomposition of $O$-benzyl oximes in tertbutylbenzene.

\begin{tabular}{|c|c|c|c|}
\hline $\mathrm{Ph}, \mathrm{Ph}$ & $\begin{array}{c}\mathrm{t}\left(10^{-3} \mathrm{~s}\right) \\
794.5\end{array}$ & $\begin{array}{c}\% \\
0.4\end{array}$ & \\
\hline initial concentration (mM): 95.8 & 1829.1 & 1.0 & \\
\hline $\mathrm{T}=170{ }^{\circ} \mathrm{C}$ & 4064.4 & 2.1 & \\
\hline & 7088.4 & 3.1 & \\
\hline & 9072 & 3.8 & \\
\hline & $10^{8} \mathrm{k} / \mathrm{s}^{-1}$ & 0.42 & \\
\hline 9-fluorenyl & $t\left(10^{-3} s\right)$ & $\%$ & \\
\hline & 622.8 & 0.3 & \\
\hline initial concentration (mM): 85.5 & 1814.4 & 1.0 & \\
\hline $\mathrm{T}=170{ }^{0} \mathrm{C}$ & 6919.8 & 4.1 & \\
\hline & $10^{8} \mathrm{k} / \mathrm{s}^{-1}$ & 0.61 & \\
\hline$i-\operatorname{Pr}, i-\operatorname{Pr}$ & $t\left(10^{-3} s\right)$ & $\%$ & \\
\hline & 1805.4 & 10.9 & \\
\hline initial concentration (mM):139.8 & 3015 & 17.7 & \\
\hline $\mathrm{T}=170{ }^{\circ} \mathrm{C}$ & 4471.2 & 27.9 & \\
\hline & 5502.6 & 40.0 & \\
\hline & $10^{8} \mathrm{k} / \mathrm{s}^{-1}$ & 8.9 & \\
\hline$t-\mathrm{Bu}, i-\mathrm{Pr}$ & $\mathrm{t}\left(10^{-3} \mathrm{~s}\right)$ & $\%$ & \\
\hline & 180 & 3.1 & \\
\hline initial concentration (mM): 131.6 & 777.9 & 7.2 & \\
\hline $\mathrm{T}=170{ }^{\circ} \mathrm{C}$ & 1292.4 & 14.7 & \\
\hline & 1972.8 & 23.5 & \\
\hline & $10^{8} \mathrm{k} / \mathrm{s}^{-1}$ & 13.2 & \\
\hline$t-B u, t-B u$ & $t\left(10^{-3} s\right)$ & $\%$ & \\
\hline & 6.9 & 0.9 & \\
\hline initial concentration (mM): 124.8 & 60.6 & 14.2 & \\
\hline $\mathrm{T}=170{ }^{\circ} \mathrm{C}$ & 75 & 16.3 & \\
\hline & 86.4 & 19.3 & \\
\hline & 148.5 & 29.0 & \\
\hline & $10^{8} \mathrm{k} / \mathrm{s}^{-1}$ & 23.7 & \\
\hline$t$-Bu,1-Ad & $t\left(10^{-3} s\right)$ & $\%$ & $\%$ \\
\hline & & Z & $E$ \\
\hline initial concentrations (mM): & 1008 & 21.5 & 11.0 \\
\hline $1.58(Z), 1.65(E)$ & 1353.6 & 31.3 & 17.3 \\
\hline $\mathrm{T}=150{ }^{\circ} \mathrm{C}$ & 1958.4 & 43.8 & 24.9 \\
\hline & $10^{8} \mathrm{k} / \mathrm{s}^{-1}$ & 34.8 & 17.7 \\
\hline
\end{tabular}


Table S2. Calculated minimum energy geometries in $O$-benzyl oxime ethers, iminoxyl and iminyl radicals (HLM: (U)B3P86/6-311G(d,p)) and imines and iminyl radicals (MLM: (U)B3LYP/6-31G(d)) from Table 3.

\begin{tabular}{cccrrr}
\multicolumn{7}{c}{ Acetone } & Oxime & Benzyl & ether - HLM & & \\
1 & 6 & 0 & 1.425366 & 3.868477 & 1.959170 \\
2 & 1 & 0 & 1.074456 & 4.036200 & 2.982940 \\
3 & 1 & 0 & 2.465950 & 3.546883 & 1.988698 \\
4 & 1 & 0 & 1.360455 & 4.828354 & 1.436509 \\
5 & 6 & 0 & 0.581362 & 2.840151 & 1.277566 \\
6 & 6 & 0 & -0.893641 & 3.064231 & 1.163036 \\
7 & 1 & 0 & -1.126330 & 4.130498 & 1.179186 \\
8 & 1 & 0 & -1.282483 & 2.609548 & 0.252229 \\
9 & 1 & 0 & -1.413233 & 2.596215 & 2.006103 \\
10 & 7 & 0 & 1.192904 & 1.806309 & 0.843122 \\
11 & 8 & 0 & 0.320690 & 0.889004 & 0.261047 \\
12 & 6 & 0 & 1.079056 & -0.133112 & -0.373680 \\
13 & 1 & 0 & 1.548101 & 0.256173 & -1.284483 \\
14 & 1 & 0 & 1.877802 & -0.446458 & 0.308618 \\
15 & 6 & 0 & 0.165782 & -1.278434 & -0.697859 \\
16 & 6 & 0 & 0.219999 & -1.895758 & -1.944667 \\
17 & 6 & 0 & -0.592496 & -2.987093 & -2.231982 \\
18 & 6 & 0 & -1.479918 & -3.463228 & -1.275824 \\
19 & 6 & 0 & -1.548736 & -2.844346 & -0.031529 \\
20 & 6 & 0 & -0.729690 & -1.761664 & 0.256609 \\
21 & 1 & 0 & 0.903266 & -1.517857 & -2.698911 \\
22 & 1 & 0 & -0.537899 & -3.458558 & -3.207017 \\
23 & 1 & 0 & -2.119252 & -4.310070 & -1.499194 \\
24 & 1 & 0 & -2.243388 & -3.208975 & 0.717326 \\
25 & 1 & 0 & -0.785795 & -1.273490 & 1.222728
\end{tabular}

Acetone Iminoxyl - HLM

$\begin{array}{cccccc}1 & 6 & 0 & 0.805573 & 1.521597 & 0.505726 \\ 2 & 1 & 0 & 0.585040 & 1.811268 & 1.538502 \\ 3 & 1 & 0 & 1.873642 & 1.322291 & 0.421024 \\ 4 & 1 & 0 & 0.558810 & 2.379294 & -0.128812 \\ 5 & 6 & 0 & 0.008588 & 0.322141 & 0.109637 \\ 6 & 6 & 0 & -1.489369 & 0.363473 & 0.147839 \\ 7 & 1 & 0 & -1.867579 & 1.155582 & -0.506401 \\ 8 & 1 & 0 & -1.913208 & -0.588196 & -0.171465 \\ 9 & 1 & 0 & -1.840861 & 0.584180 & 1.160894 \\ 10 & 7 & 0 & 0.630547 & -0.734266 & -0.260410 \\ 11 & 8 & 0 & 0.280196 & -1.845978 & -0.633760\end{array}$

\begin{tabular}{cccrrr}
\multicolumn{7}{c}{ Acetone } & Iminyl & - HLM & & & \\
1 & 6 & 0 & 0.915129 & 1.024622 & 0.332808 \\
2 & 1 & 0 & 0.949349 & 1.055639 & 1.425455 \\
3 & 1 & 0 & 1.929924 & 0.952295 & -0.055838 \\
4 & 1 & 0 & 0.462419 & 1.957864 & -0.013776 \\
5 & 6 & 0 & 0.082509 & -0.151896 & -0.122975 \\
6 & 6 & 0 & -1.358985 & -0.207499 & 0.328903 \\
7 & 1 & 0 & -1.892841 & 0.681378 & -0.018817 \\
8 & 1 & 0 & -1.851281 & -1.097074 & -0.061462 \\
9 & 1 & 0 & -1.406650 & -0.219645 & 1.421417 \\
10 & 7 & 0 & 0.568166 & -1.045974 & -0.847056
\end{tabular}

\begin{tabular}{cccccc}
\multicolumn{6}{c}{ Acetone } \\
$\begin{array}{ccccc}1 \\
1\end{array}$ & 6 & 0 & 0.845015 & 1.071365 & 0.468346 \\
2 & 1 & 0 & 1.172543 & 0.820997 & 1.485924 \\
3 & 1 & 0 & 1.720096 & 1.286153 & -0.148029 \\
4 & 1 & 0 & 0.228003 & 1.976083 & 0.547480 \\
5 & 6 & 0 & 0.055088 & -0.065770 & -0.136166 \\
6 & 6 & 0 & -1.195648 & -0.476053 & 0.615317
\end{tabular}




$\begin{array}{cccrcc}7 & 1 & 0 & -1.712532 & -1.302843 & 0.117591 \\ 8 & 1 & 0 & -0.949012 & -0.785338 & 1.639298 \\ 9 & 1 & 0 & -1.891408 & 0.368727 & 0.700857 \\ 10 & 7 & 0 & 0.479583 & -0.598237 & -1.216685 \\ 11 & 1 & 0 & -0.151497 & -1.353379 & -1.511306\end{array}$

Acetone Iminyl - MLM

$\begin{array}{cccrrr}1 & 6 & 0 & 0.919820 & 1.031056 & 0.335266 \\ 2 & 1 & 0 & 0.954401 & 1.063742 & 1.430885 \\ 3 & 1 & 0 & 1.938421 & 0.962275 & -0.052461 \\ 4 & 1 & 0 & 0.466558 & 1.967699 & -0.010985 \\ 5 & 6 & 0 & 0.083679 & -0.154050 & -0.124766 \\ 6 & 6 & 0 & -1.366946 & -0.207912 & 0.331329 \\ 7 & 1 & 0 & -1.903365 & 0.683250 & -0.016134 \\ 8 & 1 & 0 & -1.864274 & -1.098776 & -0.058074 \\ 9 & 1 & 0 & -1.416226 & -0.219373 & 1.426809 \\ 10 & 7 & 0 & 0.572167 & -1.053340 & -0.853002\end{array}$

Benzophenone Oxime Benzyl Ether - HLM

$\begin{array}{cccccc}1 & 6 & 0 & -4.627607 & 0.470318 & -3.068640 \\ 2 & 6 & 0 & -4.648946 & 0.390688 & -1.679091 \\ 3 & 6 & 0 & -3.465533 & 0.283609 & -0.966154 \\ 4 & 6 & 0 & -2.234867 & 0.252307 & -1.631935 \\ 5 & 6 & 0 & -2.223148 & 0.335750 & -3.027279 \\ 6 & 6 & 0 & -3.411495 & 0.441219 & -3.738865 \\ 7 & 1 & 0 & -5.554711 & 0.558139 & -3.624031 \\ 8 & 1 & 0 & -5.594401 & 0.419519 & -1.148697 \\ 9 & 1 & 0 & -3.473744 & 0.222735 & 0.114888 \\ 10 & 1 & 0 & -3.385043 & 0.498193 & -4.821350 \\ 11 & 6 & 0 & -0.970600 & 0.100499 & -0.875431 \\ 12 & 6 & 0 & 0.293603 & 0.626778 & -1.448833 \\ 13 & 6 & 0 & 1.444309 & -0.161690 & -1.523876 \\ 14 & 6 & 0 & 2.609431 & 0.349049 & -2.078340 \\ 15 & 6 & 0 & 2.648175 & 1.657413 & -2.547629 \\ 16 & 6 & 0 & 1.510959 & 2.452346 & -2.468226 \\ 17 & 6 & 0 & 0.338411 & 1.937271 & -1.931447 \\ 18 & 1 & 0 & 1.421712 & -1.173553 & -1.141208 \\ 19 & 1 & 0 & 3.493461 & -0.275892 & -2.137871 \\ 20 & 1 & 0 & 3.562239 & 2.055982 & -2.973564 \\ 21 & 1 & 0 & 1.534199 & 3.474678 & -2.828722 \\ 22 & 7 & 0 & -1.078216 & -0.488555 & 0.262667 \\ 23 & 8 & 0 & 0.109291 & -0.569904 & 0.957254 \\ 24 & 1 & 0 & -1.278227 & 0.306719 & -3.557022 \\ 25 & 1 & 0 & -0.551653 & 2.554449 & -1.879790 \\ 26 & 6 & 0 & -0.118511 & -1.243203 & 2.190527 \\ 27 & 1 & 0 & -0.957904 & -0.758835 & 2.704069 \\ 28 & 1 & 0 & -0.398135 & -2.284768 & 1.999006 \\ 29 & 6 & 0 & 1.130399 & -1.171369 & 3.019310 \\ 30 & 6 & 0 & 1.961681 & -0.052984 & 2.972803 \\ 31 & 6 & 0 & 3.090140 & 0.015617 & 3.779712 \\ 32 & 6 & 0 & 3.397484 & -1.026176 & 4.647546 \\ 33 & 6 & 0 & 2.573520 & -2.143970 & 4.697539 \\ 34 & 6 & 0 & 1.451096 & -2.217655 & 3.881855 \\ 35 & 1 & 0 & 1.725268 & 0.753610 & 2.289488 \\ 36 & 1 & 0 & 3.732734 & 0.887838 & 3.730216 \\ 37 & 1 & 0 & 4.278220 & -0.969817 & 5.277486 \\ 38 & 1 & 0 & 2.809886 & -2.965395 & 5.364880 \\ 39 & 1 & 0 & 0.818270 & -3.099375 & 3.914235\end{array}$

Benzophenone Iminoxyl - HLM

$\begin{array}{lllrrr}1 & 6 & 0 & -3.704175 & -0.397840 & -1.455406 \\ 2 & 6 & 0 & -3.705065 & -0.391388 & -0.064878 \\ 3 & 6 & 0 & -2.507207 & -0.399800 & 0.634600 \\ 4 & 6 & 0 & -1.286308 & -0.422342 & -0.046166 \\ 5 & 6 & 0 & -1.294761 & -0.431554 & -1.444122 \\ 6 & 6 & 0 & -2.495329 & -0.418820 & -2.141149 \\ 7 & 1 & 0 & -4.640659 & -0.385718 & -2.001402 \\ 8 & 1 & 0 & -4.643387 & -0.370318 & 0.478029 \\ 9 & 1 & 0 & -2.506748 & -0.383072 & 1.718539\end{array}$




$\begin{array}{rrrrrr}10 & 1 & 0 & -2.485199 & -0.433680 & -3.225256 \\ 11 & 6 & 0 & -0.014183 & -0.480138 & 0.703327 \\ 12 & 6 & 0 & 1.204809 & 0.235396 & 0.273498 \\ 13 & 6 & 0 & 2.478355 & -0.218743 & 0.632697 \\ 14 & 6 & 0 & 3.611374 & 0.474405 & 0.231905 \\ 15 & 6 & 0 & 3.494699 & 1.637013 & -0.521239 \\ 16 & 6 & 0 & 2.233226 & 2.103488 & -0.872036 \\ 17 & 6 & 0 & 1.096982 & 1.409595 & -0.481746 \\ 18 & 1 & 0 & 2.580814 & -1.117157 & 1.228988 \\ 19 & 1 & 0 & 4.590903 & 0.104369 & 0.512877 \\ 20 & 1 & 0 & 4.381850 & 2.179325 & -0.828082 \\ 21 & 1 & 0 & 2.131039 & 3.016236 & -1.448270 \\ 22 & 7 & 0 & -0.025823 & -1.193717 & 1.781874 \\ 23 & 8 & 0 & 0.792099 & -1.471111 & 2.641511 \\ 24 & 1 & 0 & -0.356275 & -0.465784 & -1.985400 \\ 25 & 1 & 0 & 0.117126 & 1.785068 & -0.750943\end{array}$

Benzophenone Iminyl - HLM

$\begin{array}{cccccc}1 & 6 & 0 & -3.605081 & -0.593557 & -1.268461 \\ 2 & 6 & 0 & -3.643467 & -0.530907 & 0.120444 \\ 3 & 6 & 0 & -2.463572 & -0.503914 & 0.849649 \\ 4 & 6 & 0 & -1.231306 & -0.531383 & 0.192843 \\ 5 & 6 & 0 & -1.197272 & -0.593778 & -1.201389 \\ 6 & 6 & 0 & -2.381102 & -0.627584 & -1.926714 \\ 7 & 1 & 0 & -4.528005 & -0.614184 & -1.837093 \\ 8 & 1 & 0 & -4.596144 & -0.500990 & 0.637114 \\ 9 & 1 & 0 & -2.481229 & -0.455469 & 1.931952 \\ 10 & 1 & 0 & -2.346886 & -0.681893 & -3.009013 \\ 11 & 6 & 0 & 0.030078 & -0.554190 & 0.989936 \\ 12 & 6 & 0 & 1.252247 & 0.145614 & 0.496204 \\ 13 & 6 & 0 & 2.512410 & -0.396984 & 0.757273 \\ 14 & 6 & 0 & 3.657597 & 0.268906 & 0.345217 \\ 15 & 6 & 0 & 3.556643 & 1.484494 & -0.323056 \\ 16 & 6 & 0 & 2.304838 & 2.031973 & -0.579668 \\ 17 & 6 & 0 & 1.155376 & 1.365747 & -0.175244 \\ 18 & 1 & 0 & 2.578862 & -1.344352 & 1.278921 \\ 19 & 1 & 0 & 4.632035 & -0.162699 & 0.544269 \\ 20 & 1 & 0 & 4.452482 & 2.003604 & -0.644853 \\ 21 & 1 & 0 & 2.222025 & 2.981974 & -1.095548 \\ 22 & 7 & 0 & 0.063713 & -1.168948 & 2.088279 \\ 23 & 1 & 0 & -0.243159 & -0.625472 & -1.714853 \\ 24 & 1 & 0 & 0.179692 & 1.795497 & -0.371046\end{array}$

Benzophenone Imine - MLM

\begin{tabular}{cccccc}
\multicolumn{6}{c}{ Benzophenone Imine - MLM } \\
1 & 6 & 0 & -3.720606 & -0.421216 & -1.070626 \\
2 & 6 & 0 & -3.684182 & -0.503792 & 0.324926 \\
3 & 6 & 0 & -2.464738 & -0.504303 & 0.994890 \\
4 & 6 & 0 & -1.256990 & -0.413530 & 0.281999 \\
5 & 6 & 0 & -1.304360 & -0.332752 & -1.118264 \\
6 & 6 & 0 & -2.528079 & -0.340172 & -1.789433 \\
7 & 1 & 0 & -4.673926 & -0.420446 & -1.592664 \\
8 & 1 & 0 & -4.610280 & -0.566874 & 0.890313 \\
9 & 1 & 0 & -2.420838 & -0.576317 & 2.076332 \\
10 & 1 & 0 & -2.547001 & -0.284896 & -2.874627 \\
11 & 6 & 0 & 0.035716 & -0.451576 & 1.028543 \\
12 & 6 & 0 & 1.270375 & 0.108864 & 0.388553 \\
13 & 6 & 0 & 2.476288 & -0.606347 & 0.458184 \\
14 & 6 & 0 & 3.651382 & -0.074202 & -0.070789 \\
15 & 6 & 0 & 3.641352 & 1.188387 & -0.667030 \\
16 & 6 & 0 & 2.448486 & 1.910701 & -0.738959 \\
17 & 6 & 0 & 1.269406 & 1.371834 & -0.224503 \\
18 & 1 & 0 & 2.483019 & -1.593445 & 0.913422 \\
19 & 1 & 0 & 4.573641 & -0.646765 & -0.020636 \\
20 & 1 & 0 & 4.557380 & 1.605701 & -1.076359 \\
21 & 1 & 0 & 2.435054 & 2.896138 & -1.196923 \\
22 & 7 & 0 & 0.037571 & -0.966662 & 2.206575 \\
23 & 1 & 0 & 0.973055 & -0.896271 & 2.620212 \\
24 & 1 & 0 & -0.381726 & -0.279397 & -1.687325 \\
25 & 1 & 0 & 0.344317 & 1.937823 & -0.282711
\end{tabular}




\begin{tabular}{cccrrr}
\multicolumn{6}{c}{ Benzophenone } \\
1 & 6 & 0 & -3.634473 & -0.599463 & -1.267203 \\
2 & 6 & 0 & -3.664717 & -0.552061 & 0.128685 \\
3 & 6 & 0 & -2.475738 & -0.522111 & 0.854143 \\
4 & 6 & 0 & -1.239912 & -0.532908 & 0.188846 \\
5 & 6 & 0 & -1.215684 & -0.581078 & -1.212827 \\
6 & 6 & 0 & -2.408528 & -0.616029 & -1.934983 \\
7 & 1 & 0 & -4.562611 & -0.621236 & -1.831961 \\
8 & 1 & 0 & -4.616263 & -0.535551 & 0.653110 \\
9 & 1 & 0 & -2.490703 & -0.482851 & 1.938779 \\
10 & 1 & 0 & -2.378907 & -0.657537 & -3.020446 \\
11 & 6 & 0 & 0.030255 & -0.553031 & 0.986924 \\
12 & 6 & 0 & 1.260900 & 0.149838 & 0.494764 \\
13 & 6 & 0 & 2.525772 & -0.389494 & 0.776281 \\
14 & 6 & 0 & 3.679973 & 0.275541 & 0.368384 \\
15 & 6 & 0 & 3.585838 & 1.488200 & -0.318845 \\
16 & 6 & 0 & 2.330882 & 2.032929 & -0.597195 \\
17 & 6 & 0 & 1.172528 & 1.368006 & -0.195051 \\
18 & 1 & 0 & 2.590345 & -1.333285 & 1.308530 \\
19 & 1 & 0 & 4.654235 & -0.154388 & 0.584509 \\
20 & 1 & 0 & 4.486870 & 2.005164 & -0.637835 \\
21 & 1 & 0 & 2.251693 & 2.978173 & -1.127358 \\
22 & 7 & 0 & 0.064064 & -1.172234 & 2.091963 \\
23 & 1 & 0 & -0.264052 & -0.600320 & -1.734965 \\
24 & 1 & 0 & 0.198372 & 1.797446 & -0.407647
\end{tabular}

Fluorenone Oxime Benzyl Ether - HLM

$\begin{array}{rrrrrr}1 & 6 & 0 & -3.246128 & -0.873129 & -3.307583 \\ 2 & 6 & 0 & -3.427029 & -1.399819 & -2.029703 \\ 3 & 6 & 0 & -2.399559 & -1.357211 & -1.091153 \\ 4 & 6 & 0 & -1.195466 & -0.779956 & -1.459317 \\ 5 & 6 & 0 & -1.007804 & -0.246258 & -2.745596 \\ 6 & 6 & 0 & -2.035772 & -0.291341 & -3.676671 \\ 7 & 1 & 0 & -4.059376 & -0.916501 & -4.023474 \\ 8 & 1 & 0 & -4.378622 & -1.846543 & -1.764668 \\ 9 & 1 & 0 & -2.529733 & -1.761720 & -0.094367 \\ 10 & 1 & 0 & -1.906323 & 0.116474 & -4.673190 \\ 11 & 6 & 0 & 0.047529 & -0.592207 & -0.692151 \\ 12 & 6 & 0 & 1.002121 & 0.079774 & -1.588876 \\ 13 & 6 & 0 & 2.315895 & 0.488954 & -1.399836 \\ 14 & 6 & 0 & 2.978926 & 1.113075 & -2.454880 \\ 15 & 6 & 0 & 2.340529 & 1.321461 & -3.673803 \\ 16 & 6 & 0 & 1.023877 & 0.909803 & -3.870159 \\ 17 & 6 & 0 & 0.355655 & 0.288039 & -2.827228 \\ 18 & 1 & 0 & 2.809447 & 0.319522 & -0.453676 \\ 19 & 1 & 0 & 4.004657 & 1.438634 & -2.324066 \\ 20 & 1 & 0 & 2.874946 & 1.808843 & -4.481807 \\ 21 & 1 & 0 & 0.535884 & 1.074611 & -4.824468 \\ 22 & 7 & 0 & 0.128864 & -0.987155 & 0.525609 \\ 23 & 8 & 0 & 1.360146 & -0.741988 & 1.087616 \\ 24 & 6 & 0 & 1.347391 & -1.142847 & 2.452922 \\ 25 & 1 & 0 & 0.909146 & -2.141945 & 2.528642 \\ 26 & 1 & 0 & 2.406664 & -1.207097 & 2.718100 \\ 27 & 6 & 0 & 0.630999 & -0.177508 & 3.360036 \\ 28 & 6 & 0 & 0.071911 & -0.633004 & 4.551846 \\ 29 & 6 & 0 & -0.546392 & 0.252389 & 5.425904 \\ 30 & 6 & 0 & -0.621300 & 1.603747 & 5.110132 \\ 31 & 6 & 0 & -0.073374 & 2.062477 & 3.917790 \\ 32 & 6 & 0 & 0.552567 & 1.177733 & 3.048886 \\ 33 & 1 & 0 & 0.114394 & -1.690329 & 4.795938 \\ 34 & 1 & 0 & -0.980123 & -0.115421 & 6.349275 \\ 35 & 1 & 0 & -1.110572 & 2.294988 & 5.787158 \\ 36 & 1 & 0 & -0.134674 & 3.114429 & 3.661556 \\ 37 & 1 & 0 & 0.973622 & 1.533006 & 2.115492\end{array}$

Fluorenone Iminoxyl - HLM

$\begin{array}{rrrrrr}1 & 6 & 0 & -3.442478 & 0.000002 & -0.613880 \\ 2 & 6 & 0 & -3.441844 & 0.000003 & 0.780551 \\ 3 & 6 & 0 & -2.245902 & 0.000002 & 1.491550\end{array}$




$\begin{array}{cccccc}4 & 6 & 0 & -1.055866 & 0.000001 & 0.780053 \\ 5 & 6 & 0 & -1.049429 & 0.000000 & -0.629785 \\ 6 & 6 & 0 & -2.248552 & 0.000001 & -1.329082 \\ 7 & 1 & 0 & -4.386298 & 0.000003 & -1.147306 \\ 8 & 1 & 0 & -4.384005 & 0.000004 & 1.316595 \\ 9 & 1 & 0 & -2.243191 & 0.000003 & 2.575681 \\ 10 & 1 & 0 & -2.260795 & 0.000001 & -2.413382 \\ 11 & 6 & 0 & 0.335966 & 0.000000 & 1.226567 \\ 12 & 6 & 0 & 1.189316 & -0.000001 & 0.037626 \\ 13 & 6 & 0 & 2.568855 & -0.000001 & -0.091589 \\ 14 & 6 & 0 & 3.109040 & -0.000002 & -1.375207 \\ 15 & 6 & 0 & 2.279680 & -0.000002 & -2.495222 \\ 16 & 6 & 0 & 0.893034 & -0.000001 & -2.363223 \\ 17 & 6 & 0 & 0.341626 & -0.000001 & -1.090083 \\ 18 & 1 & 0 & 3.207706 & -0.000001 & 0.784231 \\ 19 & 1 & 0 & 4.185135 & -0.000003 & -1.504430 \\ 20 & 1 & 0 & 2.721593 & -0.000003 & -3.485310 \\ 21 & 1 & 0 & 0.260589 & -0.000001 & -3.244089 \\ 22 & 7 & 0 & 0.717983 & 0.000000 & 2.458037 \\ 23 & 8 & 0 & 1.809088 & -0.000001 & 2.992762\end{array}$

Fluorenone Iminyl - HLM

$\begin{array}{rrrrrr}1 & 6 & 0 & -3.287038 & 0.000003 & -0.359427 \\ 2 & 6 & 0 & -3.291467 & 0.000004 & 1.033209 \\ 3 & 6 & 0 & -2.094201 & 0.000003 & 1.745610 \\ 4 & 6 & 0 & -0.909426 & 0.000000 & 1.032635 \\ 5 & 6 & 0 & -0.894923 & 0.000000 & -0.372129 \\ 6 & 6 & 0 & -2.091105 & 0.000001 & -1.074504 \\ 7 & 1 & 0 & -4.229350 & 0.000004 & -0.895711 \\ 8 & 1 & 0 & -4.234688 & 0.000005 & 1.567118 \\ 9 & 1 & 0 & -2.087085 & 0.000003 & 2.829270 \\ 10 & 1 & 0 & -2.101930 & 0.000001 & -2.158792 \\ 11 & 6 & 0 & 0.500522 & -0.000002 & 1.520955 \\ 12 & 6 & 0 & 1.344825 & -0.000001 & 0.291793 \\ 13 & 6 & 0 & 2.721619 & -0.000001 & 0.162746 \\ 14 & 6 & 0 & 3.262941 & -0.000001 & -1.120950 \\ 15 & 6 & 0 & 2.433107 & -0.000001 & -2.239335 \\ 16 & 6 & 0 & 1.046121 & -0.000001 & -2.105486 \\ 17 & 6 & 0 & 0.499709 & -0.000001 & -0.830534 \\ 18 & 1 & 0 & 3.358751 & -0.000001 & 1.039366 \\ 19 & 1 & 0 & 4.338984 & -0.000001 & -1.250685 \\ 20 & 1 & 0 & 2.873343 & -0.000002 & -3.230171 \\ 21 & 1 & 0 & 0.411760 & -0.000001 & -2.984908 \\ 22 & 7 & 0 & 0.889444 & -0.000001 & 2.711003\end{array}$

Fluorenone Imine - MLM

$\begin{array}{cccccc}1 & 6 & 0 & -3.316420 & 0.000006 & -0.389737 \\ 2 & 6 & 0 & -3.314978 & 0.000007 & 1.009393 \\ 3 & 6 & 0 & -2.108323 & 0.000006 & 1.718850 \\ 4 & 6 & 0 & -0.920093 & 0.000003 & 1.000622 \\ 5 & 6 & 0 & -0.915770 & 0.000002 & -0.409592 \\ 6 & 6 & 0 & -2.117084 & 0.000003 & -1.112969 \\ 7 & 1 & 0 & -4.262561 & 0.000007 & -0.924521 \\ 8 & 1 & 0 & -4.258648 & 0.000009 & 1.547853 \\ 9 & 1 & 0 & -2.088371 & 0.000007 & 2.804629 \\ 10 & 1 & 0 & -2.133054 & 0.000002 & -2.199778 \\ 11 & 6 & 0 & 0.483206 & 0.000002 & 1.492723 \\ 12 & 6 & 0 & 1.331376 & -0.000002 & 0.263187 \\ 13 & 6 & 0 & 2.713357 & -0.000006 & 0.126285 \\ 14 & 6 & 0 & 3.263519 & -0.000009 & -1.161161 \\ 15 & 6 & 0 & 2.431217 & -0.000008 & -2.284522 \\ 16 & 6 & 0 & 1.037130 & -0.000004 & -2.149409 \\ 17 & 6 & 0 & 0.488428 & -0.000002 & -0.870365 \\ 18 & 1 & 0 & 3.362474 & -0.000006 & 0.999698 \\ 19 & 1 & 0 & 4.342328 & -0.000011 & -1.288266 \\ 20 & 1 & 0 & 2.872169 & -0.000010 & -3.277768 \\ 21 & 1 & 0 & 0.402193 & -0.000004 & -3.031497 \\ 22 & 7 & 0 & 0.801102 & 0.000002 & 2.732332 \\ 23 & 1 & 0 & 1.822376 & 0.000000 & 2.843503\end{array}$


Fluorenone Iminyl - MLM

$\begin{array}{cccccc}1 & 6 & 0 & -3.301992 & 0.000003 & -0.361833 \\ 2 & 6 & 0 & -3.307045 & 0.000004 & 1.036800 \\ 3 & 6 & 0 & -2.104142 & 0.000003 & 1.752500 \\ 4 & 6 & 0 & -0.913803 & 0.000000 & 1.037766 \\ 5 & 6 & 0 & -0.898852 & -0.000001 & -0.373478 \\ 6 & 6 & 0 & -2.099678 & 0.000001 & -1.078674 \\ 7 & 1 & 0 & -4.245366 & 0.000004 & -0.901066 \\ 8 & 1 & 0 & -4.252005 & 0.000006 & 1.572526 \\ 9 & 1 & 0 & -2.098687 & 0.000003 & 2.838397 \\ 10 & 1 & 0 & -2.109614 & 0.000000 & -2.165426 \\ 11 & 6 & 0 & 0.502854 & -0.000003 & 1.528466 \\ 12 & 6 & 0 & 1.351333 & -0.000001 & 0.293379 \\ 13 & 6 & 0 & 2.733653 & -0.000001 & 0.162461 \\ 14 & 6 & 0 & 3.277539 & -0.000001 & -1.127236 \\ 15 & 6 & 0 & 2.443688 & -0.000001 & -2.250112 \\ 16 & 6 & 0 & 1.050521 & -0.000002 & -2.113911 \\ 17 & 6 & 0 & 0.502060 & -0.000002 & -0.833924 \\ 18 & 1 & 0 & 3.373438 & -0.000001 & 1.039904 \\ 19 & 1 & 0 & 4.356063 & -0.000001 & -1.256521 \\ 20 & 1 & 0 & 2.883084 & -0.000001 & -3.243925 \\ 21 & 1 & 0 & 0.413951 & -0.000002 & -2.994763 \\ 22 & 7 & 0 & 0.894618 & -0.000001 & 2.725377\end{array}$

Di-iso-propyl ketone Benzyl Ether - HLM

$\begin{array}{rrrrrr}1 & 6 & 0 & -2.852271 & 0.239259 & 1.966745 \\ 2 & 1 & 0 & -2.784796 & 0.295828 & 3.058597 \\ 3 & 6 & 0 & -1.436710 & 0.054545 & 1.441393 \\ 4 & 6 & 0 & -0.284707 & -0.071256 & 2.410098 \\ 5 & 1 & 0 & 0.613703 & -0.235796 & 1.813966 \\ 6 & 7 & 0 & -1.334105 & 0.020975 & 0.169911 \\ 7 & 8 & 0 & -0.025166 & -0.133227 & -0.282260 \\ 8 & 6 & 0 & -0.100140 & 1.222873 & 3.208124 \\ 9 & 1 & 0 & -0.975008 & 1.447486 & 3.825692 \\ 10 & 1 & 0 & 0.759747 & 1.131391 & 3.877439 \\ 11 & 1 & 0 & 0.076622 & 2.074824 & 2.547488 \\ 12 & 6 & 0 & -0.469907 & -1.275123 & 3.337482 \\ 13 & 1 & 0 & -1.358289 & -1.172750 & 3.967639 \\ 14 & 1 & 0 & -0.561131 & -2.203919 & 2.769530 \\ 15 & 1 & 0 & 0.393809 & -1.371084 & 4.001141 \\ 16 & 6 & 0 & -3.468909 & 1.545821 & 1.464703 \\ 17 & 1 & 0 & -3.542981 & 1.532146 & 0.375442 \\ 18 & 1 & 0 & -4.470964 & 1.683905 & 1.880881 \\ 19 & 1 & 0 & -2.861397 & 2.408888 & 1.749079 \\ 20 & 6 & 0 & -3.733406 & -0.959852 & 1.609894 \\ 21 & 1 & 0 & -4.738716 & -0.836867 & 2.023010 \\ 22 & 1 & 0 & -3.812432 & -1.054927 & 0.524797 \\ 23 & 1 & 0 & -3.318626 & -1.892879 & 1.999944 \\ 24 & 6 & 0 & -0.044340 & -0.299263 & -1.695272 \\ 25 & 1 & 0 & -0.476030 & -1.272706 & -1.953409 \\ 26 & 1 & 0 & -0.682287 & 0.479078 & -2.130013 \\ 27 & 6 & 0 & 1.362092 & -0.192041 & -2.205714 \\ 28 & 6 & 0 & 1.883191 & -1.159732 & -3.060123 \\ 29 & 6 & 0 & 3.174296 & -1.040903 & -3.563274 \\ 30 & 6 & 0 & 3.962559 & 0.044207 & -3.203718 \\ 31 & 6 & 0 & 3.453276 & 1.010696 & -2.341642 \\ 32 & 6 & 0 & 2.161270 & 0.894862 & -1.848965 \\ 33 & 1 & 0 & 1.273563 & -2.015510 & -3.332779 \\ 34 & 1 & 0 & 3.566351 & -1.801698 & -4.229156 \\ 35 & 1 & 0 & 4.971496 & 0.136488 & -3.590140 \\ 36 & 1 & 0 & 4.065957 & 1.858494 & -2.055198 \\ 37 & 1 & 0 & 1.763717 & 1.644034 & -1.173628\end{array}$

Di-iso-propyl Ketone Iminoxyl - HLM

$\begin{array}{rrrrrr}1 & 6 & 0 & -1.317585 & -0.054533 & 0.161930 \\ 2 & 1 & 0 & -1.312054 & -0.066581 & 1.257549 \\ 3 & 6 & 0 & 0.131628 & -0.060799 & -0.274433 \\ 4 & 6 & 0 & 1.268826 & 0.181761 & 0.699694 \\ 5 & 1 & 0 & 2.181114 & 0.223180 & 0.096586 \\ 6 & 7 & 0 & 0.386313 & -0.284941 & -1.508773\end{array}$




$\begin{array}{cccccc}7 & 8 & 0 & 1.406005 & -0.352681 & -2.183606 \\ 8 & 6 & 0 & 1.115956 & 1.514261 & 1.433916 \\ 9 & 1 & 0 & 0.210459 & 1.534384 & 2.047594 \\ 10 & 1 & 0 & 1.967162 & 1.674041 & 2.101553 \\ 11 & 1 & 0 & 1.073711 & 2.353296 & 0.735744 \\ 12 & 6 & 0 & 1.398403 & -0.984433 & 1.683344 \\ 13 & 1 & 0 & 0.503050 & -1.085512 & 2.303946 \\ 14 & 1 & 0 & 1.557673 & -1.929285 & 1.159262 \\ 15 & 1 & 0 & 2.246618 & -0.820973 & 2.354055 \\ 16 & 6 & 0 & -2.030023 & 1.223867 & -0.291253 \\ 17 & 1 & 0 & -2.062701 & 1.270234 & -1.383189 \\ 18 & 1 & 0 & -3.057863 & 1.247365 & 0.081906 \\ 19 & 1 & 0 & -1.518466 & 2.120007 & 0.067144 \\ 20 & 6 & 0 & -2.063607 & -1.298082 & -0.321173 \\ 21 & 1 & 0 & -3.099613 & -1.286788 & 0.028105 \\ 22 & 1 & 0 & -2.073267 & -1.336379 & -1.413513 \\ 23 & 1 & 0 & -1.589638 & -2.213197 & 0.041372\end{array}$

Di-iso-propyl ketone Iminyl - HLM

$\begin{array}{cccrrr}1 & 6 & 0 & -1.137205 & -0.068247 & -0.099383 \\ 2 & 1 & 0 & -1.109754 & -0.006008 & 0.994107 \\ 3 & 6 & 0 & 0.315618 & -0.033017 & -0.595142 \\ 4 & 6 & 0 & 1.450954 & 0.104270 & 0.424304 \\ 5 & 1 & 0 & 2.376464 & 0.117059 & -0.158604 \\ 6 & 7 & 0 & 0.585874 & -0.114122 & -1.808935 \\ 7 & 6 & 0 & 1.340634 & 1.422376 & 1.189493 \\ 8 & 1 & 0 & 0.422674 & 1.469776 & 1.782548 \\ 9 & 1 & 0 & 2.182667 & 1.525481 & 1.879526 \\ 10 & 1 & 0 & 1.354154 & 2.278271 & 0.511109 \\ 11 & 6 & 0 & 1.485381 & -1.101126 & 1.363106 \\ 12 & 1 & 0 & 0.575663 & -1.169383 & 1.966629 \\ 13 & 1 & 0 & 1.597730 & -2.034498 & 0.807184 \\ 14 & 1 & 0 & 2.330247 & -1.014584 & 2.051948 \\ 15 & 6 & 0 & -1.910932 & 1.136633 & -0.633507 \\ 16 & 1 & 0 & -1.947690 & 1.109787 & -1.724912 \\ 17 & 1 & 0 & -2.935765 & 1.130929 & -0.251867 \\ 18 & 1 & 0 & -1.444050 & 2.079024 & -0.337341 \\ 19 & 6 & 0 & -1.806147 & -1.384047 & -0.493720 \\ 20 & 1 & 0 & -2.831136 & -1.419030 & -0.114142 \\ 21 & 1 & 0 & -1.835787 & -1.483443 & -1.581081 \\ 22 & 1 & 0 & -1.266352 & -2.245578 & -0.093476\end{array}$

Di-iso-propyl Ketone Imine - MLM

$\begin{array}{rrrrrr}1 & 6 & 0 & -1.147063 & -0.029353 & -0.074470 \\ 2 & 1 & 0 & -1.147940 & -0.000905 & 1.022693 \\ 3 & 6 & 0 & 0.305391 & -0.008585 & -0.564062 \\ 4 & 6 & 0 & 1.425073 & 0.046782 & 0.475068 \\ 5 & 1 & 0 & 2.370803 & 0.054782 & -0.086331 \\ 6 & 7 & 0 & 0.498283 & -0.039212 & -1.826514 \\ 7 & 1 & 0 & 1.503085 & -0.021032 & -2.038339 \\ 8 & 6 & 0 & 1.367453 & 1.339588 & 1.311140 \\ 9 & 1 & 0 & 0.451638 & 1.391676 & 1.910947 \\ 10 & 1 & 0 & 2.217160 & 1.382063 & 2.002484 \\ 11 & 1 & 0 & 1.405708 & 2.229385 & 0.673127 \\ 12 & 6 & 0 & 1.429123 & -1.200870 & 1.379021 \\ 13 & 1 & 0 & 0.516649 & -1.264516 & 1.982812 \\ 14 & 1 & 0 & 1.510038 & -2.120578 & 0.789242 \\ 15 & 1 & 0 & 2.279694 & -1.165719 & 2.069714 \\ 16 & 6 & 0 & -1.905255 & 1.208745 & -0.586263 \\ 17 & 1 & 0 & -1.898920 & 1.224381 & -1.680276 \\ 18 & 1 & 0 & -2.945423 & 1.194216 & -0.239769 \\ 19 & 1 & 0 & -1.444526 & 2.138650 & -0.233081 \\ 20 & 6 & 0 & -1.847210 & -1.325590 & -0.520597 \\ 21 & 1 & 0 & -2.887653 & -1.340318 & -0.174912 \\ 22 & 1 & 0 & -1.837969 & -1.398047 & -1.612285 \\ 23 & 1 & 0 & -1.345394 & -2.213849 & -0.119456\end{array}$




\begin{tabular}{cccrrr}
\multicolumn{6}{c}{ Di-iso-propyl Ketone Iminyl - MLM } \\
1 & 6 & 0 & -1.149436 & -0.064944 & -0.105280 \\
2 & 1 & 0 & -1.132259 & 0.004958 & 0.989448 \\
3 & 6 & 0 & 0.315050 & -0.023955 & -0.596672 \\
4 & 6 & 0 & 1.458518 & 0.101311 & 0.430638 \\
5 & 1 & 0 & 2.386707 & 0.110925 & -0.152049 \\
6 & 7 & 0 & 0.591388 & -0.090892 & -1.817777 \\
7 & 6 & 0 & 1.364783 & 1.425894 & 1.206937 \\
8 & 1 & 0 & 0.452046 & 1.479933 & 1.811827 \\
9 & 1 & 0 & 2.217727 & 1.520924 & 1.889169 \\
10 & 1 & 0 & 1.377005 & 2.285733 & 0.528822 \\
11 & 6 & 0 & 1.494463 & -1.115025 & 1.371517 \\
12 & 1 & 0 & 0.588096 & -1.182834 & 1.984546 \\
13 & 1 & 0 & 1.597825 & -2.049251 & 0.809521 \\
14 & 1 & 0 & 2.348344 & -1.034762 & 2.054474 \\
15 & 6 & 0 & -1.937481 & 1.136876 & -0.652988 \\
16 & 1 & 0 & -1.973114 & 1.105824 & -1.746838 \\
17 & 1 & 0 & -2.965620 & 1.123784 & -0.272454 \\
18 & 1 & 0 & -1.479192 & 2.087455 & -0.359035 \\
19 & 6 & 0 & -1.813437 & -1.396774 & -0.493246 \\
20 & 1 & 0 & -2.840420 & -1.436033 & -0.111402 \\
21 & 1 & 0 & -1.844809 & -1.506904 & -1.582102 \\
22 & 1 & 0 & -1.266807 & -2.253813 & -0.084926
\end{tabular}

Iso-propyl Tert-butyl Ketone Oxime Ether - HLM

$\begin{array}{cccccc}1 & 6 & 0 & 3.197954 & -0.654269 & 0.040398 \\ 2 & 6 & 0 & 1.798439 & -0.028694 & 0.052073 \\ 3 & 6 & 0 & 1.503514 & 1.464577 & 0.059486 \\ 4 & 7 & 0 & 0.844174 & -0.881666 & 0.003225 \\ 5 & 8 & 0 & -0.421160 & -0.299876 & -0.012518 \\ 6 & 6 & 0 & 2.449135 & 2.373888 & 0.841442 \\ 7 & 1 & 0 & 2.514836 & 2.086552 & 1.892784 \\ 8 & 1 & 0 & 3.460684 & 2.404760 & 0.431185 \\ 9 & 1 & 0 & 2.057527 & 3.394713 & 0.807414 \\ 10 & 6 & 0 & 3.118933 & -2.160738 & -0.233544 \\ 11 & 1 & 0 & 2.632211 & -2.368195 & -1.188073 \\ 12 & 1 & 0 & 4.133241 & -2.570228 & -0.262674 \\ 13 & 1 & 0 & 2.555562 & -2.681018 & 0.541636 \\ 14 & 6 & 0 & 4.057717 & -0.021438 & -1.064588 \\ 15 & 1 & 0 & 5.047026 & -0.488502 & -1.067280 \\ 16 & 1 & 0 & 3.610458 & -0.182047 & -2.049099 \\ 17 & 1 & 0 & 4.201689 & 1.051381 & -0.926836 \\ 18 & 6 & 0 & 1.307730 & 1.966611 & -1.379077 \\ 19 & 1 & 0 & 2.240861 & 1.947482 & -1.947018 \\ 20 & 1 & 0 & 0.569724 & 1.355676 & -1.901135 \\ 21 & 1 & 0 & 0.944183 & 2.998186 & -1.366336 \\ 22 & 6 & 0 & 3.885693 & -0.468575 & 1.404636 \\ 23 & 1 & 0 & 4.099025 & 0.574794 & 1.634272 \\ 24 & 1 & 0 & 3.267531 & -0.878669 & 2.207676 \\ 25 & 1 & 0 & 4.837585 & -1.008274 & 1.406059 \\ 26 & 1 & 0 & 0.525788 & 1.542653 & 0.541957 \\ 27 & 6 & 0 & -1.399275 & -1.339874 & -0.016112 \\ 28 & 1 & 0 & -1.282283 & -1.945928 & -0.920142 \\ 29 & 1 & 0 & -1.238389 & -1.984146 & 0.854562 \\ 30 & 6 & 0 & -2.750171 & -0.693338 & 0.030257 \\ 31 & 6 & 0 & -3.268253 & -0.230832 & 1.239729 \\ 32 & 6 & 0 & -4.507531 & 0.393150 & 1.286210 \\ 33 & 6 & 0 & -5.245218 & 0.562173 & 0.119153 \\ 34 & 6 & 0 & -4.737523 & 0.106560 & -1.091249 \\ 35 & 6 & 0 & -3.494851 & -0.514706 & -1.133241 \\ 36 & 1 & 0 & -2.692702 & -0.364062 & 2.150093 \\ 37 & 1 & 0 & -4.901169 & 0.745318 & 2.233284 \\ 38 & 1 & 0 & -6.215093 & 1.045887 & 0.154454 \\ 39 & 1 & 0 & -5.309361 & 0.235176 & -2.003547 \\ 40 & 1 & 0 & -3.096640 & -0.867809 & -2.079100\end{array}$




\begin{tabular}{crrrrr}
\multicolumn{6}{l}{ Iso-propyl Tert-butyl Ketone Iminoxyl - HLM } \\
1 & 6 & 0 & 1.243576 & -0.170899 & 0.079412 \\
2 & 6 & 0 & -0.151922 & 0.402702 & -0.146425 \\
3 & 6 & 0 & -1.472799 & -0.362033 & -0.224744 \\
4 & 7 & 0 & -0.248296 & 1.680501 & -0.223100 \\
5 & 8 & 0 & -1.184749 & 2.453789 & -0.380511 \\
6 & 6 & 0 & -1.431502 & -1.719121 & -0.921231 \\
7 & 1 & 0 & -1.043185 & -1.643504 & -1.938689 \\
8 & 1 & 0 & -0.834602 & -2.455532 & -0.378678 \\
9 & 1 & 0 & -2.449216 & -2.114393 & -0.985652 \\
10 & 6 & 0 & 2.231649 & 0.964135 & 0.370340 \\
11 & 1 & 0 & 1.941821 & 1.529785 & 1.258792 \\
12 & 1 & 0 & 3.227304 & 0.545134 & 0.539842 \\
13 & 1 & 0 & 2.294263 & 1.662885 & -0.466727 \\
14 & 6 & 0 & 1.243852 & -1.127397 & 1.281484 \\
15 & 1 & 0 & 2.253956 & -1.516163 & 1.439899 \\
16 & 1 & 0 & 0.935427 & -0.612079 & 2.194796 \\
17 & 1 & 0 & 0.582818 & -1.983289 & 1.132940 \\
18 & 6 & 0 & -2.122198 & -0.459355 & 1.161419 \\
19 & 1 & 0 & -1.550565 & -1.106725 & 1.830350 \\
20 & 1 & 0 & -2.203392 & 0.527086 & 1.623106 \\
21 & 1 & 0 & -3.131017 & -0.873577 & 1.075044 \\
22 & 6 & 0 & 1.738751 & -0.916674 & -1.172024 \\
23 & 1 & 0 & 1.140175 & -1.799205 & -1.398185 \\
24 & 1 & 0 & 1.724695 & -0.260088 & -2.045787 \\
25 & 1 & 0 & 2.769734 & -1.248387 & -1.016039 \\
26 & 1 & 0 & -2.118591 & 0.286081 & -0.828604
\end{tabular}

Iso-propyl Tert-butyl Ketone Iminyl - HLM

$\begin{array}{ccrrrr}\text { Iso-propyl Trt-butyl Ketone lminyl }- \text { HLM } \\ 1 & 6 & 0 & -0.563097 & -0.020070 & -0.961584 \\ 2 & 6 & 0 & -0.540264 & 0.048278 & 0.584941 \\ 3 & 6 & 0 & 0.721315 & -0.045883 & 1.466007 \\ 4 & 7 & 0 & -1.618737 & 0.180479 & 1.201986 \\ 5 & 6 & 0 & 1.643105 & -1.223698 & 1.154506 \\ 6 & 1 & 0 & 1.104327 & -2.173516 & 1.166914 \\ 7 & 1 & 0 & 2.139020 & -1.122247 & 0.186277 \\ 8 & 1 & 0 & 2.426033 & -1.276689 & 1.916472 \\ 9 & 6 & 0 & -1.887047 & 0.566951 & -1.464280 \\ 10 & 1 & 0 & -1.985900 & 1.617091 & -1.180472 \\ 11 & 1 & 0 & -1.927793 & 0.498825 & -2.555414 \\ 12 & 1 & 0 & -2.741851 & 0.028835 & -1.051019 \\ 13 & 6 & 0 & 0.590225 & 0.761181 & -1.596050 \\ 14 & 1 & 0 & 0.499764 & 0.705878 & -2.685180 \\ 15 & 1 & 0 & 0.561879 & 1.816685 & -1.315153 \\ 16 & 1 & 0 & 1.568532 & 0.357748 & -1.328776 \\ 17 & 6 & 0 & 1.485509 & 1.280680 & 1.516251 \\ 18 & 1 & 0 & 2.003212 & 1.500450 & 0.581103 \\ 19 & 1 & 0 & 0.813606 & 2.111652 & 1.741075 \\ 20 & 1 & 0 & 2.239226 & 1.235662 & 2.307719 \\ 21 & 6 & 0 & -0.490756 & -1.494418 & -1.389254 \\ 22 & 1 & 0 & 0.454133 & -1.963055 & -1.111678 \\ 23 & 1 & 0 & -1.305067 & -2.068489 & -0.941134 \\ 24 & 1 & 0 & -0.587960 & -1.559201 & -2.477408 \\ 25 & 1 & 0 & 0.316053 & -0.211112 & 2.469553\end{array}$

\begin{tabular}{cccrrr}
\multicolumn{7}{l}{ Iso-propyl Tert-butyl Ketone Imine - MLM } \\
1 & 6 & 0 & 1.091230 & -0.186461 & 0.111917 \\
2 & 6 & 0 & -0.094647 & 0.706694 & -0.331535 \\
3 & 6 & 0 & -1.571081 & 0.256942 & -0.265382 \\
4 & 7 & 0 & 0.207602 & 1.869851 & -0.771591 \\
5 & 1 & 0 & -0.636472 & 2.370124 & -1.075088 \\
6 & 6 & 0 & -1.904901 & -1.131172 & -0.841586 \\
7 & 1 & 0 & -1.539307 & -1.240757 & -1.867874 \\
8 & 1 & 0 & -1.492861 & -1.949994 & -0.244164 \\
9 & 1 & 0 & -2.993666 & -1.260757 & -0.862130 \\
10 & 6 & 0 & 2.331987 & 0.690503 & 0.379643 \\
11 & 1 & 0 & 2.140758 & 1.410527 & 1.182130 \\
12 & 1 & 0 & 3.172970 & 0.053543 & 0.680414 \\
13 & 1 & 0 & 2.617177 & 1.259314 & -0.507076
\end{tabular}




$\begin{array}{rrrrrr}14 & 6 & 0 & 0.799055 & -0.995393 & 1.393960 \\ 15 & 1 & 0 & 1.683374 & -1.588340 & 1.655745 \\ 16 & 1 & 0 & 0.583404 & -0.336761 & 2.242746 \\ 17 & 1 & 0 & -0.035814 & -1.692754 & 1.281822 \\ 18 & 6 & 0 & -2.170171 & 0.433684 & 1.147343 \\ 19 & 1 & 0 & -1.787679 & -0.304890 & 1.856665 \\ 20 & 1 & 0 & -1.953396 & 1.431047 & 1.545122 \\ 21 & 1 & 0 & -3.259646 & 0.317332 & 1.102952 \\ 22 & 6 & 0 & 1.438724 & -1.161000 & -1.042671 \\ 23 & 1 & 0 & 0.635590 & -1.872922 & -1.251979 \\ 24 & 1 & 0 & 1.654146 & -0.606924 & -1.962730 \\ 25 & 1 & 0 & 2.332373 & -1.739862 & -0.779127 \\ 26 & 1 & 0 & -2.095343 & 0.980348 & -0.906420\end{array}$

Iso-propyl Tert-butyl Ketone Iminyl - MLM

$\begin{array}{rrrrrr}1 & 6 & 0 & -0.567544 & -0.019936 & -0.970214 \\ 2 & 6 & 0 & -0.541129 & 0.038983 & 0.588169 \\ 3 & 6 & 0 & 0.729207 & -0.040360 & 1.477515 \\ 4 & 7 & 0 & -1.627468 & 0.143825 & 1.211398 \\ 5 & 6 & 0 & 1.664976 & -1.222041 & 1.173664 \\ 6 & 1 & 0 & 1.128219 & -2.176151 & 1.189238 \\ 7 & 1 & 0 & 2.164863 & -1.125042 & 0.204459 \\ 8 & 1 & 0 & 2.447298 & -1.267731 & 1.940729 \\ 9 & 6 & 0 & -1.897294 & 0.583831 & -1.469982 \\ 10 & 1 & 0 & -1.988501 & 1.635943 & -1.180493 \\ 11 & 1 & 0 & -1.942711 & 0.523385 & -2.563919 \\ 12 & 1 & 0 & -2.758286 & 0.049581 & -1.058327 \\ 13 & 6 & 0 & 0.594535 & 0.760663 & -1.613749 \\ 14 & 1 & 0 & 0.497993 & 0.711453 & -2.705199 \\ 15 & 1 & 0 & 0.576824 & 1.817792 & -1.328141 \\ 16 & 1 & 0 & 1.574527 & 0.349714 & -1.354613 \\ 17 & 6 & 0 & 1.490888 & 1.298969 & 1.528313 \\ 18 & 1 & 0 & 2.011424 & 1.522666 & 0.592915 \\ 19 & 1 & 0 & 0.811475 & 2.128295 & 1.750702 \\ 20 & 1 & 0 & 2.244261 & 1.258876 & 2.324225 \\ 21 & 6 & 0 & -0.507606 & -1.502109 & -1.409953 \\ 22 & 1 & 0 & 0.434806 & -1.982076 & -1.132858 \\ 23 & 1 & 0 & -1.328307 & -2.073797 & -0.964191 \\ 24 & 1 & 0 & -0.603930 & -1.563114 & -2.500996 \\ 25 & 1 & 0 & 0.326127 & -0.204576 & 2.484117\end{array}$

Di-tert-butyl Ketone Oxime Ether - HLM

$\begin{array}{cccrrr}\text { Di-tert-butyl Ketone Oxime Ether - } & \\ 1 & 6 & 0 & -3.120296 & -0.863206 & 0.029059 \\ 2 & 6 & 0 & -1.779532 & -0.097812 & -0.053532 \\ 3 & 6 & 0 & -1.625785 & 1.441059 & -0.056218 \\ 4 & 7 & 0 & -0.784884 & -0.906586 & -0.046482 \\ 5 & 8 & 0 & 0.479371 & -0.331695 & -0.036342 \\ 6 & 6 & 0 & -2.922742 & 2.256685 & -0.168707 \\ 7 & 1 & 0 & -3.663713 & 2.016273 & 0.593669 \\ 8 & 1 & 0 & -2.660876 & 3.310886 & -0.038756 \\ 9 & 1 & 0 & -3.386309 & 2.163264 & -1.151315 \\ 10 & 6 & 0 & -0.946915 & 1.852892 & 1.264539 \\ 11 & 1 & 0 & -1.592661 & 1.629338 & 2.118560 \\ 12 & 1 & 0 & 0.004583 & 1.342682 & 1.401475 \\ 13 & 1 & 0 & -0.763490 & 2.931832 & 1.257518 \\ 14 & 6 & 0 & -4.097253 & -0.468127 & -1.091893 \\ 15 & 1 & 0 & -3.608223 & -0.500451 & -2.069649 \\ 16 & 1 & 0 & -4.921950 & -1.186374 & -1.113858 \\ 17 & 1 & 0 & -4.534492 & 0.518590 & -0.957383 \\ 18 & 6 & 0 & -2.891712 & -2.376415 & -0.102253 \\ 19 & 1 & 0 & -3.858688 & -2.882989 & -0.026213 \\ 20 & 1 & 0 & -2.442349 & -2.630723 & -1.063836 \\ 21 & 1 & 0 & -2.235401 & -2.757920 & 0.679013 \\ 22 & 6 & 0 & -0.764398 & 1.877051 & -1.258835 \\ 23 & 1 & 0 & 0.237920 & 1.460462 & -1.221502 \\ 24 & 1 & 0 & -1.235387 & 1.571332 & -2.197670 \\ 25 & 1 & 0 & -0.691010 & 2.968925 & -1.264977 \\ 26 & 6 & 0 & -3.762355 & -0.628321 & 1.407991 \\ 27 & 1 & 0 & -3.100857 & -0.985556 & 2.201578\end{array}$




$\begin{array}{rrrrrr}28 & 1 & 0 & -3.991843 & 0.419029 & 1.605503 \\ 29 & 1 & 0 & -4.699943 & -1.188634 & 1.474157 \\ 30 & 6 & 0 & 1.447657 & -1.382847 & -0.075478 \\ 31 & 1 & 0 & 1.282652 & -2.049687 & 0.776992 \\ 32 & 1 & 0 & 1.315521 & -1.960127 & -0.995787 \\ 33 & 6 & 0 & 2.807079 & -0.755308 & -0.022213 \\ 34 & 6 & 0 & 3.357194 & -0.363812 & 1.198104 \\ 35 & 6 & 0 & 4.605640 & 0.241696 & 1.249785 \\ 36 & 6 & 0 & 5.320570 & 0.462132 & 0.077408 \\ 37 & 6 & 0 & 4.781842 & 0.075640 & -1.143748 \\ 38 & 6 & 0 & 3.530725 & -0.527572 & -1.190873 \\ 39 & 1 & 0 & 2.800535 & -0.538783 & 2.113190 \\ 40 & 1 & 0 & 5.023852 & 0.539053 & 2.205025 \\ 41 & 1 & 0 & 6.297317 & 0.931445 & 0.116598 \\ 42 & 1 & 0 & 5.336511 & 0.243490 & -2.060236 \\ 43 & 1 & 0 & 3.109204 & -0.828099 & -2.144796\end{array}$

Di-tert-butyl Iminoxyl - HLM

\begin{tabular}{cccrrr}
\multicolumn{1}{c}{ i-tert-butyl Iminoxyl-HL } & & & \\
2 & 6 & 0 & 1.491635 & -0.109398 & -0.011095 \\
2 & 6 & 0 & 0.028438 & 0.347096 & -0.058087 \\
3 & 6 & 0 & -1.257578 & -0.513471 & -0.003279 \\
4 & 7 & 0 & -0.134805 & 1.620377 & -0.052824 \\
5 & 8 & 0 & -1.089230 & 2.387682 & -0.021781 \\
6 & 6 & 0 & -1.037987 & -2.022924 & -0.142933 \\
7 & 1 & 0 & -0.359259 & -2.433218 & 0.605554 \\
8 & 1 & 0 & -2.004224 & -2.517546 & -0.008373 \\
9 & 1 & 0 & -0.673245 & -2.293113 & -1.134782 \\
10 & 6 & 0 & -1.937548 & -0.242308 & 1.349026 \\
11 & 1 & 0 & -1.318205 & -0.597144 & 2.176996 \\
12 & 1 & 0 & -2.128065 & 0.823356 & 1.491386 \\
13 & 1 & 0 & -2.897116 & -0.765961 & 1.394760 \\
14 & 6 & 0 & 1.836811 & -1.086101 & -1.146821 \\
15 & 1 & 0 & 1.549236 & -0.675310 & -2.118431 \\
16 & 1 & 0 & 2.918197 & -1.250359 & -1.162575 \\
17 & 1 & 0 & 1.363706 & -2.059696 & -1.029836 \\
18 & 6 & 0 & 2.413399 & 1.108493 & -0.163752 \\
19 & 1 & 0 & 3.454906 & 0.779539 & -0.111299 \\
20 & 1 & 0 & 2.261229 & 1.607756 & -1.123457 \\
21 & 1 & 0 & 2.248482 & 1.843161 & 0.626986 \\
22 & 6 & 0 & -2.191580 & -0.090086 & -1.148288 \\
23 & 1 & 0 & -2.434507 & 0.972329 & -1.108690 \\
24 & 1 & 0 & -1.736445 & -0.303631 & -2.119238 \\
25 & 1 & 0 & -3.127250 & -0.653095 & -1.082858 \\
26 & 6 & 0 & 1.799248 & -0.754383 & 1.351005 \\
27 & 1 & 0 & 1.619171 & -0.045556 & 2.163322 \\
28 & 1 & 0 & 1.200142 & -1.645854 & 1.540791 \\
29 & 1 & 0 & 2.851696 & -1.051263 & 1.389096
\end{tabular}

Di-tert-butyl Iminyl - HLM

$\begin{array}{rrrrrr}1 & 6 & 0 & 1.384661 & -0.070233 & -0.000395 \\ 2 & 6 & 0 & 0.000011 & 0.640837 & 0.000098 \\ 3 & 6 & 0 & -1.384663 & -0.070255 & 0.000407 \\ 4 & 7 & 0 & -0.000056 & 1.889644 & 0.000055 \\ 5 & 6 & 0 & -1.454082 & -1.259069 & -0.962419 \\ 6 & 1 & 0 & -0.765372 & -2.063945 & -0.701661 \\ 7 & 1 & 0 & -2.465972 & -1.674796 & -0.933262 \\ 8 & 1 & 0 & -1.254500 & -0.952305 & -1.991895 \\ 9 & 6 & 0 & -1.708544 & -0.531239 & 1.430076 \\ 10 & 1 & 0 & -1.035525 & -1.311268 & 1.785718 \\ 11 & 1 & 0 & -1.661177 & 0.308670 & 2.127210 \\ 12 & 1 & 0 & -2.726081 & -0.933652 & 1.455112 \\ 13 & 6 & 0 & 1.708179 & -0.531743 & -1.430002 \\ 14 & 1 & 0 & 1.660337 & 0.307844 & -2.127505 \\ 15 & 1 & 0 & 2.725825 & -0.933865 & -1.455247 \\ 16 & 1 & 0 & 1.035273 & -1.312118 & -1.785133 \\ 17 & 6 & 0 & 2.455171 & 0.944820 & 0.422886 \\ 18 & 1 & 0 & 3.437992 & 0.464389 & 0.408781 \\ 19 & 1 & 0 & 2.479735 & 1.803265 & -0.250293 \\ 20 & 1 & 0 & 2.269106 & 1.319377 & 1.431793 \\ 21 & 6 & 0 & -2.455080 & 0.944614 & -0.423548\end{array}$




$\begin{array}{rrrrrr}22 & 1 & 0 & -2.479340 & 1.803671 & 0.248868 \\ 23 & 1 & 0 & -2.269261 & 1.318264 & -1.432834 \\ 24 & 1 & 0 & -3.437979 & 0.464366 & -0.408754 \\ 25 & 6 & 0 & 1.454398 & -1.258694 & 0.962923 \\ 26 & 1 & 0 & 1.255271 & -0.951539 & 1.992365 \\ 27 & 1 & 0 & 0.765513 & -2.063603 & 0.702730 \\ 28 & 1 & 0 & 2.466246 & -1.674490 & 0.933463\end{array}$

Di-tert-butyl Imine - MLM

$\begin{array}{cccrrr}1 & 6 & 0 & -1.379318 & -0.086326 & -0.000651 \\ 2 & 6 & 0 & -0.002636 & 0.648280 & -0.013130 \\ 3 & 6 & 0 & 1.372095 & -0.098328 & -0.005145 \\ 4 & 7 & 0 & -0.083009 & 1.924324 & -0.020434 \\ 5 & 1 & 0 & 0.839360 & 2.363058 & 0.017282 \\ 6 & 6 & 0 & 1.435854 & -1.271003 & 0.999781 \\ 7 & 1 & 0 & 0.706540 & -2.056272 & 0.793009 \\ 8 & 1 & 0 & 2.429796 & -1.732077 & 0.954073 \\ 9 & 1 & 0 & 1.280272 & -0.924112 & 2.027285 \\ 10 & 6 & 0 & 1.688599 & -0.616518 & -1.431746 \\ 11 & 1 & 0 & 1.009853 & -1.407595 & -1.755021 \\ 12 & 1 & 0 & 1.633580 & 0.197635 & -2.163400 \\ 13 & 1 & 0 & 2.707296 & -1.022781 & -1.457487 \\ 14 & 6 & 0 & -1.731121 & -0.462015 & 1.462670 \\ 15 & 1 & 0 & -1.696757 & 0.424815 & 2.104679 \\ 16 & 1 & 0 & -2.749694 & -0.867293 & 1.501864 \\ 17 & 1 & 0 & -1.061508 & -1.215957 & 1.884354 \\ 18 & 6 & 0 & -2.476471 & 0.879274 & -0.502910 \\ 19 & 1 & 0 & -3.445649 & 0.365497 & -0.490272 \\ 20 & 1 & 0 & -2.540339 & 1.772474 & 0.119965 \\ 21 & 1 & 0 & -2.274570 & 1.208960 & -1.527347 \\ 22 & 6 & 0 & 2.509739 & 0.874187 & 0.385147 \\ 23 & 1 & 0 & 2.665883 & 1.661324 & -0.361824 \\ 24 & 1 & 0 & 2.326191 & 1.348526 & 1.356044 \\ 25 & 1 & 0 & 3.451417 & 0.319618 & 0.458430 \\ 26 & 6 & 0 & -1.452122 & -1.348850 & -0.887970 \\ 27 & 1 & 0 & -1.259568 & -1.110804 & -1.939913 \\ 28 & 1 & 0 & -0.763952 & -2.142279 & -0.585618 \\ 29 & 1 & 0 & -2.464798 & -1.765218 & -0.829345\end{array}$

Di-tert-butyl Iminyl - MLM

$\begin{array}{cccrrr}1 & 6 & 0 & -1.397160 & -0.070762 & 0.001204 \\ 2 & 6 & 0 & -0.000045 & 0.642617 & -0.000019 \\ 3 & 6 & 0 & 1.397201 & -0.070786 & -0.001202 \\ 4 & 7 & 0 & 0.000024 & 1.898490 & -0.000034 \\ 5 & 6 & 0 & 1.471655 & -1.269639 & 0.965115 \\ 6 & 1 & 0 & 0.785120 & -2.078607 & 0.702154 \\ 7 & 1 & 0 & 2.486981 & -1.683750 & 0.938320 \\ 8 & 1 & 0 & 1.267736 & -0.966243 & 1.997500 \\ 9 & 6 & 0 & 1.728558 & -0.530796 & -1.440621 \\ 10 & 1 & 0 & 1.057648 & -1.314316 & -1.800443 \\ 11 & 1 & 0 & 1.678479 & 0.311440 & -2.138902 \\ 12 & 1 & 0 & 2.749667 & -0.931008 & -1.467251 \\ 13 & 6 & 0 & -1.728643 & -0.530463 & 1.440703 \\ 14 & 1 & 0 & -1.678631 & 0.311925 & 2.138807 \\ 15 & 1 & 0 & -2.749742 & -0.930692 & 1.467328 \\ 16 & 1 & 0 & -1.057734 & -1.313878 & 1.800783 \\ 17 & 6 & 0 & -2.472566 & 0.951202 & -0.431319 \\ 18 & 1 & 0 & -3.458681 & 0.471682 & -0.421486 \\ 19 & 1 & 0 & -2.501691 & 1.813182 & 0.240928 \\ 20 & 1 & 0 & -2.282663 & 1.324598 & -1.442746 \\ 21 & 6 & 0 & 2.472694 & 0.951193 & 0.431022 \\ 22 & 1 & 0 & 2.501831 & 1.813049 & -0.241388 \\ 23 & 1 & 0 & 2.282983 & 1.324822 & 1.442394 \\ 24 & 1 & 0 & 3.458779 & 0.471602 & 0.421155 \\ 25 & 6 & 0 & -1.471717 & -1.269869 & -0.964874 \\ 26 & 1 & 0 & -1.267636 & -0.966778 & -1.997309 \\ 27 & 1 & 0 & -0.785352 & -2.078886 & -0.701622 \\ 28 & 1 & 0 & -2.487122 & -1.683760 & -0.938047\end{array}$


Tert-butyl 1-Adamantyl Ketone Oxime Ether - HLM

\begin{tabular}{|c|c|c|c|c|c|}
\hline 1 & 6 & 0 & -3.994956 & -1.777732 & 1.156190 \\
\hline 2 & 1 & 0 & -3.419919 & -2.263819 & 1.952910 \\
\hline 3 & 1 & 0 & -5.016779 & -2.171923 & 1.209231 \\
\hline 4 & 6 & 0 & -3.372787 & -2.090376 & -0.207452 \\
\hline 5 & 1 & 0 & -3.333793 & -3.175699 & -0.352954 \\
\hline 6 & 6 & 0 & -4.001178 & -0.261690 & 1.364094 \\
\hline 7 & 1 & 0 & -4.436695 & -0.022491 & 2.340678 \\
\hline 8 & 6 & 0 & -2.561631 & 0.268913 & 1.322601 \\
\hline 9 & 1 & 0 & -1.960121 & -0.220764 & 2.097245 \\
\hline 10 & 1 & 0 & -2.565572 & 1.336280 & 1.553298 \\
\hline 11 & 6 & 0 & -1.943204 & -1.541652 & -0.257844 \\
\hline 12 & 1 & 0 & -1.477068 & -1.790564 & -1.215687 \\
\hline 13 & 1 & 0 & -1.334003 & -2.022521 & 0.510528 \\
\hline 14 & 6 & 0 & -1.913783 & -0.008016 & -0.056508 \\
\hline 15 & 6 & 0 & -2.786145 & 0.605605 & -1.175848 \\
\hline 16 & 1 & 0 & -2.825369 & 1.690587 & -1.095547 \\
\hline 17 & 1 & 0 & -2.337136 & 0.368725 & -2.147712 \\
\hline 18 & 6 & 0 & -4.214983 & -1.451672 & -1.313537 \\
\hline 19 & 1 & 0 & -5.238781 & -1.843618 & -1.290843 \\
\hline 20 & 1 & 0 & -3.796914 & -1.698600 & -2.296464 \\
\hline 21 & 6 & 0 & -4.827866 & 0.400280 & 0.255165 \\
\hline 22 & 1 & 0 & -5.865073 & 0.048222 & 0.302179 \\
\hline 23 & 1 & 0 & -4.853015 & 1.487086 & 0.399394 \\
\hline 24 & 6 & 0 & -4.220592 & 0.063842 & -1.112221 \\
\hline 25 & 1 & 0 & -4.810451 & 0.541825 & -1.902303 \\
\hline 26 & 6 & 0 & -0.463287 & 0.518166 & -0.076533 \\
\hline 27 & 6 & 0 & -0.033061 & 2.001309 & 0.044110 \\
\hline 28 & 6 & 0 & 0.684763 & 2.171037 & 1.397957 \\
\hline 29 & 1 & 0 & 1.073956 & 3.190863 & 1.477419 \\
\hline 30 & 1 & 0 & 0336 & 2.012544 & 2.227474 \\
\hline 31 & 1 & 0 & 1.516499 & 1.476795 & 1.499381 \\
\hline 32 & 6 & 0 & -1.146256 & 3.059921 & -0.007452 \\
\hline 33 & 1 & 0 & -1.955293 & 2.895408 & 0.703517 \\
\hline 34 & 1 & 0 & -0.692184 & 4.023329 & 0.242764 \\
\hline 35 & 1 & 0 & -1.573586 & 3.164214 & -1.005851 \\
\hline 36 & 6 & 0 & 0.918335 & 2.364848 & -1.114199 \\
\hline 37 & 1 & 0 & 192 & 3.423346 & -1.034833 \\
\hline 38 & 1 & 0 & 1.829898 & 1.775048 & -1.102195 \\
\hline 39 & 1 & 0 & 0.420455 & 2.219830 & -2.077429 \\
\hline 40 & 7 & 0 & 0.374652 & -0.451556 & -0.130954 \\
\hline 41 & 8 & 0 & 1.721524 & -0.118771 & -0.073549 \\
\hline 42 & 6 & 0 & 2.479479 & -1.328699 & -0.156124 \\
\hline 43 & 1 & 0 & 2.249327 & -1.830306 & -1.101424 \\
\hline 44 & 1 & 0 & 986 & 9679 & 5687 \\
\hline 45 & 6 & 0 & 3.931597 & -0.970187 & -0.071765 \\
\hline 46 & 6 & 0 & 4.581748 & -0.935360 & 1.160581 \\
\hline 47 & 6 & 0 & 4.648404 & -0.640204 & -1.221009 \\
\hline 48 & 6 & 0 & 5.922806 & -0.582691 & 1.244616 \\
\hline 49 & 1 & 0 & 4.030422 & -1.190256 & 2.060076 \\
\hline 50 & 6 & 0 & 5.989345 & -0.286690 & -1.141946 \\
\hline 51 & 1 & 0 & 4.149369 & -0.664529 & -2.184492 \\
\hline 52 & 6 & 0 & 6.628857 & -0.257809 & 0.092210 \\
\hline 53 & 1 & 0 & 6.418032 & -0.563531 & 2.209069 \\
\hline 54 & 1 & 0 & 6.537042 & -0.036313 & -2.043705 \\
\hline 55 & 1 & 0 & 7.676520 & 0.014705 & 0.155141 \\
\hline
\end{tabular}

Tert-butyl 1-Adamantyl Ketone Oxime Ether Z-isomer - HLM

$\begin{array}{cccccc}1 & 6 & 0 & 2.583662 & -2.611090 & 1.309595 \\ 2 & 1 & 0 & 3.159474 & -2.150153 & 2.121105 \\ 3 & 1 & 0 & 2.712772 & -3.696091 & 1.401193 \\ 4 & 6 & 0 & 3.103529 & -2.133445 & -0.047731 \\ 5 & 1 & 0 & 4.169519 & -2.370696 & -0.138991 \\ 6 & 6 & 0 & 1.106086 & -2.235283 & 1.434544 \\ 7 & 1 & 0 & 0.718825 & -2.567895 & 2.404151 \\ 8 & 6 & 0 & 0.958553 & -0.713215 & 1.342874 \\ 9 & 1 & 0 & 1.540110 & -0.235887 & 2.140863 \\ 10 & 1 & 0 & -0.084716 & -0.429687 & 1.482940 \\ 11 & 6 & 0 & 2.937796 & -0.611491 & -0.178812 \\ 12 & 1 & 0 & 3.309448 & -0.318079 & -1.162937\end{array}$




$\begin{array}{rrrrrr}13 & 1 & 0 & 3.568211 & -0.124161 & 0.568475 \\ 14 & 6 & 0 & 1.458734 & -0.187484 & -0.029469 \\ 15 & 6 & 0 & 0.699851 & -0.914155 & -1.172823 \\ 16 & 1 & 0 & -0.354581 & -0.647659 & -1.152626 \\ 17 & 1 & 0 & 1.105871 & -0.576337 & -2.134243 \\ 18 & 6 & 0 & 2.323621 & -2.826960 & -1.166416 \\ 19 & 1 & 0 & 2.438782 & -3.914877 & -1.092410 \\ 20 & 1 & 0 & 2.718603 & -2.527308 & -2.144448 \\ 21 & 6 & 0 & 0.306161 & -2.898612 & 0.307233 \\ 22 & 1 & 0 & 0.380205 & -3.989789 & 0.387039 \\ 23 & 1 & 0 & -0.754279 & -2.639394 & 0.396928 \\ 24 & 6 & 0 & 0.850123 & -2.435894 & -1.050104 \\ 25 & 1 & 0 & 0.275779 & -2.908737 & -1.854694 \\ 26 & 6 & 0 & 1.181994 & 1.330121 & -0.088552 \\ 27 & 6 & 0 & 2.260843 & 2.442902 & -0.102679 \\ 28 & 6 & 0 & 2.982471 & 2.472356 & 1.256331 \\ 29 & 1 & 0 & 3.737630 & 3.264410 & 1.252137 \\ 30 & 1 & 0 & 2.272975 & 2.688381 & 2.059299 \\ 31 & 1 & 0 & 3.489223 & 1.537112 & 1.495295 \\ 32 & 6 & 0 & 1.614429 & 3.825074 & -0.289373 \\ 33 & 1 & 0 & 0.902922 & 4.054068 & 0.502717 \\ 34 & 1 & 0 & 2.405270 & 4.581360 & -0.279872 \\ 35 & 1 & 0 & 1.082514 & 3.892793 & -1.240227 \\ 36 & 6 & 0 & 3.279924 & 2.282402 & -1.245720 \\ 37 & 1 & 0 & 3.822455 & 3.223844 & -1.369746 \\ 38 & 1 & 0 & 4.021059 & 1.508730 & -1.057603 \\ 39 & 1 & 0 & 2.782600 & 2.064114 & -2.195041 \\ 40 & 7 & 0 & 0.007312 & 1.844045 & -0.052360 \\ 41 & 8 & 0 & -1.063969 & 0.963002 & 0.037336 \\ 42 & 6 & 0 & -2.257088 & 1.733251 & 0.174947 \\ 43 & 1 & 0 & -2.259972 & 2.237941 & 1.146858 \\ 44 & 1 & 0 & -2.266356 & 2.501132 & -0.606195 \\ 45 & 6 & 0 & -3.438832 & 0.818938 & 0.048103 \\ 46 & 6 & 0 & -4.403915 & 0.765665 & 1.050555 \\ 47 & 6 & 0 & -5.517636 & -0.056821 & 0.921221 \\ 48 & 6 & 0 & -5.670115 & -0.846053 & -0.211243 \\ 49 & 6 & 0 & -4.706349 & -0.806409 & -1.214173 \\ 50 & 6 & 0 & -3.600714 & 0.022631 & -1.086380 \\ 51 & 1 & 0 & -4.282169 & 1.374006 & 1.941303 \\ 52 & 1 & 0 & -6.262285 & -0.085077 & 1.708998 \\ 53 & 1 & 0 & -6.535709 & -1.491091 & -0.313178 \\ 54 & 1 & 0 & -4.820163 & -1.421179 & -2.100272 \\ 55 & 1 & 0 & -2.852217 & 0.055297 & -1.870564\end{array}$

Tert-butyl 1-Adamantyl Ketone Iminoxyl - HLM

$\begin{array}{cccccc}1 & 6 & 0 & 2.853703 & 0.685570 & 1.209073 \\ 2 & 1 & 0 & 2.568806 & 1.332109 & 2.047178 \\ 3 & 1 & 0 & 3.941061 & 0.555688 & 1.258906 \\ 4 & 6 & 0 & 2.459497 & 1.339283 & -0.119120 \\ 5 & 1 & 0 & 2.936682 & 2.321819 & -0.201520 \\ 6 & 6 & 0 & 2.152863 & -0.669902 & 1.327871 \\ 7 & 1 & 0 & 2.421258 & -1.143700 & 2.278470 \\ 8 & 6 & 0 & 0.632935 & -0.466667 & 1.288035 \\ 9 & 1 & 0 & 0.319887 & 0.186602 & 2.110512 \\ 10 & 1 & 0 & 0.136272 & -1.428602 & 1.439439 \\ 11 & 6 & 0 & 0.940181 & 1.527073 & -0.168128 \\ 12 & 1 & 0 & 0.652981 & 2.019723 & -1.103243 \\ 13 & 1 & 0 & 0.622347 & 2.187387 & 0.645201 \\ 14 & 6 & 0 & 0.200626 & 0.174764 & -0.053365 \\ 15 & 6 & 0 & 0.684056 & -0.711917 & -1.222877 \\ 16 & 1 & 0 & 0.204410 & -1.690519 & -1.190179 \\ 17 & 1 & 0 & 0.396957 & -0.242831 & -2.171438 \\ 18 & 6 & 0 & 2.906593 & 0.448518 & -1.281092 \\ 19 & 1 & 0 & 3.994504 & 0.315508 & -1.260659 \\ 20 & 1 & 0 & 2.658156 & 0.922303 & -2.237802 \\ 21 & 6 & 0 & 2.582565 & -1.569760 & 0.163541 \\ 22 & 1 & 0 & 3.664289 & -1.742100 & 0.203117 \\ 23 & 1 & 0 & 2.100195 & -2.550994 & 0.246077 \\ 24 & 6 & 0 & 2.204746 & -0.906241 & -1.166127 \\ 25 & 1 & 0 & 2.507679 & -1.552051 & -1.997565\end{array}$




$\begin{array}{rrrrrr}26 & 6 & 0 & -1.309156 & 0.408597 & -0.075314 \\ 27 & 6 & 0 & -2.458476 & -0.628749 & 0.000368 \\ 28 & 6 & 0 & -3.130139 & -0.467898 & 1.374753 \\ 29 & 1 & 0 & -4.008690 & -1.117169 & 1.438493 \\ 30 & 1 & 0 & -2.445100 & -0.744798 & 2.180497 \\ 31 & 1 & 0 & -3.459365 & 0.560461 & 1.537785 \\ 32 & 6 & 0 & -2.041777 & -2.094223 & -0.166192 \\ 33 & 1 & 0 & -1.274477 & -2.412406 & 0.539807 \\ 34 & 1 & 0 & -2.923879 & -2.715697 & 0.014161 \\ 35 & 1 & 0 & -1.698755 & -2.311175 & -1.179082 \\ 36 & 6 & 0 & -3.477452 & -0.331045 & -1.111690 \\ 37 & 1 & 0 & -4.312645 & -1.033591 & -1.036988 \\ 38 & 1 & 0 & -3.880816 & 0.679541 & -1.041335 \\ 39 & 1 & 0 & -3.020754 & -0.452393 & -2.097671 \\ 40 & 7 & 0 & -1.657693 & 1.644922 & -0.057887 \\ 41 & 8 & 0 & -2.712716 & 2.264751 & -0.001922\end{array}$

Tert-butyl 1-Adamantyl Ketone Iminoxyl Z-isomer - HLM

$\begin{array}{rrrrrr}1 & 6 & 0 & 2.977632 & -0.169867 & 0.327638 \\ 2 & 1 & 0 & 3.008586 & -0.179086 & 1.423636 \\ 3 & 1 & 0 & 4.016037 & -0.201541 & -0.022031 \\ 4 & 6 & 0 & 2.295928 & 1.106814 & -0.172067 \\ 5 & 1 & 0 & 2.832064 & 1.983672 & 0.207467 \\ 6 & 6 & 0 & 2.205563 & -1.387771 & -0.187060 \\ 7 & 1 & 0 & 2.682551 & -2.307471 & 0.168823 \\ 8 & 6 & 0 & 0.770346 & -1.333867 & 0.345847 \\ 9 & 1 & 0 & 0.777741 & -1.347746 & 1.441962 \\ 10 & 1 & 0 & 0.214670 & -2.215826 & 0.013050 \\ 11 & 6 & 0 & 0.847374 & 1.169860 & 0.338526 \\ 12 & 1 & 0 & 0.386611 & 2.087426 & -0.036776 \\ 13 & 1 & 0 & 0.856926 & 1.228400 & 1.430075 \\ 14 & 6 & 0 & 0.047796 & -0.055924 & -0.144092 \\ 15 & 6 & 0 & 0.074929 & -0.025845 & -1.690566 \\ 16 & 1 & 0 & -0.497525 & -0.864980 & -2.093582 \\ 17 & 1 & 0 & -0.411400 & 0.893517 & -2.037938 \\ 18 & 6 & 0 & 2.299489 & 1.122667 & -1.702448 \\ 19 & 1 & 0 & 3.328907 & 1.096166 & -2.078410 \\ 20 & 1 & 0 & 1.846909 & 2.050167 & -2.072422 \\ 21 & 6 & 0 & 2.185186 & -1.376144 & -1.719413 \\ 22 & 1 & 0 & 3.207692 & -1.440586 & -2.109389 \\ 23 & 1 & 0 & 1.641973 & -2.250579 & -2.094610 \\ 24 & 6 & 0 & 1.515648 & -0.088361 & -2.212947 \\ 25 & 1 & 0 & 1.493344 & -0.082103 & -3.308053 \\ 26 & 6 & 0 & -1.413698 & -0.125221 & 0.347195 \\ 27 & 6 & 0 & -2.108168 & 0.721852 & 1.421986 \\ 28 & 6 & 0 & -1.478119 & 0.446094 & 2.798018 \\ 29 & 1 & 0 & -1.986461 & 1.041177 & 3.562945 \\ 30 & 1 & 0 & -1.582937 & -0.608470 & 3.065840 \\ 31 & 1 & 0 & -0.418056 & 0.700765 & 2.834792 \\ 32 & 6 & 0 & -3.589696 & 0.328618 & 1.506264 \\ 33 & 1 & 0 & -3.715983 & -0.727368 & 1.752947 \\ 34 & 1 & 0 & -4.073605 & 0.921366 & 2.287469 \\ 35 & 1 & 0 & -4.107687 & 0.518577 & 0.563365 \\ 36 & 6 & 0 & -2.057570 & 2.227698 & 1.112618 \\ 37 & 1 & 0 & -2.742851 & 2.754146 & 1.783322 \\ 38 & 1 & 0 & -1.068774 & 2.660185 & 1.257818 \\ 39 & 1 & 0 & -2.376894 & 2.430484 & 0.086787 \\ 40 & 7 & 0 & -2.135532 & -1.076103 & -0.126438 \\ 41 & 8 & 0 & -1.974870 & -1.996399 & -0.919876\end{array}$

Tert-butyl 1-Adamantyl Ketone Iminyl - HLM

$\begin{array}{rrrrrr}1 & 6 & 0 & -2.255539 & -1.562081 & 0.577005 \\ 2 & 1 & 0 & -1.687952 & -2.416166 & 0.965180 \\ 3 & 1 & 0 & -3.315253 & -1.839290 & 0.613127 \\ 4 & 6 & 0 & -1.840979 & -1.265127 & -0.867877 \\ 5 & 1 & 0 & -2.011536 & -2.150901 & -1.489506 \\ 6 & 6 & 0 & -2.005101 & -0.323417 & 1.443312 \\ 7 & 1 & 0 & -2.295341 & -0.532350 & 2.478810 \\ 8 & 6 & 0 & -0.511913 & 0.027533 & 1.411981 \\ 9 & 1 & 0 & 0.064767 & -0.796405 & 1.839823\end{array}$




$\begin{array}{rrrrrr}10 & 1 & 0 & -0.317304 & 0.911818 & 2.028059 \\ 11 & 6 & 0 & -0.345831 & -0.915678 & -0.914114 \\ 12 & 1 & 0 & -0.049722 & -0.710717 & -1.948712 \\ 13 & 1 & 0 & 0.230753 & -1.781006 & -0.576634 \\ 14 & 6 & 0 & -0.055229 & 0.316677 & -0.036959 \\ 15 & 6 & 0 & -0.907866 & 1.488495 & -0.572733 \\ 16 & 1 & 0 & -0.712834 & 2.381693 & 0.027486 \\ 17 & 1 & 0 & -0.594248 & 1.724619 & -1.595169 \\ 18 & 6 & 0 & -2.662291 & -0.090640 & -1.406019 \\ 19 & 1 & 0 & -3.729430 & -0.341650 & -1.397396 \\ 20 & 1 & 0 & -2.389361 & 0.114379 & -2.447662 \\ 21 & 6 & 0 & -2.822852 & 0.853622 & 0.904147 \\ 22 & 1 & 0 & -3.892984 & 0.619615 & 0.945576 \\ 23 & 1 & 0 & -2.662589 & 1.739963 & 1.528459 \\ 24 & 6 & 0 & -2.399293 & 1.143439 & -0.539143 \\ 25 & 1 & 0 & -2.968814 & 1.995261 & -0.926044 \\ 26 & 6 & 0 & 1.433547 & 0.753544 & -0.012519 \\ 27 & 6 & 0 & 2.664554 & -0.198991 & 0.003631 \\ 28 & 6 & 0 & 2.521925 & -1.334872 & 1.021780 \\ 29 & 1 & 0 & 3.433104 & -1.940987 & 1.004789 \\ 30 & 1 & 0 & 2.403188 & -0.947001 & 2.036424 \\ 31 & 1 & 0 & 1.683969 & -1.999051 & 0.806222 \\ 32 & 6 & 0 & 3.910524 & 0.616111 & 0.375440 \\ 33 & 1 & 0 & 3.809863 & 1.065325 & 1.365559 \\ 34 & 1 & 0 & 4.785551 & -0.040610 & 0.380622 \\ 35 & 1 & 0 & 4.086923 & 1.422177 & -0.338509 \\ 36 & 6 & 0 & 2.878692 & -0.773364 & -1.405310 \\ 37 & 1 & 0 & 3.823261 & -1.325666 & -1.426944 \\ 38 & 1 & 0 & 2.087335 & -1.458844 & -1.707927 \\ 39 & 1 & 0 & 2.941908 & 0.028426 & -2.144917 \\ 40 & 7 & 0 & 1.666093 & 1.980836 & 0.011937\end{array}$

Tert-butyl 1-Adamantyl Ketone Imine - MLM

$\begin{array}{cccccc}1 & 6 & 0 & -2.696518 & -.155423 & -1.411021 \\ 2 & 1 & 0 & -2.425024 & .005157 & -2.463701 \\ 3 & 1 & 0 & -3.766381 & -.406746 & -1.391108 \\ 4 & 6 & 0 & -2.426918 & 1.119857 & -.591564 \\ 5 & 1 & 0 & -2.995649 & 1.958959 & -1.014523 \\ 6 & 6 & 0 & -1.867508 & -1.310431 & -.821200 \\ 7 & 1 & 0 & -2.036193 & -2.224964 & -1.405882 \\ 8 & 6 & 0 & -.364250 & -.958006 & -.882964 \\ 9 & 1 & 0 & -.075522 & -.792752 & -1.929319 \\ 10 & 1 & 0 & .207103 & -1.820930 & -.526695 \\ 11 & 6 & 0 & -.925754 & 1.466024 & -.636202 \\ 12 & 1 & 0 & -.731719 & 2.385801 & -.080165 \\ 13 & 1 & 0 & -.612599 & 1.661932 & -1.670464 \\ 14 & 6 & 0 & -.054592 & .316245 & -.048482 \\ 15 & 6 & 0 & -.531909 & .083789 & 1.420447 \\ 16 & 1 & 0 & .040989 & -.720914 & 1.894120 \\ 17 & 1 & 0 & -.341106 & .996489 & 1.999317 \\ 18 & 6 & 0 & -2.856986 & .889805 & .870463 \\ 19 & 1 & 0 & -3.929961 & .656901 & .920765 \\ 20 & 1 & 0 & -2.699193 & 1.804262 & 1.458519 \\ 21 & 6 & 0 & -2.283847 & -1.550459 & .643778 \\ 22 & 1 & 0 & -3.345344 & -1.830030 & .692476 \\ 23 & 1 & 0 & -1.713766 & -2.389539 & 1.067651 \\ 24 & 6 & 0 & -2.033700 & -.269087 & 1.464078 \\ 25 & 1 & 0 & -2.327624 & -.436169 & 2.509304 \\ 26 & 6 & 0 & 1.438552 & .761817 & -.021244 \\ 27 & 6 & 0 & 2.642340 & -.238468 & -.000799 \\ 28 & 6 & 0 & 2.846615 & -.847867 & -1.412116 \\ 29 & 1 & 0 & 3.780108 & -1.423720 & -1.431084 \\ 30 & 1 & 0 & 2.039519 & -1.520588 & -1.705890 \\ 31 & 1 & 0 & 2.924498 & -.059588 & -2.169699 \\ 32 & 6 & 0 & 2.477617 & -1.369402 & 1.040993 \\ 33 & 1 & 0 & 1.603943 & -1.998036 & .860599 \\ 34 & 1 & 0 & 3.358877 & -2.021857 & 1.011230 \\ 35 & 1 & 0 & 2.400771 & -.964769 & 2.056390 \\ 36 & 6 & 0 & 3.951541 & .501060 & .360049 \\ 37 & 1 & 0 & 4.769052 & -.224633 & .428852\end{array}$




$\begin{array}{llllll}38 & 1 & 0 & 4.242382 & 1.233931 & -.401711 \\ 39 & 1 & 0 & 3.880671 & 1.013016 & 1.326711 \\ 40 & 7 & 0 & 1.609455 & 2.029071 & .006637 \\ 41 & 1 & 0 & 2.597882 & 2.278576 & .072566\end{array}$

Tert-butyl 1-Adamantyl Ketone Imine Z-isomer - MLM

$\begin{array}{rrrrrr}1 & 6 & 0 & 2.759854 & -0.120082 & 0.505077 \\ 2 & 1 & 0 & 2.653696 & 0.045357 & 1.586343 \\ 3 & 1 & 0 & 3.836486 & -0.194701 & 0.299065 \\ 4 & 6 & 0 & 2.152286 & 1.063099 & -0.276022 \\ 5 & 1 & 0 & 2.652337 & 1.995223 & 0.019248 \\ 6 & 6 & 0 & 2.050170 & -1.426590 & 0.095051 \\ 7 & 1 & 0 & 2.480664 & -2.270349 & 0.650749 \\ 8 & 6 & 0 & 0.547702 & -1.324038 & 0.436793 \\ 9 & 1 & 0 & 0.434897 & -1.221340 & 1.518725 \\ 10 & 1 & 0 & 0.031584 & -2.250747 & 0.150734 \\ 11 & 6 & 0 & 0.648999 & 1.183504 & 0.053248 \\ 12 & 1 & 0 & 0.216483 & 2.025777 & -0.503322 \\ 13 & 1 & 0 & 0.538450 & 1.417551 & 1.115460 \\ 14 & 6 & 0 & -0.114296 & -0.123420 & -0.310141 \\ 15 & 6 & 0 & 0.112147 & -0.366855 & -1.831304 \\ 16 & 1 & 0 & -0.370333 & -1.303929 & -2.140806 \\ 17 & 1 & 0 & -0.352375 & 0.443652 & -2.409512 \\ 18 & 6 & 0 & 2.331731 & 0.833098 & -1.786905 \\ 19 & 1 & 0 & 3.398745 & 0.768260 & -2.040496 \\ 20 & 1 & 0 & 1.919543 & 1.681777 & -2.350171 \\ 21 & 6 & 0 & 2.231016 & -1.654129 & -1.416626 \\ 22 & 1 & 0 & 3.297621 & -1.748163 & -1.662892 \\ 23 & 1 & 0 & 1.748895 & -2.594192 & -1.718748 \\ 24 & 6 & 0 & 1.611195 & -0.468627 & -2.181699 \\ 25 & 1 & 0 & 1.708263 & -0.633785 & -3.262973 \\ 26 & 6 & 0 & -1.639601 & -0.052654 & 0.014116 \\ 27 & 6 & 0 & -2.215751 & 0.487622 & 1.363887 \\ 28 & 6 & 0 & -1.411406 & 0.126185 & 2.631941 \\ 29 & 1 & 0 & -1.921776 & 0.550611 & 3.504788 \\ 30 & 1 & 0 & -1.362813 & -0.958037 & 2.781743 \\ 31 & 1 & 0 & -0.391287 & 0.518380 & 2.638205 \\ 32 & 6 & 0 & -3.640814 & -0.076828 & 1.567699 \\ 33 & 1 & 0 & -3.628448 & -1.170901 & 1.618106 \\ 34 & 1 & 0 & -4.052071 & 0.306142 & 2.509887 \\ 35 & 1 & 0 & -4.304702 & 0.204663 & 0.749625 \\ 36 & 6 & 0 & -2.341079 & 2.030419 & 1.259144 \\ 37 & 1 & 0 & -2.861381 & 2.417787 & 2.143872 \\ 38 & 1 & 0 & -1.372315 & 2.533900 & 1.200709 \\ 39 & 1 & 0 & -2.925310 & 2.307777 & 0.375355 \\ 40 & 7 & 0 & -2.534636 & -0.405037 & -0.828869 \\ 41 & 1 & 0 & -2.125325 & -0.699682 & -1.717167\end{array}$

Tert-butyl 1-Adamantyl Ketone Iminyl - MLM

$\begin{array}{rrrrrr}1 & 6 & 0 & -2.279232 & -1.570490 & 0.579856 \\ 2 & 1 & 0 & -1.713439 & -2.428756 & 0.968895 \\ 3 & 1 & 0 & -3.341947 & -1.846662 & 0.615690 \\ 4 & 6 & 0 & -1.860127 & -1.271988 & -0.873670 \\ 5 & 1 & 0 & -2.031972 & -2.159638 & -1.497041 \\ 6 & 6 & 0 & -2.024621 & -0.325262 & 1.452732 \\ 7 & 1 & 0 & -2.317109 & -0.534641 & 2.490370 \\ 8 & 6 & 0 & -0.520802 & 0.024406 & 1.422632 \\ 9 & 1 & 0 & 0.053640 & -0.803140 & 1.852607 \\ 10 & 1 & 0 & -0.324639 & 0.907571 & 2.043791 \\ 11 & 6 & 0 & -0.354397 & -0.923872 & -0.918110 \\ 12 & 1 & 0 & -0.055518 & -0.722628 & -1.954962 \\ 13 & 1 & 0 & 0.218746 & -1.793893 & -0.580782 \\ 14 & 6 & 0 & -0.056325 & 0.316336 & -0.035250 \\ 15 & 6 & 0 & -0.915145 & 1.496511 & -0.575853 \\ 16 & 1 & 0 & -0.721630 & 2.393223 & 0.023119 \\ 17 & 1 & 0 & -0.602223 & 1.733381 & -1.600865 \\ 18 & 6 & 0 & -2.683816 & -0.088448 & -1.415384 \\ 19 & 1 & 0 & -3.754054 & -0.337694 & -1.408462 \\ 20 & 1 & 0 & -2.410039 & 0.116492 & -2.459599 \\ 21 & 6 & 0 & -2.844506 & 0.861074 & 0.909691\end{array}$




\begin{tabular}{|c|c|c|c|c|c|}
\hline & & & & & \\
\hline 22 & 1 & 0 & -3.917815 & 0.629312 & 0.950894 \\
\hline 23 & 1 & 0 & -2.684004 & 1.749088 & 1.536247 \\
\hline 24 & 6 & 0 & -2.416949 & 1.152385 & -0.542437 \\
\hline 25 & 1 & 0 & -2.985758 & 2.007775 & -0.930261 \\
\hline 26 & 6 & 0 & 1.445211 & 0.755790 & -0.012306 \\
\hline 27 & 6 & 0 & 2.689250 & -0.199724 & 0.000816 \\
\hline 28 & 6 & 0 & 2.552765 & -1.342467 & 1.027783 \\
\hline 29 & 1 & 0 & 3.469938 & -1.944265 & 1.016475 \\
\hline 30 & 1 & 0 & 2.426533 & -0.952999 & 2.043791 \\
\hline 31 & 1 & 0 & 1.718983 & -2.015619 & 0.812305 \\
\hline 32 & 6 & 0 & 3.941867 & 0.624532 & 0.374870 \\
\hline 33 & 1 & 0 & 3.841947 & 1.073647 & 1.368005 \\
\hline 34 & 1 & 0 & 4.821586 & -0.030179 & 0.379748 \\
\hline 35 & 1 & 0 & 4.118587 & 1.433536 & -0.339434 \\
\hline 36 & 6 & 0 & 2.910020 & -0.779810 & -1.416196 \\
\hline 37 & 1 & 0 & 3.853677 & -1.338929 & -1.435149 \\
\hline 38 & 1 & 0 & 2.115649 & -1.463149 & -1.724382 \\
\hline 39 & 1 & 0 & 2.980680 & 0.022295 & -2.158853 \\
\hline 40 & 7 & 0 & 1.677289 & 1.990290 & 0.016117 \\
\hline Benz & HLI & & & & \\
\hline 1 & 6 & 0 & -1.211798 & -0.000005 & -0.256505 \\
\hline 2 & 6 & 0 & -1.211666 & -0.000010 & 1.123393 \\
\hline 3 & 6 & 0 & -0.008097 & -0.000007 & 1.831508 \\
\hline 4 & 6 & 0 & 1.201686 & 0.000001 & 1.134062 \\
\hline 5 & 6 & 0 & 1.214018 & 0.000007 & -0.245780 \\
\hline 6 & 6 & 0 & 0.004379 & 0.000004 & -0.990355 \\
\hline 7 & 1 & 0 & -2.150077 & -0.000008 & -0.801409 \\
\hline 8 & 1 & 0 & -2.153471 & -0.000016 & 1.661328 \\
\hline 9 & 1 & 0 & -0.012889 & -0.000011 & 2.915275 \\
\hline 10 & 1 & 0 & 2.138697 & 0.000004 & 1.680304 \\
\hline 11 & 1 & 0 & 2.157079 & 0.000013 & -0.782366 \\
\hline 12 & 6 & 0 & 0.010574 & 0.000009 & -2.391722 \\
\hline 13 & 1 & 0 & 0.940736 & 0.000023 & -2.946271 \\
\hline 14 & 1 & 0 & -0.914648 & -0.000002 & -2.954474 \\
\hline Benz & $y \mid$ & & & & \\
\hline 1 & 8 & 0 & 1.044057 & 1.608534 & -2.119718 \\
\hline 2 & 6 & 0 & 1.025605 & 1.580448 & -0.773019 \\
\hline 3 & 1 & 0 & 2.078703 & 1.623125 & -0.420663 \\
\hline 4 & 1 & 0 & 0.635730 & 2.559643 & -0.420694 \\
\hline 5 & 6 & 0 & 0.288769 & 0.445053 & -0.103956 \\
\hline 6 & 6 & 0 & 0.237705 & 0.367659 & 1.286768 \\
\hline 7 & 6 & 0 & -0.438464 & -0.674316 & 1.908179 \\
\hline 8 & 6 & 0 & -1.069623 & -1.648215 & 1.141892 \\
\hline 9 & 6 & 0 & -1.020219 & -1.573359 & -0.245462 \\
\hline 10 & 6 & 0 & -0.343485 & -0.530522 & -0.866522 \\
\hline 11 & 1 & 0 & 0.729481 & 1.126515 & 1.889505 \\
\hline 12 & 1 & 0 & -0.473303 & -0.726981 & 2.990748 \\
\hline 13 & 1 & 0 & -1.597878 & -2.462248 & 1.625479 \\
\hline 14 & 1 & 0 & -1.510978 & -2.330604 & -0.847038 \\
\hline 15 & 1 & 0 & -0.295936 & -0.458202 & -1.946873 \\
\hline
\end{tabular}

Table S3. Calculated minimum energy geometries in acetone oxime and its iminoxyl radical from Table 4.

\begin{tabular}{cccrrr}
\multicolumn{7}{c}{ Parent } & - G 3 & or & GMP2 & & & \\
1 & 6 & 0 & 1.769326 & -0.621341 & 0.000001 \\
2 & 1 & 0 & 2.330790 & -0.296730 & 0.882454 \\
3 & 1 & 0 & 1.704257 & -1.710750 & 0.000060 \\
4 & 1 & 0 & 2.330751 & -0.296824 & -0.882513 \\
5 & 6 & 0 & 0.397216 & -0.023112 & -0.000003 \\
6 & 6 & 0 & 0.216174 & 1.464841 & -0.000004 \\
7 & 1 & 0 & -0.358473 & 1.775741 & -0.877186 \\
8 & 1 & 0 & -0.358116 & 1.775767 & 0.877408 \\
9 & 1 & 0 & 1.180130 & 1.976739 & -0.000200
\end{tabular}




\begin{tabular}{|c|c|c|c|c|c|}
\hline 10 & 7 & 0 & -0.586280 & -0.857518 & 0.000000 \\
\hline 11 & 8 & 0 & -1.820613 & -0.156356 & 0.000003 \\
\hline 12 & 1 & 0 & -2.456766 & -0.892793 & -0.000022 \\
\hline \multicolumn{6}{|c|}{ Radical - G3 or G3MP2 } \\
\hline 1 & 6 & 0 & -1.558864 & -0.877970 & -0.000001 \\
\hline 2 & 1 & 0 & -2.170026 & -0.666321 & -0.882615 \\
\hline 3 & 1 & 0 & -1.288894 & -1.934840 & -0.000006 \\
\hline 4 & 1 & 0 & -2.170022 & -0.666329 & 0.882619 \\
\hline 5 & 6 & 0 & -0.332372 & -0.018485 & 0.000000 \\
\hline 6 & 6 & 0 & -0.469343 & 1.478433 & 0.000000 \\
\hline 7 & 1 & 0 & -1.026267 & 1.804465 & 0.883461 \\
\hline 8 & 1 & 0 & 0.514091 & 1.948920 & -0.000004 \\
\hline 9 & 1 & 0 & -1.026272 & 1.804463 & -0.883458 \\
\hline 10 & 7 & 0 & 0.788418 & -0.560250 & 0.000003 \\
\hline 11 & 8 & 0 & 1.976493 & -0.232560 & -0.000002 \\
\hline \multicolumn{6}{|c|}{ Parent - CBS-QB3 } \\
\hline 1 & 6 & 0 & 1.762895 & -0.639406 & 0.000003 \\
\hline 2 & 1 & 0 & 2.328543 & -0.316591 & 0.880826 \\
\hline 3 & 1 & 0 & 1.695063 & -1.727059 & 0.000044 \\
\hline 4 & 1 & 0 & 2.328560 & -0.316648 & -0.880826 \\
\hline 5 & 6 & 0 & 0.390754 & -0.028429 & -0.000029 \\
\hline 6 & 6 & 0 & 0.239525 & 1.469067 & -0.000002 \\
\hline 7 & 1 & 0 & -0.329647 & 1.792712 & -0.875709 \\
\hline 8 & 1 & 0 & -0.329348 & 1.792730 & 0.875898 \\
\hline 9 & 1 & 0 & 1.210252 & 1.966023 & -0.000144 \\
\hline 10 & 7 & 0 & -0.594929 & -0.840811 & 0.000007 \\
\hline 11 & 8 & 0 & -1.829625 & -0.154166 & 0.000007 \\
\hline 12 & 1 & 0 & -2.460960 & -0.879551 & -0.000032 \\
\hline \multicolumn{6}{|c|}{ Radical - CBS-QB3 } \\
\hline 1 & 6 & 0 & 0.809302 & 1.527896 & 0.508890 \\
\hline 2 & 1 & 0 & 0.588083 & 1.816474 & 1.542292 \\
\hline 3 & 1 & 0 & 1.877990 & 1.330672 & 0.424157 \\
\hline 4 & 1 & 0 & 0.561010 & 2.386187 & -0.125001 \\
\hline 5 & 6 & 0 & 0.010224 & 0.321444 & 0.109604 \\
\hline 6 & 6 & 0 & -1.495594 & 0.364633 & 0.148426 \\
\hline 7 & 1 & 0 & -1.873314 & 1.156291 & -0.507301 \\
\hline 8 & 1 & 0 & -1.920728 & -0.587100 & -0.169167 \\
\hline 9 & 1 & 0 & -1.846659 & 0.587508 & 1.161557 \\
\hline 10 & 7 & 0 & 0.635008 & -0.735687 & -0.261414 \\
\hline 11 & 8 & 0 & 0.278122 & -1.853007 & -0.637270 \\
\hline \multicolumn{6}{|c|}{ Parent - CBS-APNO } \\
\hline 1 & 6 & 0 & 1.767822 & -0.635512 & 0.000000 \\
\hline 2 & 1 & 0 & 2.326974 & -0.306845 & 0.885254 \\
\hline 3 & 1 & 0 & 1.701188 & -1.726408 & 0.000001 \\
\hline 4 & 1 & 0 & 2.326975 & -0.306846 & -0.885253 \\
\hline 5 & 6 & 0 & 0.389147 & -0.029339 & 0.000000 \\
\hline 6 & 6 & 0 & 0.230883 & 1.472623 & 0.000000 \\
\hline 7 & 1 & 0 & -0.336966 & 1.791368 & -0.881019 \\
\hline 8 & 1 & 0 & -0.336951 & 1.791368 & 0.881029 \\
\hline 9 & 1 & 0 & 1.207140 & 1.966122 & -0.000008 \\
\hline 10 & 7 & 0 & -0.598705 & -0.847869 & 0.000000 \\
\hline 11 & 8 & 0 & -1.821615 & -0.155435 & 0.000000 \\
\hline 12 & 1 & 0 & -2.451617 & -0.876827 & 0.000000 \\
\hline \multicolumn{6}{|c|}{ Radical - CBS-APNO } \\
\hline 1 & 6 & 0 & 1.609520 & -0.819573 & 0.000001 \\
\hline 2 & 1 & 0 & 2.211928 & -0.580531 & 0.885402 \\
\hline 3 & 1 & 0 & 1.388903 & -1.890164 & 0.000050 \\
\hline 4 & 1 & 0 & 2.211907 & -0.580605 & -0.885434 \\
\hline 5 & 6 & 0 & 0.334868 & -0.018053 & -0.000011 \\
\hline 6 & 6 & 0 & 0.395112 & 1.491415 & 0.000000 \\
\hline 7 & 1 & 0 & 0.935997 & 1.846840 & -0.885678 \\
\hline 8 & 1 & 0 & -0.610636 & 1.919065 & -0.000259 \\
\hline 9 & 1 & 0 & 0.935535 & 1.846862 & 0.885952 \\
\hline 10 & 7 & 0 & -0.782241 & -0.651829 & 0.000004 \\
\hline 11 & 8 & 0 & -1.954368 & -0.240175 & 0.000000 \\
\hline
\end{tabular}


Table S4. Calculated minimum energy geometries in oximes and their iminoxyl radicals from Table 5 .

\begin{tabular}{|c|c|c|c|c|c|}
\hline \multicolumn{6}{|c|}{ Formaldehyde Oxime - MLM } \\
\hline 1 & 6 & 0 & -1.126690 & 0.177987 & -0.000089 \\
\hline 2 & 1 & 0 & -1.110699 & 1.268761 & 0.000127 \\
\hline 3 & 7 & 0 & -0.070429 & -0.533593 & -0.000026 \\
\hline 4 & 8 & 0 & 1.080467 & 0.267909 & 0.000010 \\
\hline 5 & 1 & 0 & 1.792960 & -0.390670 & 0.000131 \\
\hline 6 & 1 & 0 & -2.072854 & -0.354141 & 0.000381 \\
\hline \multicolumn{6}{|c|}{ Formaldehyde Iminoxyl - MLM } \\
\hline 1 & 6 & 0 & 1.124541 & 0.118487 & 0.000000 \\
\hline 2 & 1 & 0 & 1.301374 & 1.192885 & 0.000000 \\
\hline 3 & 7 & 0 & -0.067104 & -0.352656 & 0.000000 \\
\hline 4 & 8 & 0 & -1.190271 & 0.144207 & 0.000000 \\
\hline 5 & 1 & 0 & 1.943280 & -0.588871 & 0.000001 \\
\hline \multicolumn{6}{|c|}{ Formaldehyde Oxime - CBS-QB3 } \\
\hline 1 & 6 & 0 & -1.125141 & 0.177396 & -0.000042 \\
\hline 2 & 1 & 0 & -1.105573 & 1.266493 & 0.000060 \\
\hline 3 & 7 & 0 & -0.071462 & -0.529238 & -0.000012 \\
\hline 4 & 8 & 0 & 1.079672 & 0.264897 & 0.000005 \\
\hline 5 & 1 & 0 & 1.786746 & -0.388157 & 0.000057 \\
\hline 6 & 1 & 0 & -2.067468 & -0.357225 & 0.000177 \\
\hline \multicolumn{6}{|c|}{ Formaldehyde Iminoxyl - CBS-QB3 } \\
\hline 1 & 6 & 0 & 1.122793 & 0.116373 & 0.000000 \\
\hline 2 & 1 & 0 & 1.299994 & 1.188854 & 0.000000 \\
\hline 3 & 7 & 0 & -0.069595 & -0.347221 & 0.000000 \\
\hline 4 & 8 & 0 & -1.185685 & 0.142184 & 0.000000 \\
\hline 5 & 1 & 0 & 1.935890 & -0.594018 & 0.000001 \\
\hline \multicolumn{6}{|c|}{ Acetaldehyde Oxime - MLM } \\
\hline 1 & 6 & 0 & -0.419943 & 0.375642 & -0.000012 \\
\hline 2 & 1 & 0 & -0.204689 & 1.448138 & -0.000011 \\
\hline 3 & 7 & 0 & 0.532072 & -0.474306 & -0.000014 \\
\hline 4 & 8 & 0 & 1.784844 & 0.172509 & 0.000010 \\
\hline 5 & 1 & 0 & 2.401145 & -0.576338 & 0.000012 \\
\hline 6 & 6 & 0 & -1.841188 & -0.093656 & 0.000007 \\
\hline 7 & 1 & 0 & -2.375673 & 0.281114 & 0.882457 \\
\hline 8 & 1 & 0 & -2.375742 & 0.281261 & -0.882337 \\
\hline 9 & 1 & 0 & -1.881513 & -1.186025 & -0.000078 \\
\hline \multicolumn{6}{|c|}{ Acetaldehyde Iminoxyl - MLM } \\
\hline 1 & 6 & 0 & -0.397155 & 0.446541 & 0.000051 \\
\hline 2 & 1 & 0 & -0.277586 & 1.531842 & -0.000105 \\
\hline 3 & 7 & 0 & 0.660754 & -0.279720 & -0.000114 \\
\hline 4 & 8 & 0 & 1.870296 & -0.032143 & 0.000060 \\
\hline 5 & 6 & 0 & -1.761408 & -0.169859 & -0.000017 \\
\hline 6 & 1 & 0 & -2.333501 & 0.141109 & 0.883499 \\
\hline 7 & 1 & 0 & -2.334300 & 0.143006 & -0.882254 \\
\hline 8 & 1 & 0 & -1.690877 & -1.260863 & -0.001029 \\
\hline
\end{tabular}

\begin{tabular}{cccrrr}
\multicolumn{7}{c}{ Acetaldehyde } & Oxime & Z-isomer - MLM \\
1 & 6 & 0 & 0.520821 & 0.708480 & -0.000001 \\
2 & 1 & 0 & 0.946479 & 1.710436 & -0.000005 \\
3 & 7 & 0 & -0.757850 & 0.698495 & 0.000007 \\
4 & 8 & 0 & -1.251920 & -0.623202 & 0.000001 \\
5 & 1 & 0 & -2.210909 & -0.479504 & -0.000024 \\
6 & 6 & 0 & 1.421853 & -0.492265 & -0.000002 \\
7 & 1 & 0 & 2.473664 & -0.195892 & -0.000294 \\
8 & 1 & 0 & 1.227736 & -1.117963 & 0.879199 \\
9 & 1 & 0 & 1.227294 & -1.118216 & -0.878916
\end{tabular}

Acetaldehyde Iminoxyl Z-isomer - MLM
16
$\begin{array}{llll}0 & 0.468184 & 0.667124 & -0.000030\end{array}$ 


$\begin{array}{rrrrrr}2 & 1 & 0 & 0.780260 & 1.706707 & -0.000009 \\ 3 & 7 & 0 & -0.800933 & 0.478518 & 0.000035 \\ 4 & 8 & 0 & -1.515403 & -0.529213 & -0.000018 \\ 5 & 6 & 0 & 1.482630 & -0.442569 & -0.000014 \\ 6 & 1 & 0 & 2.128755 & -0.377445 & 0.884364 \\ 7 & 1 & 0 & 0.985991 & -1.415934 & -0.000705 \\ 8 & 1 & 0 & 2.129861 & -0.376578 & -0.883488\end{array}$

\begin{tabular}{cccrrr}
\multicolumn{7}{c}{ Acetaldehyde } & Oxime - CBS-QB3 \\
1 & 6 & 0 & -0.419580 & 0.374995 & -0.000010 \\
2 & 1 & 0 & -0.202525 & 1.445603 & -0.000010 \\
3 & 7 & 0 & 0.530261 & -0.469012 & -0.000012 \\
4 & 8 & 0 & 1.783162 & 0.169195 & 0.000009 \\
5 & 1 & 0 & 2.394098 & -0.574066 & 0.000011 \\
6 & 6 & 0 & -1.838802 & -0.094617 & 0.000005 \\
7 & 1 & 0 & -2.370890 & 0.279930 & 0.880695 \\
8 & 1 & 0 & -2.370982 & 0.280149 & -0.880533 \\
9 & 1 & 0 & -1.876532 & -1.184356 & -0.000120
\end{tabular}

\begin{tabular}{cccrrr}
\multicolumn{7}{c}{ Acetaldehyde } & Iminoxyl & - CBS-QB3 & & \\
1 & 6 & 0 & -0.396305 & 0.446925 & 0.000031 \\
2 & 1 & 0 & -0.277339 & 1.530660 & -0.000108 \\
3 & 7 & 0 & 0.661759 & -0.273855 & -0.000131 \\
4 & 8 & 0 & 1.864397 & -0.035494 & 0.000074 \\
5 & 6 & 0 & -1.758216 & -0.171508 & -0.000010 \\
6 & 1 & 0 & -2.328740 & 0.137907 & 0.881775 \\
7 & 1 & 0 & -2.329596 & 0.139543 & -0.880571 \\
8 & 1 & 0 & -1.684687 & -1.259672 & -0.000887
\end{tabular}

Acetaldehyde Oxime Z-isomer - CBS-QB3

$\begin{array}{rrrrrr}1 & 6 & 0 & -0.520575 & 0.685891 & -0.000004 \\ 2 & 1 & 0 & -0.944926 & 1.687616 & -0.000008 \\ 3 & 7 & 0 & 0.753194 & 0.686546 & 0.000006 \\ 4 & 8 & 0 & 1.294370 & -0.613303 & 0.000002 \\ 5 & 1 & 0 & 2.239373 & -0.434969 & -0.000027 \\ 6 & 6 & 0 & -1.449436 & -0.488819 & -0.000001 \\ 7 & 1 & 0 & -2.100279 & -0.453172 & -0.879618 \\ 8 & 1 & 0 & -0.901391 & -1.427937 & -0.000184 \\ 9 & 1 & 0 & -2.100030 & -0.453371 & 0.879811\end{array}$

Acetaldehyde Iminoxyl Z-isomer - CBS-QB3

$\begin{array}{rrrrrr}1 & 6 & 0 & 0.466167 & 0.667236 & 0.000002 \\ 2 & 1 & 0 & 0.774403 & 1.705808 & -0.000047 \\ 3 & 7 & 0 & -0.799604 & 0.472958 & 0.000013 \\ 4 & 8 & 0 & -1.511447 & -0.526326 & -0.000010 \\ 5 & 6 & 0 & 1.480279 & -0.441100 & -0.000011 \\ 6 & 1 & 0 & 2.124898 & -0.374929 & 0.882123 \\ 7 & 1 & 0 & 0.985895 & -1.412800 & 0.000062 \\ 8 & 1 & 0 & 2.124931 & -0.374993 & -0.882098\end{array}$

Trifluoroacetaldehyde Oxime - MLM

$\begin{array}{rrrrrr}1 & 6 & 0 & -0.585341 & -0.480581 & -0.000008 \\ 2 & 1 & 0 & -0.755743 & -1.557148 & -0.000004 \\ 3 & 7 & 0 & -1.519371 & 0.384346 & -0.000008 \\ 4 & 8 & 0 & -2.768229 & -0.220710 & 0.000002 \\ 5 & 1 & 0 & -3.374350 & 0.537981 & 0.000026 \\ 6 & 6 & 0 & 0.841295 & -0.011286 & 0.000000 \\ 7 & 9 & 0 & 0.955937 & 1.321894 & -0.000048 \\ 8 & 9 & 0 & 1.487370 & -0.491787 & -1.086442 \\ 9 & 9 & 0 & 1.487338 & -0.491704 & 1.086498\end{array}$

Trifluoroacetaldehyde Iminoxyl - MLM

$\begin{array}{rllrrr}1 & 6 & 0 & -0.588565 & -0.608451 & 0.000021 \\ 2 & 1 & 0 & -0.690354 & -1.691584 & 0.000069 \\ 3 & 7 & 0 & -1.611923 & 0.163811 & -0.000016 \\ 4 & 8 & 0 & -2.818654 & -0.029605 & 0.000008 \\ 5 & 6 & 0 & 0.783649 & -0.008945 & -0.000001 \\ 6 & 9 & 0 & 0.749954 & 1.331504 & -0.000105 \\ 7 & 9 & 0 & 1.477959 & -0.416615 & -1.084850 \\ 8 & 9 & 0 & 1.477925 & -0.416430 & 1.084938\end{array}$




\begin{tabular}{|c|c|c|c|c|c|}
\hline \multicolumn{6}{|c|}{ Trifluo } \\
\hline 1 & 6 & 0 & -0.416083 & 1.021146 & -0.000010 \\
\hline 2 & 1 & 0 & -0.121407 & 2.065766 & -0.000044 \\
\hline 3 & 7 & 0 & -1.670562 & 0.788014 & 0.000014 \\
\hline 4 & 8 & 0 & -1.965473 & -0.567705 & 0.000006 \\
\hline 5 & 1 & 0 & -2.936272 & -0.575252 & -0.000096 \\
\hline 6 & 6 & 0 & 0.696568 & -0.004081 & -0.000002 \\
\hline 7 & 9 & 0 & 0.657008 & -0.794900 & -1.088032 \\
\hline 8 & 9 & 0 & 0.657201 & -0.794654 & 1.088218 \\
\hline 9 & 9 & 0 & 1.884957 & 0.637626 & -0.00017 \\
\hline
\end{tabular}

Trifluoroacetaldehyde Iminoxyl Z-isomer - MLM

$\begin{array}{rrrrrr}1 & 6 & 0 & -0.461920 & -0.957170 & 0.001996 \\ 2 & 1 & 0 & -0.245094 & -2.017604 & 0.021789 \\ 3 & 7 & 0 & -1.692881 & -0.599110 & 0.009015 \\ 4 & 8 & 0 & -2.292006 & 0.466770 & -0.002582 \\ 5 & 6 & 0 & 0.694547 & 0.008366 & -0.001778 \\ 6 & 9 & 0 & 1.573912 & -0.328739 & -0.967996 \\ 7 & 9 & 0 & 1.360116 & -0.033037 & 1.171861 \\ 8 & 9 & 0 & 0.292145 & 1.269559 & -0.211147\end{array}$

Trifluoroacetaldehyde Oxime - CBS-QB3

$\begin{array}{rrrrrr}1 & 6 & 0 & -0.587491 & -0.479909 & 0.000005 \\ 2 & 1 & 0 & -0.756242 & -1.554913 & 0.000007 \\ 3 & 7 & 0 & -1.518650 & 0.379829 & 0.000003 \\ 4 & 8 & 0 & -2.766637 & -0.215662 & -0.000001 \\ 5 & 1 & 0 & -3.367515 & 0.537510 & -0.000011 \\ 6 & 6 & 0 & 0.837766 & -0.010676 & -0.000001 \\ 7 & 9 & 0 & 0.960463 & 1.319533 & -0.000031 \\ 8 & 9 & 0 & 1.485648 & -0.491604 & -1.085475 \\ 9 & 9 & 0 & 1.485638 & -0.491551 & 1.085503\end{array}$

Trifluoroacetaldehyde Iminoxyl - CBS-QB3

$\begin{array}{rrrrrr}1 & 6 & 0 & -0.590911 & -0.609600 & 0.000000 \\ 2 & 1 & 0 & -0.688343 & -1.691009 & 0.000007 \\ 3 & 7 & 0 & -1.614142 & 0.158428 & -0.000001 \\ 4 & 8 & 0 & -2.812271 & -0.024389 & 0.000001 \\ 5 & 6 & 0 & 0.779025 & -0.009214 & 0.000000 \\ 6 & 9 & 0 & 0.752304 & 1.329689 & -0.000042 \\ 7 & 9 & 0 & 1.477017 & -0.415434 & -1.083223 \\ 8 & 9 & 0 & 1.476992 & -0.415365 & 1.083264\end{array}$

Trifluoroacetaldehyde Oxime Z-isomer - CBS-QB3

$\begin{array}{rrrrrr}1 & 6 & 0 & -0.420917 & 1.017020 & -0.000006 \\ 2 & 1 & 0 & -0.126981 & 2.059580 & 0.000022 \\ 3 & 7 & 0 & -1.670556 & 0.782158 & -0.000012 \\ 4 & 8 & 0 & -1.974348 & -0.566103 & 0.000003 \\ 5 & 1 & 0 & -2.937260 & -0.571612 & 0.000017 \\ 6 & 6 & 0 & 0.695093 & -0.003470 & -0.000002 \\ 7 & 9 & 0 & 0.665420 & -0.794666 & -1.086695 \\ 8 & 9 & 0 & 0.665417 & -0.794644 & 1.086710 \\ 9 & 9 & 0 & 1.881147 & 0.643137 & -0.000006\end{array}$

Trifluoroacetaldehyde Iminoxyl Z-isomer - CBS-QB3

$\begin{array}{rrrrrr}1 & 6 & 0 & -0.467615 & -0.950483 & 0.002780 \\ 2 & 1 & 0 & -0.254343 & -2.009641 & 0.019768 \\ 3 & 7 & 0 & -1.695101 & -0.587499 & 0.007671 \\ 4 & 8 & 0 & -2.303479 & 0.461487 & -0.002140 \\ 5 & 6 & 0 & 0.693704 & 0.006520 & -0.001811 \\ 6 & 9 & 0 & 1.569046 & -0.328932 & -0.972265 \\ 7 & 9 & 0 & 1.366933 & -0.044699 & 1.167299 \\ 8 & 9 & 0 & 0.307504 & 1.272966 & -0.201941\end{array}$

\begin{tabular}{cccrrr}
\multicolumn{7}{c}{ Acetone } & Oxime & - MLM & & & \\
1 & 6 & 0 & 1.764628 & -0.640438 & 0.000003 \\
2 & 1 & 0 & 2.333127 & -0.317815 & 0.882320 \\
3 & 1 & 0 & 1.699866 & -1.731146 & -0.000025 \\
4 & 1 & 0 & 2.333150 & -0.317770 & -0.882282 \\
5 & 6 & 0 & 0.390637 & -0.028614 & 0.000001 \\
6 & 6 & 0 & 0.240856 & 1.471324 & -0.000003
\end{tabular}




$\begin{array}{cccccc}7 & 1 & 0 & -0.327577 & 1.799254 & -0.877867 \\ 8 & 1 & 0 & -0.327426 & 1.799274 & 0.877953 \\ 9 & 1 & 0 & 1.214378 & 1.968947 & -0.000087 \\ 10 & 7 & 0 & -0.597481 & -0.845730 & -0.000010 \\ 11 & 8 & 0 & -1.831412 & -0.151510 & 0.000005 \\ 12 & 1 & 0 & -2.468579 & -0.882185 & 0.000010\end{array}$

Acetone Iminoxyl - MLM

$\begin{array}{cccccc}1 & 6 & 0 & 1.560840 & -0.853282 & 0.040096 \\ 2 & 1 & 0 & 1.938761 & -0.880570 & 1.095392 \\ 3 & 1 & 0 & 1.388772 & -1.904917 & -0.297815 \\ 4 & 1 & 0 & 2.351704 & -0.394709 & -0.604804 \\ 5 & 6 & 0 & 0.335305 & -0.027618 & 0.003506 \\ 6 & 6 & 0 & 0.502416 & 1.440507 & -0.016218 \\ 7 & 1 & 0 & 0.646925 & 1.793227 & -1.070068 \\ 8 & 1 & 0 & -0.395666 & 1.958322 & 0.406232 \\ 9 & 1 & 0 & 1.403533 & 1.733898 & 0.577077 \\ 10 & 7 & 0 & -0.913207 & -0.667337 & -0.156113 \\ 11 & 8 & 0 & -1.916618 & -0.123942 & 0.102809\end{array}$

Benzaldehyde Oxime - MLM

$\begin{array}{cccrrr}1 & 6 & 0 & 2.773173 & 0.251513 & -0.000010 \\ 2 & 6 & 0 & 1.879408 & 1.328390 & 0.000000 \\ 3 & 6 & 0 & 0.507158 & 1.103657 & 0.000011 \\ 4 & 6 & 0 & 0.003335 & -0.209721 & 0.000012 \\ 5 & 6 & 0 & 0.907243 & -1.283235 & 0.000002 \\ 6 & 6 & 0 & 2.282974 & -1.054531 & -0.000009 \\ 7 & 1 & 0 & 3.844690 & 0.432272 & -0.000019 \\ 8 & 1 & 0 & 2.257592 & 2.347296 & 0.000000 \\ 9 & 1 & 0 & -0.190818 & 1.934599 & 0.000019 \\ 10 & 1 & 0 & 2.970155 & -1.896319 & -0.000017 \\ 11 & 6 & 0 & -1.435011 & -0.494214 & 0.000022 \\ 12 & 1 & 0 & -1.754467 & -1.539988 & 0.000037 \\ 13 & 7 & 0 & -2.307470 & 0.444730 & -0.000008 \\ 14 & 8 & 0 & -3.606834 & -0.082196 & -0.000016 \\ 15 & 1 & 0 & -4.156229 & 0.717468 & -0.000007 \\ 16 & 1 & 0 & 0.526369 & -2.302027 & 0.000002\end{array}$

Benzaldehyde Iminoxyl - MLM

$\begin{array}{cccrrr}1 & 6 & 0 & -2.689354 & 0.336088 & 0.000288 \\ 2 & 6 & 0 & -1.742212 & 1.365687 & 0.000128 \\ 3 & 6 & 0 & -0.382997 & 1.072215 & -0.000056 \\ 4 & 6 & 0 & 0.055298 & -0.264559 & -0.000068 \\ 5 & 6 & 0 & -0.902434 & -1.291474 & 0.000086 \\ 6 & 6 & 0 & -2.264046 & -0.992673 & 0.000264 \\ 7 & 1 & 0 & -3.750166 & 0.570474 & 0.000423 \\ 8 & 1 & 0 & -2.067048 & 2.402667 & 0.000133 \\ 9 & 1 & 0 & 0.350841 & 1.873070 & -0.000197 \\ 10 & 1 & 0 & -2.991958 & -1.799355 & 0.000384 \\ 11 & 6 & 0 & 1.471404 & -0.623996 & -0.000248 \\ 12 & 1 & 0 & 1.759551 & -1.676487 & -0.000492 \\ 13 & 7 & 0 & 2.410799 & 0.260070 & -0.000118 \\ 14 & 8 & 0 & 3.640511 & 0.191239 & -0.000232 \\ 15 & 1 & 0 & -0.574849 & -2.328503 & 0.000073\end{array}$

Benzaldehyde Oxime Z-isomer - MLM

$\begin{array}{cccrcc}1 & 6 & 0 & -2.384987 & -1.088479 & 0.000000 \\ 2 & 6 & 0 & -1.145530 & -1.731515 & 0.000000 \\ 3 & 6 & 0 & 0.039247 & -0.997375 & 0.000000 \\ 4 & 6 & 0 & 0.000000 & 0.411741 & 0.000000 \\ 5 & 6 & 0 & -1.258776 & 1.044900 & 0.000000 \\ 6 & 6 & 0 & -2.438332 & 0.306924 & 0.000000 \\ 7 & 6 & 0 & 1.173866 & 1.294936 & 0.000000 \\ 8 & 7 & 0 & 2.442687 & 1.087860 & 0.000000 \\ 9 & 1 & 0 & -1.304765 & 2.131560 & 0.000000 \\ 10 & 1 & 0 & -3.302847 & -1.670335 & 0.000000 \\ 11 & 1 & 0 & -1.098308 & -2.817325 & 0.000000 \\ 12 & 1 & 0 & 0.992593 & -1.506001 & 0.000000 \\ 13 & 1 & 0 & -3.396952 & 0.818623 & 0.000000 \\ 14 & 1 & 0 & 0.950857 & 2.362067 & 0.000000\end{array}$




$\begin{array}{llllll}15 & 8 & 0 & 2.797679 & -0.269674 & 0.000000 \\ 16 & 1 & 0 & 3.766255 & -0.223008 & 0.000000\end{array}$

Benzaldehyde Iminoxyl Z-isomer - MLM

$\begin{array}{cccccc}1 & 6 & 0 & -2.255320 & -1.229739 & 0.000000 \\ 2 & 6 & 0 & -0.971041 & -1.779358 & 0.000000 \\ 3 & 6 & 0 & 0.151621 & -0.954318 & 0.000000 \\ 4 & 6 & 0 & 0.000000 & 0.443575 & 0.000000 \\ 5 & 6 & 0 & -1.297314 & 0.988150 & 0.000000 \\ 6 & 6 & 0 & -2.414566 & 0.158039 & 0.000000 \\ 7 & 6 & 0 & 1.135477 & 1.368583 & 0.000000 \\ 8 & 7 & 0 & 2.384466 & 1.043799 & 0.000000 \\ 9 & 1 & 0 & -1.423000 & 2.068300 & 0.000000 \\ 10 & 1 & 0 & -3.126562 & -1.878873 & 0.000000 \\ 11 & 1 & 0 & -0.841413 & -2.858151 & 0.000000 \\ 12 & 1 & 0 & 1.145607 & -1.388954 & 0.000000 \\ 13 & 1 & 0 & -3.409860 & 0.593993 & 0.000000 \\ 14 & 1 & 0 & 0.947661 & 2.437402 & 0.000000 \\ 15 & 8 & 0 & 2.990395 & -0.031238 & 0.000000 \\ \text { Benzaldehyde Oxime }- \text { CBS-QB3 } & & \\ 1 & 6 & 0 & 2.769088 & 0.251640 & 0.000002 \\ 2 & 6 & 0 & 1.876287 & 1.326167 & 0.000001 \\ 3 & 6 & 0 & 0.506910 & 1.101470 & 0.000000 \\ 4 & 6 & 0 & 0.004009 & -0.209701 & -0.000002 \\ 5 & 6 & 0 & 0.906924 & -1.280788 & 0.000000 \\ 6 & 6 & 0 & 2.280374 & -1.051803 & 0.000001 \\ 7 & 1 & 0 & 3.838017 & 0.432441 & 0.000003 \\ 8 & 1 & 0 & 2.253706 & 2.342672 & 0.000002 \\ 9 & 1 & 0 & -0.189945 & 1.930277 & -0.000001 \\ 10 & 1 & 0 & 2.966923 & -1.890841 & 0.000001 \\ 11 & 6 & 0 & -1.433879 & -0.494017 & -0.000005 \\ 12 & 1 & 0 & -1.754588 & -1.537653 & 0.000006 \\ 13 & 7 & 0 & -2.304216 & 0.439365 & -0.000006 \\ 14 & 8 & 0 & -3.602963 & -0.078431 & 0.000006 \\ 15 & 1 & 0 & -4.147631 & 0.715009 & 0.000010 \\ 16 & 1 & 0 & 0.528451 & -2.297826 & -0.000001\end{array}$

Benzaldehyde Iminoxyl - CBS-QB3

$\begin{array}{cccccc}1 & 6 & 0 & -2.684474 & 0.337304 & 0.000286 \\ 2 & 6 & 0 & -1.737506 & 1.363642 & 0.000155 \\ 3 & 6 & 0 & -0.381359 & 1.069265 & -0.000004 \\ 4 & 6 & 0 & 0.055392 & -0.265546 & -0.000031 \\ 5 & 6 & 0 & -0.902418 & -1.289080 & 0.000099 \\ 6 & 6 & 0 & -2.261370 & -0.989071 & 0.000257 \\ 7 & 1 & 0 & -3.742485 & 0.572532 & 0.000409 \\ 8 & 1 & 0 & -2.060998 & 2.398433 & 0.000173 \\ 9 & 1 & 0 & 0.350450 & 1.868817 & -0.000111 \\ 10 & 1 & 0 & -2.989008 & -1.792608 & 0.000357 \\ 11 & 6 & 0 & 1.470488 & -0.625924 & -0.000193 \\ 12 & 1 & 0 & 1.759079 & -1.676444 & -0.000314 \\ 13 & 7 & 0 & 2.409139 & 0.254949 & -0.000202 \\ 14 & 8 & 0 & 3.630606 & 0.195714 & -0.000325 \\ 15 & 1 & 0 & -0.578372 & -2.324624 & 0.000078\end{array}$

Benzaldehyde Oxime Z-isomer - CBS-QB3

$\begin{array}{rrrrrr}1 & 6 & 0 & -2.383047 & -1.081258 & 0.000000 \\ 2 & 6 & 0 & -1.147259 & -1.725261 & 0.000000 \\ 3 & 6 & 0 & 0.036540 & -0.994550 & 0.000000 \\ 4 & 6 & 0 & 0.000000 & 0.412112 & 0.000000 \\ 5 & 6 & 0 & -1.255548 & 1.046473 & 0.000000 \\ 6 & 6 & 0 & -2.433925 & 0.311523 & 0.000000 \\ 7 & 6 & 0 & 1.176112 & 1.290931 & 0.000000 \\ 8 & 7 & 0 & 2.439408 & 1.077708 & 0.000000 \\ 9 & 1 & 0 & -1.300056 & 2.130683 & 0.000000 \\ 10 & 1 & 0 & -3.299776 & -1.660166 & 0.000000 \\ 11 & 1 & 0 & -1.102617 & -2.808668 & 0.000000 \\ 12 & 1 & 0 & 0.987490 & -1.502305 & 0.000000 \\ 13 & 1 & 0 & -3.389867 & 0.822869 & 0.000000 \\ 14 & 1 & 0 & 0.958142 & 2.356957 & 0.000000 \\ 15 & 8 & 0 & 2.794712 & -0.276347 & 0.000000\end{array}$


$\begin{array}{llllll}16 & 1 & 0 & 3.755903 & -0.232357 & 0.000000\end{array}$

Benzaldehyde Iminoxyl Z-isomer - CBS-QB3

$\begin{array}{rrrrrr}1 & 6 & 0 & -2.249800 & -1.229651 & 0.000000 \\ 2 & 6 & 0 & -0.967486 & -1.776378 & 0.000000 \\ 3 & 6 & 0 & 0.152158 & -0.951471 & 0.000000 \\ 4 & 6 & 0 & 0.000000 & 0.444041 & 0.000000 \\ 5 & 6 & 0 & -1.296387 & 0.985370 & 0.000000 \\ 6 & 6 & 0 & -2.410241 & 0.155291 & 0.000000 \\ 7 & 6 & 0 & 1.134142 & 1.369243 & 0.000000 \\ 8 & 7 & 0 & 2.379451 & 1.038917 & 0.000000 \\ 9 & 1 & 0 & -1.424498 & 2.062720 & 0.000000 \\ 10 & 1 & 0 & -3.118136 & -1.878441 & 0.000000 \\ 11 & 1 & 0 & -0.837238 & -2.852574 & 0.000000 \\ 12 & 1 & 0 & 1.143858 & -1.385459 & 0.000000 \\ 13 & 1 & 0 & -3.404100 & 0.588282 & 0.000000 \\ 14 & 1 & 0 & 0.948813 & 2.436280 & 0.000000 \\ 15 & 8 & 0 & 2.982603 & -0.027737 & 0.000000\end{array}$

\begin{tabular}{cccrrr}
\multicolumn{6}{c}{ Acetophenone } \\
1 & 6 & 0 & -2.594525 & -0.051963 & -1.556769 \\
2 & 6 & 0 & -2.550164 & -0.322629 & -0.185178 \\
3 & 6 & 0 & -1.341911 & -0.284044 & 0.501469 \\
4 & 6 & 0 & -0.142659 & 0.026740 & -0.167600 \\
5 & 6 & 0 & -0.200766 & 0.297130 & -1.543968 \\
6 & 6 & 0 & -1.415063 & 0.257045 & -2.231599 \\
7 & 1 & 0 & -3.540463 & -0.081104 & -2.090890 \\
8 & 1 & 0 & -3.464491 & -0.562190 & 0.351572 \\
9 & 1 & 0 & -1.307567 & -0.491538 & 1.565112 \\
10 & 1 & 0 & -1.434355 & 0.468326 & -3.297521 \\
11 & 6 & 0 & 1.153255 & 0.065865 & 0.561405 \\
12 & 6 & 0 & 2.397662 & 0.550099 & -0.140118 \\
13 & 7 & 0 & 1.124487 & -0.333955 & 1.785843 \\
14 & 8 & 0 & 2.378664 & -0.273164 & 2.414169 \\
15 & 1 & 0 & 2.168139 & -0.592671 & 3.305064 \\
16 & 1 & 0 & 0.704934 & 0.536362 & -2.091690 \\
17 & 1 & 0 & 2.692593 & -0.148798 & -0.932601 \\
18 & 1 & 0 & 2.220488 & 1.523057 & -0.611204 \\
19 & 1 & 0 & 3.225027 & 0.642094 & 0.562056
\end{tabular}

\begin{tabular}{cccccc}
\multicolumn{6}{c}{ Acetophenone Oxime } \\
1 & 6 & 0 & -2.280064 & 0.076600 & -1.690831 \\
2 & 6 & 0 & -2.329456 & 0.338868 & -0.320708 \\
3 & 6 & 0 & -1.167061 & 0.319916 & 0.447510 \\
4 & 6 & 0 & 0.080915 & 0.048427 & -0.145216 \\
5 & 6 & 0 & 0.115607 & -0.196425 & -1.530496 \\
6 & 6 & 0 & -1.050806 & -0.193472 & -2.292931 \\
7 & 1 & 0 & -3.190683 & 0.085109 & -2.283969 \\
8 & 1 & 0 & -3.280621 & 0.558897 & 0.156997 \\
9 & 1 & 0 & -1.220029 & 0.519919 & 1.508995 \\
10 & 1 & 0 & -0.996647 & -0.397726 & -3.358982 \\
11 & 6 & 0 & 1.355344 & 0.067624 & 0.623135 \\
12 & 6 & 0 & 2.625278 & 0.487981 & -0.085032 \\
13 & 7 & 0 & 1.544794 & -0.232505 & 1.862504 \\
14 & 8 & 0 & 0.389235 & -0.667504 & 2.538045 \\
15 & 1 & 0 & 0.745508 & -0.870269 & 3.416860 \\
16 & 1 & 0 & 1.061994 & -0.402272 & -2.020009 \\
17 & 1 & 0 & 2.920981 & -0.241279 & -0.849205 \\
18 & 1 & 0 & 2.496301 & 1.454163 & -0.586342 \\
19 & 1 & 0 & 3.437213 & 0.563906 & 0.641175
\end{tabular}

\begin{tabular}{|c|c|c|c|c|c|}
\hline \multicolumn{6}{|c|}{ Acetophenone Iminoxyl - MLM } \\
\hline 1 & 6 & 0 & -2.535918 & -0.001573 & -1.537741 \\
\hline 2 & 6 & 0 & -2.513126 & -0.000191 & -0.139015 \\
\hline 3 & 6 & & -1.303 & 0.0007 & \\
\hline 4 & 6 & & -0.080633 & 0.000397 & -0.154400 \\
\hline 5 & 6 & 0 & -0.115940 & -0.001096 & -1.558393 \\
\hline 6 & 6 & 0 & -1.332909 & -0.002058 & -2.241546 \\
\hline 7 & 1 & 0 & -3.483011 & -0.002254 & -2.070055 \\
\hline 8 & 1 & 0 & -3.444597 & 0.000275 & 0.420759 \\
\hline
\end{tabular}




$\begin{array}{cccrrr}9 & 1 & 0 & -1.291947 & 0.002007 & 1.630547 \\ 10 & 1 & 0 & -1.335955 & -0.003227 & -3.328256 \\ 11 & 6 & 0 & 1.209481 & 0.001272 & 0.560643 \\ 12 & 6 & 0 & 2.532882 & 0.005360 & -0.169461 \\ 13 & 7 & 0 & 1.193915 & -0.002013 & 1.854403 \\ 14 & 8 & 0 & 2.076931 & -0.002175 & 2.717118 \\ 15 & 1 & 0 & 0.808378 & -0.001728 & -2.127204 \\ 16 & 1 & 0 & 2.630908 & -0.880409 & -0.808249 \\ 17 & 1 & 0 & 2.623404 & 0.889588 & -0.811359 \\ 18 & 1 & 0 & 3.355922 & 0.009967 & 0.547565\end{array}$

Acetophenone Iminoxyl (Z-isomer) - MLM

$\begin{array}{rrrrrr}1 & 6 & 0 & -2.329392 & -0.000455 & -1.603990 \\ 2 & 6 & 0 & -2.348466 & -0.001533 & -0.207992 \\ 3 & 6 & 0 & -1.158795 & -0.001227 & 0.516335 \\ 4 & 6 & 0 & 0.083778 & 0.000175 & -0.145353 \\ 5 & 6 & 0 & 0.088847 & 0.001240 & -1.553869 \\ 6 & 6 & 0 & -1.104499 & 0.000933 & -2.272439 \\ 7 & 1 & 0 & -3.259760 & -0.000696 & -2.165150 \\ 8 & 1 & 0 & -3.295910 & -0.002622 & 0.324022 \\ 9 & 1 & 0 & -1.191901 & -0.002080 & 1.599787 \\ 10 & 1 & 0 & -1.074734 & 0.001782 & -3.358764 \\ 11 & 6 & 0 & 1.367998 & 0.000535 & 0.587044 \\ 12 & 6 & 0 & 2.691551 & 0.001814 & -0.136357 \\ 13 & 7 & 0 & 1.436571 & -0.000312 & 1.879898 \\ 14 & 8 & 0 & 0.610930 & -0.001482 & 2.799878 \\ 15 & 1 & 0 & 1.029188 & 0.002324 & -2.094614 \\ 16 & 1 & 0 & 2.797729 & -0.882210 & -0.776548 \\ 17 & 1 & 0 & 2.796764 & 0.886945 & -0.775178 \\ 18 & 1 & 0 & 3.509048 & 0.001704 & 0.587871\end{array}$

\begin{tabular}{cccccc}
\multicolumn{7}{c}{ Acetophenone } & Oxime & - & CBS-QB3 & & \\
1 & 6 & 0 & -3.014735 & -0.210235 & -0.014538 \\
2 & 6 & 0 & -2.196970 & -1.339286 & 0.062988 \\
3 & 6 & 0 & -0.815814 & -1.207478 & 0.075207 \\
4 & 6 & 0 & -0.213854 & 0.060954 & 0.009647 \\
5 & 6 & 0 & -1.045694 & 1.186130 & -0.066302 \\
6 & 6 & 0 & -2.432888 & 1.051033 & -0.079627 \\
7 & 1 & 0 & -4.093697 & -0.316006 & -0.022249 \\
8 & 1 & 0 & -2.641000 & -2.327156 & 0.117430 \\
9 & 1 & 0 & -0.181499 & -2.081780 & 0.137001 \\
10 & 1 & 0 & -3.056312 & 1.936046 & -0.141293 \\
11 & 6 & 0 & 1.266224 & 0.208203 & 0.016739 \\
12 & 6 & 0 & 1.886686 & 1.576342 & 0.129052 \\
13 & 7 & 0 & 1.941898 & -0.879050 & -0.079317 \\
14 & 8 & 0 & 3.327755 & -0.674896 & -0.066991 \\
15 & 1 & 0 & 3.662742 & -1.573899 & -0.138040 \\
16 & 1 & 0 & -0.616705 & 2.178693 & -0.121267 \\
17 & 1 & 0 & 1.691021 & 2.162079 & -0.774521 \\
18 & 1 & 0 & 1.460366 & 2.122431 & 0.974031 \\
19 & 1 & 0 & 2.962032 & 1.498125 & 0.261061
\end{tabular}

Acetophenone Iminoxyl - CBS-QB3

$\begin{array}{cccccc}1 & 6 & 0 & -2.952573 & -0.228378 & -0.000283 \\ 2 & 6 & 0 & -2.119636 & -1.349279 & 0.001416 \\ 3 & 6 & 0 & -0.740894 & -1.199764 & 0.001688 \\ 4 & 6 & 0 & -0.155342 & 0.078742 & 0.000165 \\ 5 & 6 & 0 & -1.001983 & 1.196334 & -0.001484 \\ 6 & 6 & 0 & -2.386817 & 1.041975 & -0.001723 \\ 7 & 1 & 0 & -4.029838 & -0.347981 & -0.000426 \\ 8 & 1 & 0 & -2.549726 & -2.344571 & 0.002640 \\ 9 & 1 & 0 & -0.100972 & -2.074292 & 0.003153 \\ 10 & 1 & 0 & -3.022112 & 1.920553 & -0.003066 \\ 11 & 6 & 0 & 1.309169 & 0.249899 & 0.000341 \\ 12 & 6 & 0 & 1.959827 & 1.612228 & 0.002759 \\ 13 & 7 & 0 & 2.050080 & -0.807705 & -0.001644 \\ 14 & 8 & 0 & 3.261881 & -0.998033 & -0.001918 \\ 15 & 1 & 0 & -0.585414 & 2.195843 & -0.002744 \\ 16 & 1 & 0 & 1.666959 & 2.185383 & -0.881692 \\ 17 & 1 & 0 & 1.661183 & 2.184844 & 0.885570\end{array}$


Acetophenone Oxime Z-isomer - CBS-QB3

$\begin{array}{rrrrrr}1 & 6 & 0 & 2.821638 & -0.215322 & -0.016204 \\ 2 & 6 & 0 & 1.978203 & -1.236343 & 0.416833 \\ 3 & 6 & 0 & 0.599834 & -1.052902 & 0.439493 \\ 4 & 6 & 0 & 0.034068 & 0.169445 & 0.041247 \\ 5 & 6 & 0 & 0.897728 & 1.194074 & -0.376889 \\ 6 & 6 & 0 & 2.274976 & 1.001165 & -0.416212 \\ 7 & 1 & 0 & 3.895061 & -0.366160 & -0.040056 \\ 8 & 1 & 0 & 2.394907 & -2.184887 & 0.736910 \\ 9 & 1 & 0 & -0.044962 & -1.854714 & 0.765544 \\ 10 & 1 & 0 & 2.920226 & 1.803850 & -0.755252 \\ 11 & 6 & 0 & -1.431437 & 0.423402 & 0.095947 \\ 12 & 6 & 0 & -1.914168 & 1.809483 & 0.455625 \\ 13 & 7 & 0 & -2.382018 & -0.410523 & -0.125638 \\ 14 & 8 & 0 & -1.955254 & -1.696847 & -0.495241 \\ 15 & 1 & 0 & -2.793681 & -2.142007 & -0.651267 \\ 16 & 1 & 0 & 0.492848 & 2.149696 & -0.686181 \\ 17 & 1 & 0 & -1.463847 & 2.148798 & 1.392645 \\ 18 & 1 & 0 & -1.650942 & 2.537142 & -0.317897 \\ 19 & 1 & 0 & -2.998502 & 1.798699 & 0.557921\end{array}$

Acetophenone Iminoxyl Z-isomer - CBS-QB3

$\begin{array}{rrrrrr}1 & 6 & 0 & 2.813625 & -0.238679 & 0.000005 \\ 2 & 6 & 0 & 1.944145 & -1.327333 & 0.000020 \\ 3 & 6 & 0 & 0.567555 & -1.132686 & 0.000013 \\ 4 & 6 & 0 & 0.026842 & 0.164921 & -0.000010 \\ 5 & 6 & 0 & 0.916473 & 1.254453 & -0.000021 \\ 6 & 6 & 0 & 2.292094 & 1.052698 & -0.000016 \\ 7 & 1 & 0 & 3.886171 & -0.395097 & 0.000010 \\ 8 & 1 & 0 & 2.338225 & -2.337171 & 0.000039 \\ 9 & 1 & 0 & -0.091058 & -1.990574 & 0.000026 \\ 10 & 1 & 0 & 2.957588 & 1.908565 & -0.000027 \\ 11 & 6 & 0 & -1.429306 & 0.414145 & -0.000016 \\ 12 & 6 & 0 & -1.992837 & 1.812125 & 0.000032 \\ 13 & 7 & 0 & -2.297599 & -0.543094 & -0.000010 \\ 14 & 8 & 0 & -2.244312 & -1.769942 & -0.000015 \\ 15 & 1 & 0 & 0.535576 & 2.267418 & -0.000035 \\ 16 & 1 & 0 & -1.669552 & 2.370658 & 0.883298 \\ 17 & 1 & 0 & -1.669603 & 2.370699 & -0.883227 \\ 18 & 1 & 0 & -3.081216 & 1.768829 & 0.000061\end{array}$

Benzophenone Oxime - MLM

\begin{tabular}{|c|c|c|c|c|c|}
\hline 1 & 6 & 0 & -3.630214 & -0.867615 & . 463920 \\
\hline 2 & 6 & 0 & -3.600006 & -1.140793 & -0.093384 \\
\hline 3 & 6 & 0 & -2.411130 & -1.022780 & 0.620722 \\
\hline 4 & 6 & 0 & -1.224293 & -0.631078 & -0.023759 \\
\hline 5 & 6 & 0 & -1.266235 & -0.356625 & -1.400276 \\
\hline 6 & 6 & 0 & -2.459291 & -0.476293 & -2.113400 \\
\hline 7 & 1 & 0 & -4.560624 & -0.955424 & -2.018625 \\
\hline 8 & 1 & 0 & -4.509159 & -1.439148 & 0.422188 \\
\hline 9 & 1 & 0 & -2.384653 & -1.228873 & 1.685240 \\
\hline 10 & 1 & 0 & -2.470538 & -0.265134 & -3.179432 \\
\hline 11 & 6 & 0 & 0.055002 & -0.538133 & 0.734207 \\
\hline 12 & 6 & 0 & 1.126875 & 0.390691 & 0.268252 \\
\hline 13 & 6 & 0 & 2.449584 & -0.046370 & 0.097335 \\
\hline 14 & 6 & 0 & 3.431905 & 0.834246 & 2297 \\
\hline 15 & 6 & 0 & 3.112862 & 2.166447 & -0.624276 \\
\hline 16 & 6 & 0 & 1.801567 & 2.612503 & -0.452039 \\
\hline 17 & 6 & 0 & 0.814052 & 1.728897 & -0.017561 \\
\hline 18 & 1 & 0 & 2.703655 & -1.076483 & 0.320955 \\
\hline 19 & 1 & 0 & 4.450337 & 0.479679 & -0.487027 \\
\hline 20 & 1 & 0 & 3.881953 & 2.852428 & -0.969355 \\
\hline 21 & 1 & 0 & 1.544684 & 3.647839 & -0.659721 \\
\hline 22 & 7 & 0 & 0.129577 & -1.296256 & 1.777020 \\
\hline 23 & 8 & 0 & 1.324954 & -1.138899 & 2.494387 \\
\hline 24 & 1 & 0 & 1.200019 & -1.767555 & 3.222621 \\
\hline 25 & 1 & 0 & -0.358993 & -0.057728 & -1.915442 \\
\hline 26 & 1 & 0 & -0.207421 & 2.076795 & 0.106741 \\
\hline
\end{tabular}




\begin{tabular}{cccrrr}
\multicolumn{6}{c}{ Benzophenone } \\
1 & 6 & 0 & -3.899920 & -0.985114 & 0.125689 \\
2 & 6 & 0 & -3.698003 & 0.198558 & -0.587654 \\
3 & 6 & 0 & -2.433914 & 0.781779 & -0.636143 \\
4 & 6 & 0 & -1.348149 & 0.195598 & 0.035630 \\
5 & 6 & 0 & -1.562308 & -0.992966 & 0.752705 \\
6 & 6 & 0 & -2.827667 & -1.577321 & 0.794978 \\
7 & 1 & 0 & -4.884844 & -1.442813 & 0.158038 \\
8 & 1 & 0 & -4.525197 & 0.664776 & -1.116144 \\
9 & 1 & 0 & -2.275665 & 1.695934 & -1.200583 \\
10 & 1 & 0 & -2.976064 & -2.493778 & 1.359779 \\
11 & 6 & 0 & -0.015207 & 0.851653 & 0.022497 \\
12 & 6 & 0 & 1.261621 & 0.092898 & -0.026652 \\
13 & 6 & 0 & 2.449264 & 0.630876 & 0.498440 \\
14 & 6 & 0 & 3.641341 & -0.087299 & 0.432742 \\
15 & 6 & 0 & 3.674848 & -1.347650 & -0.167277 \\
16 & 6 & 0 & 2.503651 & -1.886279 & -0.702491 \\
17 & 6 & 0 & 1.306853 & -1.175839 & -0.632327 \\
18 & 1 & 0 & 2.437822 & 1.612325 & 0.960140 \\
19 & 1 & 0 & 4.547727 & 0.341950 & 0.850916 \\
20 & 1 & 0 & 4.607277 & -1.902834 & -0.221768 \\
21 & 1 & 0 & 2.520047 & -2.861248 & -1.182144 \\
22 & 7 & 0 & -0.027324 & 2.150319 & 0.051954 \\
23 & 8 & 0 & 0.844975 & 3.023886 & 0.042102 \\
24 & 1 & 0 & -0.738247 & -1.451667 & 1.290352 \\
25 & 1 & 0 & 0.404151 & -1.599330 & -1.059908
\end{tabular}

Fluorenone Oxime - MLM

$\begin{array}{rrrrrr}1 & 6 & 0 & -3.477292 & -0.001011 & -0.651696 \\ 2 & 6 & 0 & -3.483362 & -0.001762 & 0.748038 \\ 3 & 6 & 0 & -2.283162 & -0.001675 & 1.466195 \\ 4 & 6 & 0 & -1.086754 & -0.000829 & 0.757254 \\ 5 & 6 & 0 & -1.074949 & -0.000069 & -0.653991 \\ 6 & 6 & 0 & -2.273349 & -0.000160 & -1.364164 \\ 7 & 1 & 0 & -4.419954 & -0.001093 & -1.192454 \\ 8 & 1 & 0 & -4.429905 & -0.002416 & 1.281579 \\ 9 & 1 & 0 & -2.278063 & -0.002255 & 2.551962 \\ 10 & 1 & 0 & -2.279693 & 0.000422 & -2.451063 \\ 11 & 6 & 0 & 0.314140 & -0.000538 & 1.235747 \\ 12 & 6 & 0 & 1.175738 & 0.000448 & 0.032311 \\ 13 & 6 & 0 & 2.562149 & 0.001070 & -0.118853 \\ 14 & 6 & 0 & 3.096372 & 0.001964 & -1.412730 \\ 15 & 6 & 0 & 2.260218 & 0.002234 & -2.532701 \\ 16 & 6 & 0 & 0.868519 & 0.001614 & -2.386654 \\ 17 & 6 & 0 & 0.327590 & 0.000721 & -1.104683 \\ 18 & 1 & 0 & 3.210067 & 0.000862 & 0.748185 \\ 19 & 1 & 0 & 4.174690 & 0.002455 & -1.545977 \\ 20 & 1 & 0 & 2.694839 & 0.002932 & -3.528865 \\ 21 & 1 & 0 & 0.225504 & 0.001832 & -3.262923 \\ 22 & 7 & 0 & 0.573808 & -0.001113 & 2.497510 \\ 23 & 8 & 0 & 1.945348 & -0.000718 & 2.762519 \\ 24 & 1 & 0 & 1.967913 & -0.001233 & 3.732388\end{array}$

Fluorenone Iminoxyl - MLM

$\begin{array}{cccccc}1 & 6 & 0 & -3.454943 & 0.000038 & -0.634684 \\ 2 & 6 & 0 & -3.462026 & 0.000062 & 0.765550 \\ 3 & 6 & 0 & -2.263838 & 0.000058 & 1.485369 \\ 4 & 6 & 0 & -1.065277 & 0.000029 & 0.777773 \\ 5 & 6 & 0 & -1.050964 & 0.000004 & -0.638034 \\ 6 & 6 & 0 & -2.251210 & 0.000009 & -1.345997 \\ 7 & 1 & 0 & -4.397215 & 0.000043 & -1.175694 \\ 8 & 1 & 0 & -4.408474 & 0.000083 & 1.298855 \\ 9 & 1 & 0 & -2.267076 & 0.000077 & 2.571740 \\ 10 & 1 & 0 & -2.257094 & -0.000011 & -2.432787 \\ 11 & 6 & 0 & 0.330885 & 0.000016 & 1.235177 \\ 12 & 6 & 0 & 1.194674 & -0.000016 & 0.043741 \\ 13 & 6 & 0 & 2.580115 & -0.000036 & -0.079957 \\ 14 & 6 & 0 & 3.129570 & -0.000066 & -1.366487 \\ 15 & 6 & 0 & 2.302249 & -0.000075 & -2.495425 \\ 16 & 6 & 0 & 0.908860 & -0.000054 & -2.368380\end{array}$




$\begin{array}{rrrrrr}17 & 6 & 0 & 0.349089 & -0.000024 & -1.092915 \\ 18 & 1 & 0 & 3.216607 & -0.000030 & 0.799990 \\ 19 & 1 & 0 & 4.208817 & -0.000083 & -1.489508 \\ 20 & 1 & 0 & 2.748595 & -0.000099 & -3.486134 \\ 21 & 1 & 0 & 0.278538 & -0.000060 & -3.253745 \\ 22 & 7 & 0 & 0.703123 & 0.000031 & 2.474646 \\ 23 & 8 & 0 & 1.809041 & 0.000025 & 3.016296\end{array}$

Diethyl ketone Oxime - MLM

$\begin{array}{cccrrr}1 & 6 & 0 & -1.393077 & -0.851994 & -0.191194 \\ 2 & 1 & 0 & -1.211691 & -1.726084 & 0.453409 \\ 3 & 1 & 0 & -1.569483 & -1.271597 & -1.193576 \\ 4 & 6 & 0 & -0.113155 & -0.048795 & -0.247458 \\ 5 & 6 & 0 & 1.163734 & -0.762913 & -0.631664 \\ 6 & 1 & 0 & 0.910450 & -1.660765 & -1.208546 \\ 7 & 1 & 0 & 1.755199 & -0.107845 & -1.279616 \\ 8 & 7 & 0 & -0.188002 & 1.193850 & 0.057364 \\ 9 & 8 & 0 & 1.074929 & 1.832286 & -0.023812 \\ 10 & 6 & 0 & 2.006558 & -1.156410 & 0.596379 \\ 11 & 1 & 0 & 2.299592 & -0.265589 & 1.158615 \\ 12 & 1 & 0 & 1.448584 & -1.818732 & 1.268437 \\ 13 & 1 & 0 & 2.915848 & -1.682967 & 0.285630 \\ 14 & 6 & 0 & -2.635095 & -0.098152 & 0.286386 \\ 15 & 1 & 0 & -2.492875 & 0.296161 & 1.297089 \\ 16 & 1 & 0 & -2.856013 & 0.751230 & -0.366656 \\ 17 & 1 & 0 & -3.503881 & -0.765454 & 0.293363 \\ 18 & 1 & 0 & 0.847065 & 2.745989 & 0.206117\end{array}$

Diethyl ketone Iminoxyl - MLM

$\begin{array}{cccccc}\text { Diethyl ketone } & \text { Iminoxyl } & - \text { MLM } \\ 1 & 6 & 0 & -1.336150 & -0.934694 & -0.139594 \\ 2 & 1 & 0 & -1.139537 & -1.758440 & 0.563798 \\ 3 & 1 & 0 & -1.509244 & -1.423476 & -1.110524 \\ 4 & 6 & 0 & -0.084728 & -0.101262 & -0.249366 \\ 5 & 6 & 0 & 1.243568 & -0.736066 & -0.614289 \\ 6 & 1 & 0 & 1.052640 & -1.720227 & -1.058186 \\ 7 & 1 & 0 & 1.724905 & -0.119224 & -1.384203 \\ 8 & 7 & 0 & -0.141433 & 1.159841 & -0.003244 \\ 9 & 8 & 0 & 0.721205 & 2.054005 & -0.017634 \\ 10 & 6 & 0 & 2.188917 & -0.871999 & 0.591596 \\ 11 & 1 & 0 & 2.395916 & 0.109417 & 1.030179 \\ 12 & 1 & 0 & 1.753112 & -1.508817 & 1.369489 \\ 13 & 1 & 0 & 3.142760 & -1.315645 & 0.285317 \\ 14 & 6 & 0 & -2.589841 & -0.170401 & 0.289999 \\ 15 & 1 & 0 & -2.454254 & 0.290336 & 1.273896 \\ 16 & 1 & 0 & -2.827428 & 0.628509 & -0.419934 \\ 17 & 1 & 0 & -3.449076 & -0.846836 & 0.343871\end{array}$

Diethyl ketone Oxime - CBS-QB3

$\begin{array}{cccrrr}\text { Diethyl ketone } & \text { Oxime } & - \text { CBS-QB3 } & & \\ 1 & 6 & 0 & -1.397557 & -0.845868 & -0.191700 \\ 2 & 1 & 0 & -1.217754 & -1.721143 & 0.447211 \\ 3 & 1 & 0 & -1.573075 & -1.257989 & -1.194606 \\ 4 & 6 & 0 & -0.114989 & -0.049822 & -0.245683 \\ 5 & 6 & 0 & 1.157218 & -0.768000 & -0.629849 \\ 6 & 1 & 0 & 0.898850 & -1.666517 & -1.198188 \\ 7 & 1 & 0 & 1.742858 & -0.117102 & -1.283001 \\ 8 & 7 & 0 & -0.180554 & 1.189318 & 0.054798 \\ 9 & 8 & 0 & 1.081514 & 1.823471 & -0.025385 \\ 10 & 6 & 0 & 2.006046 & -1.151742 & 0.596204 \\ 11 & 1 & 0 & 2.302275 & -0.257564 & 1.146092 \\ 12 & 1 & 0 & 1.452486 & -1.806093 & 1.275445 \\ 13 & 1 & 0 & 2.911030 & -1.680458 & 0.285756 \\ 14 & 6 & 0 & -2.636261 & -0.091287 & 0.288771 \\ 15 & 1 & 0 & -2.493941 & 0.295337 & 1.299742 \\ 16 & 1 & 0 & -2.852635 & 0.761165 & -0.357446 \\ 17 & 1 & 0 & -3.505607 & -0.753890 & 0.290743 \\ 18 & 1 & 0 & 0.860538 & 2.731578 & 0.201291\end{array}$

Diethyl ketone Iminoxyl - CBS-QB3

$\begin{array}{llllll}1 & 6 & 0 & -1.336794 & -0.931155 & -0.140738 \\ 2 & 1 & 0 & -1.139583 & -1.755763 & 0.557439\end{array}$




$\begin{array}{cccccc}3 & 1 & 0 & -1.508333 & -1.413066 & -1.112596 \\ 4 & 6 & 0 & -0.084984 & -0.100415 & -0.247223 \\ 5 & 6 & 0 & 1.240019 & -0.738402 & -0.611970 \\ 6 & 1 & 0 & 1.045313 & -1.721859 & -1.049147 \\ 7 & 1 & 0 & 1.716704 & -0.126802 & -1.385575 \\ 8 & 7 & 0 & -0.135774 & 1.158669 & -0.005104 \\ 9 & 8 & 0 & 0.720385 & 2.048799 & -0.018553 \\ 10 & 6 & 0 & 2.189151 & -0.869148 & 0.590373 \\ 11 & 1 & 0 & 2.400992 & 0.111899 & 1.020962 \\ 12 & 1 & 0 & 1.755311 & -1.498674 & 1.371738 \\ 13 & 1 & 0 & 3.138615 & -1.316174 & 0.284481 \\ 14 & 6 & 0 & -2.589499 & -0.169603 & 0.291604 \\ 15 & 1 & 0 & -2.455279 & 0.284974 & 1.275783 \\ 16 & 1 & 0 & -2.826503 & 0.630964 & -0.412758 \\ 17 & 1 & 0 & -3.447258 & -0.844233 & 0.341553\end{array}$

Di-iso-propyl ketone Oxime - MLM

$\begin{array}{cccccc}1 & 6 & 0 & 0.000000 & 0.000000 & 0.000000 \\ 2 & 1 & 0 & 0.000000 & 0.000000 & 1.097098 \\ 3 & 6 & 0 & 1.463024 & 0.000000 & -0.453105 \\ 4 & 6 & 0 & 2.579921 & 0.092586 & 0.574986 \\ 5 & 1 & 0 & 3.521941 & 0.124281 & 0.022899 \\ 6 & 7 & 0 & 1.633253 & -0.084653 & -1.720706 \\ 7 & 8 & 0 & 2.995266 & -0.079216 & -2.106878 \\ 8 & 1 & 0 & 2.921398 & -0.146630 & -3.070976 \\ 9 & 6 & 0 & 2.477633 & 1.384015 & 1.409172 \\ 10 & 1 & 0 & 1.556062 & 1.418857 & 2.002179 \\ 11 & 1 & 0 & 3.320302 & 1.446223 & 2.107794 \\ 12 & 1 & 0 & 2.504139 & 2.273652 & 0.770461 \\ 13 & 6 & 0 & 2.604054 & -1.156891 & 1.477695 \\ 14 & 1 & 0 & 1.683824 & -1.254356 & 2.065896 \\ 15 & 1 & 0 & 2.727588 & -2.070586 & 0.886375 \\ 16 & 1 & 0 & 3.440962 & -1.095325 & 2.183344 \\ 17 & 6 & 0 & -0.729102 & 1.273217 & -0.469337 \\ 18 & 1 & 0 & -0.750489 & 1.316259 & -1.563056 \\ 19 & 1 & 0 & -1.761966 & 1.284902 & -0.101086 \\ 20 & 1 & 0 & -0.230399 & 2.179260 & -0.107035 \\ 21 & 6 & 0 & -0.738858 & -1.265687 & -0.469967 \\ 22 & 1 & 0 & -1.773795 & -1.265511 & -0.107341 \\ 23 & 1 & 0 & -0.753430 & -1.314266 & -1.563044 \\ 24 & 1 & 0 & -0.249716 & -2.173967 & -0.100225\end{array}$

Di-iso-propyl iminoxyl - MLM

$\begin{array}{rrrrrr}1 & 6 & 0 & 1.139538 & -0.684516 & 0.169368 \\ 2 & 1 & 0 & 0.679662 & -1.624354 & 0.499762 \\ 3 & 6 & 0 & 0.002380 & 0.308505 & -0.036477 \\ 4 & 6 & 0 & -1.464859 & -0.114109 & -0.013119 \\ 5 & 1 & 0 & -2.042890 & 0.780859 & -0.271122 \\ 6 & 7 & 0 & 0.312694 & 1.540435 & -0.234315 \\ 7 & 8 & 0 & -0.336311 & 2.576942 & -0.438547 \\ 8 & 6 & 0 & -1.768259 & -1.198405 & -1.062599 \\ 9 & 1 & 0 & -1.213311 & -2.122864 & -0.863412 \\ 10 & 1 & 0 & -2.835993 & -1.446731 & -1.048016 \\ 11 & 1 & 0 & -1.514225 & -0.859387 & -2.072573 \\ 12 & 6 & 0 & -1.889967 & -0.565457 & 1.397298 \\ 13 & 1 & 0 & -1.339570 & -1.457764 & 1.718627 \\ 14 & 1 & 0 & -1.718278 & 0.224685 & 2.135783 \\ 15 & 1 & 0 & -2.957550 & -0.814982 & 1.408486 \\ 16 & 6 & 0 & 1.888265 & -0.965582 & -1.148701 \\ 17 & 1 & 0 & 2.365310 & -0.051110 & -1.518930 \\ 18 & 1 & 0 & 2.668628 & -1.720661 & -0.996805 \\ 19 & 1 & 0 & 1.210611 & -1.330005 & -1.928207 \\ 20 & 6 & 0 & 2.112282 & -0.223711 & 1.268972 \\ 21 & 1 & 0 & 2.901147 & -0.968873 & 1.423781 \\ 22 & 1 & 0 & 2.587664 & 0.723288 & 0.990841 \\ 23 & 1 & 0 & 1.594142 & -0.071027 & 2.221913\end{array}$

Di-iso-propyl ketone Oxime - CBS-QB3

$\begin{array}{llllll}1 & 6 & 0 & 1.223934 & -0.595731 & 0.092600 \\ 2 & 1 & 0 & 0.860773 & -1.612394 & 0.273810\end{array}$




$\begin{array}{cccccc}3 & 6 & 0 & -0.006616 & 0.306278 & -0.026930 \\ 4 & 6 & 0 & -1.400084 & -0.299166 & -0.012119 \\ 5 & 1 & 0 & -2.100370 & 0.518759 & -0.179952 \\ 6 & 7 & 0 & 0.247383 & 1.553388 & -0.127166 \\ 7 & 8 & 0 & -0.905867 & 2.361970 & -0.237963 \\ 8 & 1 & 0 & -0.520711 & 3.240639 & -0.303563 \\ 9 & 6 & 0 & -1.591040 & -1.325940 & -1.142959 \\ 10 & 1 & 0 & -0.927917 & -2.188519 & -1.031325 \\ 11 & 1 & 0 & -2.619086 & -1.699151 & -1.136434 \\ 12 & 1 & 0 & -1.404182 & -0.877698 & -2.121973 \\ 13 & 6 & 0 & -1.714318 & -0.913789 & 1.365193 \\ 14 & 1 & 0 & -1.037592 & -1.738514 & 1.607853 \\ 15 & 1 & 0 & -1.635612 & -0.165319 & 2.157334 \\ 16 & 1 & 0 & -2.733886 & -1.309764 & 1.375618 \\ 17 & 6 & 0 & 2.031037 & -0.612060 & -1.218223 \\ 18 & 1 & 0 & 2.411178 & 0.387965 & -1.438651 \\ 19 & 1 & 0 & 2.880659 & -1.296702 & -1.138085 \\ 20 & 1 & 0 & 1.417758 & -0.932619 & -2.064353 \\ 21 & 6 & 0 & 2.110585 & -0.195390 & 1.283528 \\ 22 & 1 & 0 & 2.964765 & -0.873493 & 1.370652 \\ 23 & 1 & 0 & 2.485222 & 0.821529 & 1.152947 \\ 24 & 1 & 0 & 1.553271 & -0.229408 & 2.223432\end{array}$

Di-iso-propyl ketone Iminoxyl - CBS-QB3

$\begin{array}{rrrrrr}1 & 6 & 0 & 1.140372 & -0.692832 & 0.109999 \\ 2 & 1 & 0 & 0.679657 & -1.663934 & 0.317871 \\ 3 & 6 & 0 & 0.001130 & 0.308106 & -0.027645 \\ 4 & 6 & 0 & -1.463722 & -0.115642 & -0.011612 \\ 5 & 1 & 0 & -2.042248 & 0.795883 & -0.186735 \\ 6 & 7 & 0 & 0.307292 & 1.546685 & -0.158422 \\ 7 & 8 & 0 & -0.343758 & 2.587078 & -0.292678 \\ 8 & 6 & 0 & -1.784281 & -1.108489 & -1.141697 \\ 9 & 1 & 0 & -1.231617 & -2.045439 & -1.026514 \\ 10 & 1 & 0 & -2.850228 & -1.353229 & -1.133949 \\ 11 & 1 & 0 & -1.542325 & -0.688648 & -2.120911 \\ 12 & 6 & 0 & -1.866375 & -0.677877 & 1.363074 \\ 13 & 1 & 0 & -1.313931 & -1.591174 & 1.603513 \\ 14 & 1 & 0 & -1.683219 & 0.049358 & 2.157512 \\ 15 & 1 & 0 & -2.931602 & -0.926190 & 1.370859 \\ 16 & 6 & 0 & 1.938892 & -0.823199 & -1.199943 \\ 17 & 1 & 0 & 2.417491 & 0.127434 & -1.449721 \\ 18 & 1 & 0 & 2.719290 & -1.583534 & -1.102532 \\ 19 & 1 & 0 & 1.294690 & -1.104055 & -2.036713 \\ 20 & 6 & 0 & 2.064352 & -0.349545 & 1.290516 \\ 21 & 1 & 0 & 2.851996 & -1.101204 & 1.395523 \\ 22 & 1 & 0 & 2.539576 & 0.622463 & 1.135199 \\ 23 & 1 & 0 & 1.509282 & -0.304277 & 2.230828\end{array}$

Iso-propyl Tert-butyl Ketone Oxime - MLM

$\begin{array}{rrrrrr}1 & 6 & 0 & -0.379539 & -0.097336 & -1.236772 \\ 2 & 6 & 0 & -0.334788 & 0.002476 & 0.303771 \\ 3 & 6 & 0 & 0.909626 & -0.201987 & 1.175319 \\ 4 & 7 & 0 & -1.458714 & 0.333594 & 0.833998 \\ 5 & 8 & 0 & -1.401172 & 0.451971 & 2.243854 \\ 6 & 1 & 0 & -2.322055 & 0.670634 & 2.453769 \\ 7 & 6 & 0 & 1.933677 & -1.259249 & 0.731181 \\ 8 & 1 & 0 & 1.478689 & -2.248691 & 0.621333 \\ 9 & 1 & 0 & 2.439201 & -1.008629 & -0.206568 \\ 10 & 1 & 0 & 2.708860 & -1.340732 & 1.502570 \\ 11 & 6 & 0 & -1.729763 & 0.418172 & -1.781255 \\ 12 & 1 & 0 & -1.909728 & 1.457605 & -1.491754 \\ 13 & 1 & 0 & -1.720293 & 0.360117 & -2.876357 \\ 14 & 1 & 0 & -2.568530 & -0.176463 & -1.410737 \\ 15 & 6 & 0 & 0.739475 & 0.763105 & -1.868336 \\ 16 & 1 & 0 & 0.681008 & 0.695310 & -2.961258 \\ 17 & 1 & 0 & 0.627701 & 1.817899 & -1.593061 \\ 18 & 1 & 0 & 1.741030 & 0.439111 & -1.573129 \\ 19 & 6 & 0 & 1.595471 & 1.155340 & 1.454318 \\ 20 & 1 & 0 & 2.053335 & 1.575275 & 0.552382 \\ 21 & 1 & 0 & 0.875668 & 1.879231 & 1.846961\end{array}$




$\begin{array}{rrrrrr}22 & 1 & 0 & 2.387974 & 1.025513 & 2.201229 \\ 23 & 6 & 0 & -0.228314 & -1.569191 & -1.696794 \\ 24 & 1 & 0 & 0.753539 & -1.989226 & -1.469075 \\ 25 & 1 & 0 & -0.989418 & -2.202934 & -1.227578 \\ 26 & 1 & 0 & -0.366751 & -1.628084 & -2.783212 \\ 27 & 1 & 0 & 0.515072 & -0.544837 & 2.137068\end{array}$

Iso-propyl Tert-butyl ketone iminoxyl - MLM

$\begin{array}{ccrrrr}\text { Iso-propyl Tert-butyl ketone iminoxyl }- \text { MLM } \\ 1 & 6 & 0 & 1.255342 & -0.171199 & 0.078552 \\ 2 & 6 & 0 & -0.151777 & 0.403424 & -0.145513 \\ 3 & 6 & 0 & -1.483708 & -0.364253 & -0.228016 \\ 4 & 7 & 0 & -0.246149 & 1.687226 & -0.217341 \\ 5 & 8 & 0 & -1.200295 & 2.463178 & -0.374176 \\ 6 & 6 & 0 & -1.448150 & -1.731884 & -0.925673 \\ 7 & 1 & 0 & -1.052685 & -1.660134 & -1.943585 \\ 8 & 1 & 0 & -0.858311 & -2.474386 & -0.379044 \\ 9 & 1 & 0 & -2.471172 & -2.120111 & -0.995480 \\ 10 & 6 & 0 & 2.250419 & 0.971899 & 0.371285 \\ 11 & 1 & 0 & 1.961592 & 1.538297 & 1.262645 \\ 12 & 1 & 0 & 3.248854 & 0.552962 & 0.540732 \\ 13 & 1 & 0 & 2.313981 & 1.673762 & -0.466176 \\ 14 & 6 & 0 & 1.263896 & -1.135034 & 1.287667 \\ 15 & 1 & 0 & 2.278339 & -1.520338 & 1.444702 \\ 16 & 1 & 0 & 0.954564 & -0.621564 & 2.204858 \\ 17 & 1 & 0 & 0.605511 & -1.996161 & 1.141257 \\ 18 & 6 & 0 & -2.147126 & -0.456524 & 1.162871 \\ 19 & 1 & 0 & -1.579579 & -1.101192 & 1.842063 \\ 20 & 1 & 0 & -2.233292 & 0.534566 & 1.619952 \\ 21 & 1 & 0 & -3.157086 & -0.873753 & 1.069828 \\ 22 & 6 & 0 & 1.754331 & -0.919150 & -1.182867 \\ 23 & 1 & 0 & 1.159579 & -1.807472 & -1.407891 \\ 24 & 1 & 0 & 1.732562 & -0.262161 & -2.059543 \\ 25 & 1 & 0 & 2.790376 & -1.245621 & -1.031908 \\ 26 & 1 & 0 & -2.127193 & 0.283623 & -0.837451\end{array}$

Di-tert-butyl ketone Oxime - MLM

$\begin{array}{rrrrrr}1 & 6 & 0 & 1.518175 & -0.070334 & -0.009954 \\ 2 & 6 & 0 & 0.026103 & 0.376513 & -0.050034 \\ 3 & 6 & 0 & -1.211020 & -0.569685 & -0.001869 \\ 4 & 7 & 0 & -0.063724 & 1.660347 & -0.059029 \\ 5 & 8 & 0 & -1.371390 & 2.193870 & -0.019622 \\ 6 & 1 & 0 & -1.184870 & 3.145263 & -0.034596 \\ 7 & 6 & 0 & -0.922915 & -2.086402 & -0.083785 \\ 8 & 1 & 0 & -0.227973 & -2.446313 & 0.678304 \\ 9 & 1 & 0 & -1.870354 & -2.615529 & 0.072279 \\ 10 & 1 & 0 & -0.550326 & -2.386226 & -1.066430 \\ 11 & 6 & 0 & -1.952861 & -0.324748 & 1.339102 \\ 12 & 1 & 0 & -1.333512 & -0.639063 & 2.187512 \\ 13 & 1 & 0 & -2.215523 & 0.725035 & 1.472160 \\ 14 & 1 & 0 & -2.874903 & -0.918717 & 1.361933 \\ 15 & 6 & 0 & 1.870132 & -1.085492 & -1.125613 \\ 16 & 1 & 0 & 1.518283 & -0.737037 & -2.103811 \\ 17 & 1 & 0 & 2.959901 & -1.188157 & -1.185889 \\ 18 & 1 & 0 & 1.464775 & -2.082015 & -0.949792 \\ 19 & 6 & 0 & 2.459405 & 1.142348 & -0.208150 \\ 20 & 1 & 0 & 3.497746 & 0.792363 & -0.162426 \\ 21 & 1 & 0 & 2.300355 & 1.621196 & -1.178928 \\ 22 & 1 & 0 & 2.316572 & 1.902952 & 0.562016 \\ 23 & 6 & 0 & -2.145732 & -0.264638 & -1.203524 \\ 24 & 1 & 0 & -2.507021 & 0.762059 & -1.200470 \\ 25 & 1 & 0 & -1.623499 & -0.444731 & -2.150840 \\ 26 & 1 & 0 & -3.009564 & -0.939863 & -1.167811 \\ 27 & 6 & 0 & 1.846109 & -0.666313 & 1.381741 \\ 28 & 1 & 0 & 1.671481 & 0.076686 & 2.167990 \\ 29 & 1 & 0 & 1.257304 & -1.554910 & 1.621795 \\ 30 & 1 & 0 & 2.903937 & -0.953864 & 1.419704\end{array}$

Di-tert-butyl ketone Iminoxyl - MLM

$\begin{array}{rrrrrr}1 & 6 & 0 & 1.503070 & -0.113827 & -0.009894 \\ 2 & 6 & 0 & 0.029426 & 0.348412 & -0.052981\end{array}$




$\begin{array}{rrrrrr}3 & 6 & 0 & -1.270775 & -0.513446 & -0.003105 \\ 4 & 7 & 0 & -0.125378 & 1.628252 & -0.048011 \\ 5 & 8 & 0 & -1.088967 & 2.407420 & -0.020500 \\ 6 & 6 & 0 & -1.060311 & -2.035955 & -0.125906 \\ 7 & 1 & 0 & -0.390524 & -2.444277 & 0.635018 \\ 8 & 1 & 0 & -2.032756 & -2.524993 & 0.004172 \\ 9 & 1 & 0 & -0.685947 & -2.321995 & -1.112315 \\ 10 & 6 & 0 & -1.970597 & -0.227368 & 1.348021 \\ 11 & 1 & 0 & -1.365072 & -0.585428 & 2.188417 \\ 12 & 1 & 0 & -2.152368 & 0.842508 & 1.485417 \\ 13 & 1 & 0 & -2.937913 & -0.742658 & 1.385000 \\ 14 & 6 & 0 & 1.845009 & -1.102527 & -1.150180 \\ 15 & 1 & 0 & 1.548211 & -0.698305 & -2.124840 \\ 16 & 1 & 0 & 2.928875 & -1.265965 & -1.174294 \\ 17 & 1 & 0 & 1.374596 & -2.078965 & -1.025301 \\ 18 & 6 & 0 & 2.435819 & 1.108110 & -0.173678 \\ 19 & 1 & 0 & 3.478833 & 0.774998 & -0.126375 \\ 20 & 1 & 0 & 2.281172 & 1.606225 & -1.136410 \\ 21 & 1 & 0 & 2.280194 & 1.848613 & 0.616584 \\ 22 & 6 & 0 & -2.195911 & -0.092853 & -1.170297 \\ 23 & 1 & 0 & -2.427358 & 0.974616 & -1.147124 \\ 24 & 1 & 0 & -1.732857 & -0.323315 & -2.136731 \\ 25 & 1 & 0 & -3.140671 & -0.646008 & -1.108804 \\ 26 & 6 & 0 & 1.820925 & -0.757614 & 1.362189 \\ 27 & 1 & 0 & 1.641495 & -0.045572 & 2.175450 \\ 28 & 1 & 0 & 1.224689 & -1.652358 & 1.559072 \\ 29 & 1 & 0 & 2.876848 & -1.051830 & 1.398128\end{array}$

Tert-butyl 1-Adamantyl Ketone Oxime - MLM

$\begin{array}{cccccc}1 & 6 & 0 & 2.914667 & 0.704229 & 1.202234 \\ 2 & 1 & 0 & 2.643144 & 1.368951 & 2.033980 \\ 3 & 1 & 0 & 4.002991 & 0.558806 & 1.250214 \\ 4 & 6 & 0 & 2.518577 & 1.345771 & -0.141553 \\ 5 & 1 & 0 & 3.006228 & 2.325137 & -0.239759 \\ 6 & 6 & 0 & 2.188924 & -0.645676 & 1.344825 \\ 7 & 1 & 0 & 2.454644 & -1.111543 & 2.303397 \\ 8 & 6 & 0 & 0.661822 & -0.419473 & 1.309992 \\ 9 & 1 & 0 & 0.365473 & 0.258969 & 2.121365 \\ 10 & 1 & 0 & 0.156850 & -1.371798 & 1.496822 \\ 11 & 6 & 0 & 0.991352 & 1.553565 & -0.187168 \\ 12 & 1 & 0 & 0.707079 & 2.042907 & -1.125939 \\ 13 & 1 & 0 & 0.687541 & 2.233217 & 0.614785 \\ 14 & 6 & 0 & 0.221355 & 0.203533 & -0.050843 \\ 15 & 6 & 0 & 0.703590 & -0.703636 & -1.220605 \\ 16 & 1 & 0 & 0.218471 & -1.679801 & -1.191367 \\ 17 & 1 & 0 & 0.425633 & -0.236421 & -2.175717 \\ 18 & 6 & 0 & 2.957796 & 0.429506 & -1.297705 \\ 19 & 1 & 0 & 4.046648 & 0.281443 & -1.277504 \\ 20 & 1 & 0 & 2.714827 & 0.893857 & -2.263489 \\ 21 & 6 & 0 & 2.602449 & -1.574806 & 0.184977 \\ 22 & 1 & 0 & 3.683525 & -1.767060 & 0.225216 \\ 23 & 1 & 0 & 2.102288 & -2.548674 & 0.282656 \\ 24 & 6 & 0 & 2.231390 & -0.919774 & -1.161902 \\ 25 & 1 & 0 & 2.523260 & -1.584641 & -1.986037 \\ 26 & 6 & 0 & -1.316366 & 0.436319 & -0.064501 \\ 27 & 6 & 0 & -2.417979 & -0.668701 & 0.000646 \\ 28 & 6 & 0 & -3.147134 & -0.529087 & 1.364198 \\ 29 & 1 & 0 & -3.987406 & -1.233314 & 1.405364 \\ 30 & 1 & 0 & -2.469709 & -0.770813 & 2.191848 \\ 31 & 1 & 0 & -3.534701 & 0.478709 & 1.515822 \\ 32 & 6 & 0 & -1.952263 & -2.140606 & -0.104668 \\ 33 & 1 & 0 & -1.181978 & -2.418508 & 0.617248 \\ 34 & 1 & 0 & -2.821670 & -2.777814 & 0.095866 \\ 35 & 1 & 0 & -1.599583 & -2.396845 & -1.107247 \\ 36 & 6 & 0 & -3.417980 & -0.479452 & -1.172479 \\ 37 & 1 & 0 & -4.177822 & -1.269287 & -1.126046 \\ 38 & 1 & 0 & -3.918928 & 0.486108 & -1.144305 \\ 39 & 1 & 0 & -2.901092 & -0.573425 & -2.135033 \\ 40 & 7 & 0 & -1.584130 & 1.695776 & -0.062156 \\ 41 & 8 & 0 & -2.950491 & 2.047022 & 0.003283\end{array}$




\begin{tabular}{cccrrr}
42 & 1 & 0 & -2.894072 & 3.014962 & -0.006007 \\
\multicolumn{7}{c}{ Tert-butyl 1-Adamantyl } & Ketone Oxime & Z-isomer & - MLM \\
1 & 6 & 0 & 3.009924 & -0.148435 & 0.339520 \\
2 & 1 & 0 & 3.042645 & -0.170851 & 1.437913 \\
3 & 1 & 0 & 4.051122 & -0.167096 & -0.011721 \\
4 & 6 & 0 & 2.310485 & 1.133433 & -0.148158 \\
5 & 1 & 0 & 2.833251 & 2.014109 & 0.249078 \\
6 & 6 & 0 & 2.239242 & -1.370432 & -0.191495 \\
7 & 1 & 0 & 2.725081 & -2.294103 & 0.151424 \\
8 & 6 & 0 & 0.796602 & -1.339214 & 0.349512 \\
9 & 1 & 0 & 0.813954 & -1.344190 & 1.448358 \\
10 & 1 & 0 & 0.257708 & -2.234026 & 0.031309 \\
11 & 6 & 0 & 0.848558 & 1.172419 & 0.356249 \\
12 & 1 & 0 & 0.389740 & 2.094613 & -0.013136 \\
13 & 1 & 0 & 0.858951 & 1.228160 & 1.449246 \\
14 & 6 & 0 & 0.039553 & -0.062090 & -0.140821 \\
15 & 6 & 0 & 0.085631 & 0.011793 & -1.703187 \\
16 & 1 & 0 & -0.488037 & -0.803887 & -2.141664 \\
17 & 1 & 0 & -0.388406 & 0.949842 & -2.025226 \\
18 & 6 & 0 & 2.325652 & 1.171035 & -1.686817 \\
19 & 1 & 0 & 3.360117 & 1.152200 & -2.057676 \\
20 & 1 & 0 & 1.872978 & 2.104903 & -2.048451 \\
21 & 6 & 0 & 2.215404 & -1.340894 & -1.733655 \\
22 & 1 & 0 & 3.239964 & -1.400447 & -2.127066 \\
23 & 1 & 0 & 1.672225 & -2.214455 & -2.118443 \\
24 & 6 & 0 & 1.539998 & -0.040458 & -2.217302 \\
25 & 1 & 0 & 1.523980 & -0.021524 & -3.315499 \\
26 & 6 & 0 & -1.434220 & -0.162663 & 0.346999 \\
27 & 6 & 0 & -2.108533 & 0.744353 & 1.424004 \\
28 & 6 & 0 & -1.465807 & 0.474870 & 2.807555 \\
29 & 1 & 0 & -1.963126 & 1.086250 & 3.570596 \\
30 & 1 & 0 & -1.587331 & -0.577274 & 3.088568 \\
31 & 1 & 0 & -0.400015 & 0.712560 & 2.842112 \\
32 & 6 & 0 & -3.613454 & 0.405992 & 1.561437 \\
33 & 1 & 0 & -3.776934 & -0.634395 & 1.848854 \\
34 & 1 & 0 & -4.047953 & 1.052252 & 2.333706 \\
35 & 1 & 0 & -4.152351 & 0.579305 & 0.625219 \\
36 & 6 & 0 & -2.033646 & 2.253351 & 1.081396 \\
37 & 1 & 0 & -2.732113 & 2.801320 & 1.724488 \\
38 & 1 & 0 & -1.046712 & 2.688154 & 1.241284 \\
39 & 1 & 0 & -2.329404 & 2.439765 & 0.042304 \\
40 & 7 & 0 & -2.251542 & -1.082044 & -0.034421 \\
41 & 8 & 0 & -1.746871 & -2.035808 & -0.947506 \\
42 & 1 & 0 & -2.525909 & -2.598773 & -1.076007
\end{tabular}

Tert-butyl 1-Adamantyl Ketone Iminoxyl - MLM

$\begin{array}{cccccc}1 & 6 & 0 & 2.880487 & 0.689734 & 1.212431 \\ 2 & 1 & 0 & 2.598663 & 1.338516 & 2.053190 \\ 3 & 1 & 0 & 3.970543 & 0.559991 & 1.259710 \\ 4 & 6 & 0 & 2.479527 & 1.346134 & -0.123166 \\ 5 & 1 & 0 & 2.957242 & 2.331113 & -0.208411 \\ 6 & 6 & 0 & 2.175907 & -0.674026 & 1.335519 \\ 7 & 1 & 0 & 2.448125 & -1.148175 & 2.287919 \\ 8 & 6 & 0 & 0.645808 & -0.471132 & 1.300570 \\ 9 & 1 & 0 & 0.333991 & 0.180626 & 2.127742 \\ 10 & 1 & 0 & 0.151723 & -1.436128 & 1.454973 \\ 11 & 6 & 0 & 0.950354 & 1.535256 & -0.167439 \\ 12 & 1 & 0 & 0.661810 & 2.030127 & -1.103352 \\ 13 & 1 & 0 & 0.637519 & 2.199517 & 0.647099 \\ 14 & 6 & 0 & 0.202479 & 0.174255 & -0.048067 \\ 15 & 6 & 0 & 0.688815 & -0.719058 & -1.226062 \\ 16 & 1 & 0 & 0.210935 & -1.700616 & -1.193985 \\ 17 & 1 & 0 & 0.398795 & -0.251940 & -2.177413 \\ 18 & 6 & 0 & 2.925149 & 0.448922 & -1.292976 \\ 19 & 1 & 0 & 4.015726 & 0.315990 & -1.277240 \\ 20 & 1 & 0 & 2.673653 & 0.922859 & -2.251770 \\ 21 & 6 & 0 & 2.604539 & -1.580464 & 0.163359 \\ 22 & 1 & 0 & 3.688788 & -1.753864 & 0.199499 \\ 23 & 1 & 0 & 2.122114 & -2.564284 & 0.248804\end{array}$




$\begin{array}{rrrrrr}24 & 6 & 0 & 2.219589 & -0.914026 & -1.173820 \\ 25 & 1 & 0 & 2.520124 & -1.562172 & -2.007803 \\ 26 & 6 & 0 & -1.319559 & 0.410360 & -0.068248 \\ 27 & 6 & 0 & -2.482837 & -0.630863 & 0.000240 \\ 28 & 6 & 0 & -3.179615 & -0.457868 & 1.372388 \\ 29 & 1 & 0 & -4.065094 & -1.102737 & 1.427109 \\ 30 & 1 & 0 & -2.508119 & -0.736123 & 2.192609 \\ 31 & 1 & 0 & -3.504600 & 0.575056 & 1.527747 \\ 32 & 6 & 0 & -2.071120 & -2.110184 & -0.148048 \\ 33 & 1 & 0 & -1.317976 & -2.428089 & 0.576605 \\ 34 & 1 & 0 & -2.961401 & -2.727612 & 0.020221 \\ 35 & 1 & 0 & -1.707800 & -2.339803 & -1.153519 \\ 36 & 6 & 0 & -3.490310 & -0.335281 & -1.137133 \\ 37 & 1 & 0 & -4.338005 & -1.027053 & -1.065366 \\ 38 & 1 & 0 & -3.881124 & 0.683567 & -1.087613 \\ 39 & 1 & 0 & -3.021723 & -0.476562 & -2.117918 \\ 40 & 7 & 0 & -1.661988 & 1.654002 & -0.051827 \\ 41 & 8 & 0 & -2.728159 & 2.283653 & -0.001667\end{array}$

Tert-butyl 1-Adamantyl Ketone Iminoxyl Z-isomer - MLM

$\begin{array}{rrrrrr}1 & 6 & 0 & 3.004174 & -0.177055 & 0.324283 \\ 2 & 1 & 0 & 3.039211 & -0.188017 & 1.422707 \\ 3 & 1 & 0 & 4.044178 & -0.210381 & -0.028498 \\ 4 & 6 & 0 & 2.319325 & 1.109585 & -0.174412 \\ 5 & 1 & 0 & 2.858720 & 1.987088 & 0.206653 \\ 6 & 6 & 0 & 2.223068 & -1.399737 & -0.193481 \\ 7 & 1 & 0 & 2.700161 & -2.323327 & 0.160479 \\ 8 & 6 & 0 & 0.780335 & -1.343917 & 0.346726 \\ 9 & 1 & 0 & 0.792094 & -1.360447 & 1.445038 \\ 10 & 1 & 0 & 0.222410 & -2.227438 & 0.016423 \\ 11 & 6 & 0 & 0.862954 & 1.174475 & 0.343894 \\ 12 & 1 & 0 & 0.404492 & 2.097765 & -0.025759 \\ 13 & 1 & 0 & 0.877688 & 1.231237 & 1.437445 \\ 14 & 6 & 0 & 0.050912 & -0.055816 & -0.142002 \\ 15 & 6 & 0 & 0.078432 & -0.021348 & -1.699702 \\ 16 & 1 & 0 & -0.499192 & -0.857199 & -2.106057 \\ 17 & 1 & 0 & -0.406095 & 0.901994 & -2.046369 \\ 18 & 6 & 0 & 2.319471 & 1.128277 & -1.714194 \\ 19 & 1 & 0 & 3.350338 & 1.101125 & -2.093557 \\ 20 & 1 & 0 & 1.867830 & 2.059895 & -2.082603 \\ 21 & 6 & 0 & 2.198899 & -1.385371 & -1.735266 \\ 22 & 1 & 0 & 3.222603 & -1.452034 & -2.128925 \\ 23 & 1 & 0 & 1.652839 & -2.260558 & -2.112135 \\ 24 & 6 & 0 & 1.526869 & -0.087418 & -2.228332 \\ 25 & 1 & 0 & 1.502421 & -0.078969 & -3.326054 \\ 26 & 6 & 0 & -1.423006 & -0.125482 & 0.352447 \\ 27 & 6 & 0 & -2.128716 & 0.727101 & 1.432501 \\ 28 & 6 & 0 & -1.498232 & 0.458553 & 2.821320 \\ 29 & 1 & 0 & -2.024323 & 1.041961 & 3.586830 \\ 30 & 1 & 0 & -1.583542 & -0.601030 & 3.087180 \\ 31 & 1 & 0 & -0.441531 & 0.734539 & 2.867569 \\ 32 & 6 & 0 & -3.619524 & 0.326555 & 1.518321 \\ 33 & 1 & 0 & -3.744040 & -0.730988 & 1.769173 \\ 34 & 1 & 0 & -4.107600 & 0.920727 & 2.299280 \\ 35 & 1 & 0 & -4.140809 & 0.512280 & 0.573564 \\ 36 & 6 & 0 & -2.086237 & 2.242016 & 1.117255 \\ 37 & 1 & 0 & -2.758425 & 2.772317 & 1.802159 \\ 38 & 1 & 0 & -1.093483 & 2.678012 & 1.239447 \\ 39 & 1 & 0 & -2.427711 & 2.442628 & 0.095205 \\ 40 & 7 & 0 & -2.150827 & -1.078353 & -0.122896 \\ 41 & 8 & 0 & -1.988098 & -2.008152 & -0.926884\end{array}$


Table S5. Calculated structures from Figure 1 and text.

\begin{tabular}{cccrrr}
\multicolumn{6}{c}{ Linearized di-tert-butyl ketone iminoxyl radical } \\
1 & 6 & 0 & 1.398246 & -0.356030 & -0.002197 \\
2 & 6 & 0 & 0.000003 & 0.349355 & -0.000020 \\
3 & 6 & 0 & -1.398239 & -0.356031 & 0.002198 \\
4 & 7 & 0 & -0.000007 & 1.613865 & -0.000010 \\
5 & 8 & 0 & -0.000014 & 2.838515 & -0.000007 \\
6 & 6 & 0 & -1.466907 & -1.555743 & -0.966041 \\
7 & 1 & 0 & -0.775997 & -2.360563 & -0.702096 \\
8 & 1 & 0 & -2.478801 & -1.978005 & -0.943491 \\
9 & 1 & 0 & -1.259397 & -1.249594 & -1.997001 \\
10 & 6 & 0 & -1.751439 & -0.820360 & 1.435837 \\
11 & 1 & 0 & -1.077892 & -1.598184 & 1.803567 \\
12 & 1 & 0 & -1.715352 & 0.020792 & 2.137138 \\
13 & 1 & 0 & -2.769784 & -1.228414 & 1.453194 \\
14 & 6 & 0 & 1.751301 & -0.820780 & -1.435742 \\
15 & 1 & 0 & 1.715122 & 0.020169 & -2.137283 \\
16 & 1 & 0 & 2.769656 & -1.228811 & -1.453079 \\
17 & 1 & 0 & 1.077744 & -1.598737 & -1.803179 \\
18 & 6 & 0 & 2.467694 & 0.664410 & 0.443365 \\
19 & 1 & 0 & 3.454537 & 0.188075 & 0.441922 \\
20 & 1 & 0 & 2.510364 & 1.529630 & -0.225448 \\
21 & 1 & 0 & 2.270539 & 1.033280 & 1.455998 \\
22 & 6 & 0 & -2.467641 & 0.664267 & -0.443789 \\
23 & 1 & 0 & -2.510392 & 1.529696 & 0.224749 \\
24 & 1 & 0 & -2.270388 & 1.032834 & -1.456514 \\
25 & 1 & 0 & -3.454481 & 0.187925 & -0.442311 \\
26 & 6 & 0 & 1.467004 & -1.555451 & 0.966397 \\
27 & 1 & 0 & 1.259532 & -1.249016 & 1.997280 \\
28 & 1 & 0 & 0.776111 & -2.360376 & 0.702722 \\
29 & 1 & 0 & 2.478908 & -1.977692 & 0.943919
\end{tabular}

Perpendicular di-tert-butyl ketone iminoxyl radical

$\begin{array}{rlrrrr}1 & 8 & 0 & -0.068711 & 2.671461 & 0.157259 \\ 2 & 7 & 0 & -0.037919 & 1.587745 & -0.485095 \\ 3 & 6 & 0 & 0.004815 & 0.318007 & -0.178853 \\ 4 & 6 & 0 & -1.374399 & -0.368920 & -0.015228 \\ 5 & 6 & 0 & 1.400880 & -0.328363 & -0.020946 \\ 6 & 6 & 0 & 1.503261 & -1.056584 & 1.343008 \\ 7 & 1 & 0 & 0.801627 & -1.888389 & 1.440265 \\ 8 & 1 & 0 & 2.514196 & -1.467393 & 1.448594 \\ 9 & 1 & 0 & 1.335913 & -0.365769 & 2.176112 \\ 10 & 6 & 0 & 1.711345 & -1.319360 & -1.169786 \\ 11 & 1 & 0 & 1.054164 & -2.191610 & -1.167844 \\ 12 & 1 & 0 & 1.622280 & -0.824764 & -2.143508 \\ 13 & 1 & 0 & 2.742666 & -1.679958 & -1.071073 \\ 14 & 6 & 0 & -1.771628 & -0.131667 & 1.469041 \\ 15 & 1 & 0 & -1.693446 & 0.930092 & 1.727156 \\ 16 & 1 & 0 & -2.811626 & -0.444204 & 1.623079 \\ 17 & 1 & 0 & -1.144200 & -0.702177 & 2.159741 \\ 18 & 6 & 0 & -2.414586 & 0.348233 & -0.907498 \\ 19 & 1 & 0 & -3.397941 & -0.114690 & -0.766716 \\ 20 & 1 & 0 & -2.494697 & 1.409456 & -0.659052 \\ 21 & 1 & 0 & -2.148036 & 0.267354 & -1.967063 \\ 22 & 6 & 0 & 2.476273 & 0.779057 & -0.043504 \\ 23 & 1 & 0 & 2.502331 & 1.290679 & -1.010864 \\ 24 & 1 & 0 & 2.295005 & 1.534869 & 0.726165 \\ 25 & 1 & 0 & 3.462129 & 0.334374 & 0.132446 \\ 26 & 6 & 0 & -1.435961 & -1.873082 & -0.345590 \\ 27 & 1 & 0 & -1.220729 & -2.060894 & -1.402798 \\ 28 & 1 & 0 & -0.753821 & -2.478646 & 0.256308 \\ 29 & 1 & 0 & -2.450689 & -2.238154 & -0.147220\end{array}$

Linearized acetone iminoxyl radical

$\begin{array}{rrrrrr}1 & 6 & 0 & -1.127080 & 1.300932 & -0.000025 \\ 2 & 1 & 0 & -1.775806 & 1.357749 & -0.883856 \\ 3 & 1 & 0 & -1.776158 & 1.357575 & 0.883560 \\ 4 & 1 & 0 & -0.459497 & 2.165656 & 0.000193\end{array}$




$\begin{array}{cccccc}5 & 6 & 0 & -0.341321 & -0.000010 & -0.000053 \\ 6 & 6 & 0 & -1.127153 & -1.300883 & -0.000030 \\ 7 & 1 & 0 & -1.776683 & -1.357188 & -0.883298 \\ 8 & 1 & 0 & -0.459623 & -2.165655 & -0.000910 \\ 9 & 1 & 0 & -1.775403 & -1.358024 & 0.884132 \\ 10 & 7 & 0 & 0.920483 & -0.000029 & 0.000286 \\ 11 & 8 & 0 & 2.144140 & -0.000018 & -0.000147\end{array}$

Perpendicular acetone iminoxyl radical

$\begin{array}{cccrrr}1 & 8 & 0 & 1.999074 & -0.000084 & 0.259991 \\ 2 & 7 & 0 & 0.939148 & -0.000058 & -0.423137 \\ 3 & 6 & 0 & -0.334224 & -0.000038 & -0.115124 \\ 4 & 6 & 0 & -1.076011 & -1.297634 & 0.064289 \\ 5 & 6 & 0 & -1.075777 & 1.297754 & 0.064266 \\ 6 & 1 & 0 & -1.921106 & -1.408523 & -0.629493 \\ 7 & 1 & 0 & -0.405860 & -2.152061 & -0.049288 \\ 8 & 1 & 0 & -1.498811 & -1.311945 & 1.079466 \\ 9 & 1 & 0 & -1.497708 & 1.312605 & 1.079815 \\ 10 & 1 & 0 & -0.405600 & 2.152032 & -0.050243 \\ 11 & 1 & 0 & -1.921470 & 1.408479 & -0.628814\end{array}$


Table S6. Raw data for the calculated gas phase O-C, N-O and N-H bond dissociation enthalpies (BDEs) at $298 \mathrm{~K}$ found in Table 3.

\begin{tabular}{|c|c|c|c|c|c|c|c|c|c|}
\hline & & & parent & & & radical & & $\begin{array}{c}\text { H-atom/ } \\
\text { Benzyl/ } \\
\text { Benzyloxyl }\end{array}$ & \\
\hline$\underline{R}, R^{\prime}$ & Bond & Eel & Hvib & $\mathrm{H}(298 \mathrm{~K})$ & Eel & Hvib & $\mathrm{H}(298 \mathrm{~K})$ & $\mathrm{H}(298 \mathrm{~K})$ & BDE \\
\hline $\mathrm{Me}, \mathrm{Me}$ & $\mathrm{O}-\mathrm{C}$ & -520.527859 & 0.222948 & -520.3091628 & -248.5863026 & 0.095492 & -248.4925898 & -271.739061 & 48.6 \\
\hline $\mathrm{Me}, \mathrm{Me}$ & $\mathrm{N}-\mathrm{O}$ & -520.527859 & 0.222948 & -520.3091628 & -173.2268208 & 0.089523 & -173.1389612 & -347.0885947 & 51.2 \\
\hline $\mathrm{Me}, \mathrm{Me}$ & $\mathrm{N}-\mathrm{H}$ & -173.3384872 & 0.103404 & -173.2370159 & -172.6841106 & 0.090183 & -172.5956039 & -0.497638 & 90.2 \\
\hline $\mathrm{Ph}, \mathrm{Ph}$ & $\mathrm{O}-\mathrm{C}$ & -905.2286109 & 0.335791 & -904.8992609 & -633.2916701 & 0.208295 & -633.0873427 & -271.739061 & 45.7 \\
\hline $\mathrm{Ph}, \mathrm{Ph}$ & $\mathrm{N}-\mathrm{O}$ & -905.2286109 & 0.335791 & -904.8992609 & -557.9286002 & 0.202547 & -557.7299093 & -347.0885947 & 50.7 \\
\hline $\mathrm{Ph}, \mathrm{Ph}$ & $\mathrm{N}-\mathrm{H}$ & -556.9144638 & 0.216194 & -556.7023906 & -556.2576455 & 0.202885 & -556.0586231 & -0.497638 & 91.7 \\
\hline 9-fluorenyl & $\mathrm{O}-\mathrm{C}$ & -903.9994233 & 0.31321 & -903.6922162 & -632.061142 & 0.185511 & -631.8791566 & -271.739061 & 46.4 \\
\hline 9-fluorenyl & $\mathrm{N}-\mathrm{O}$ & -903.9994233 & 0.31321 & -903.6922162 & -556.6944273 & 0.179702 & -556.5181382 & -347.0885947 & 53.6 \\
\hline 9-fluorenyl & $\mathrm{N}-\mathrm{H}$ & -555.7149855 & 0.192964 & -555.5256917 & -555.0571732 & 0.179831 & -554.8807576 & -0.497638 & 92.4 \\
\hline $\operatorname{Pr}, \operatorname{Pr}$ & $\mathrm{O}-\mathrm{C}$ & -678.4040721 & 0.341909 & -678.0687228 & -406.4652084 & 0.214242 & -406.2550493 & -271.739061 & 46.8 \\
\hline $\operatorname{Pr}, \operatorname{Pr}$ & $\mathrm{N}-\mathrm{O}$ & -678.4040721 & 0.341909 & -678.0687228 & -331.1060062 & 0.208387 & -330.9015886 & -347.0885947 & 49.3 \\
\hline $\operatorname{Pr}, \mathrm{Pr}$ & $\mathrm{N}-\mathrm{H}$ & -330.6372162 & 0.223157 & -330.4183151 & -329.9837357 & 0.20989 & -329.7778443 & -0.497638 & 89.6 \\
\hline $\mathrm{Pr}, \mathrm{Bu}$ & $\mathrm{O}-\mathrm{C}$ & -717.8682209 & 0.371152 & -717.5041959 & -445.9307549 & 0.243384 & -445.6920192 & -271.739061 & 45.9 \\
\hline $\mathrm{Pr}, \mathrm{Bu}$ & $\mathrm{N}-\mathrm{O}$ & -717.8682209 & 0.371152 & -717.5041959 & -370.5705445 & 0.237389 & -370.3376876 & -347.0885947 & 48.9 \\
\hline $\mathrm{Pr}, \mathrm{Bu}$ & $\mathrm{N}-\mathrm{H}$ & -369.953064 & 0.252542 & -369.705348 & -369.302126 & 0.239215 & -369.0674785 & -0.497638 & 88.0 \\
\hline $\mathrm{Bu}, \mathrm{Bu}$ & $\mathrm{O}-\mathrm{C}$ & -757.3283336 & 0.400183 & -756.9358408 & -485.3970929 & 0.272336 & -485.1299669 & -271.739061 & 41.9 \\
\hline $\mathrm{Bu}, \mathrm{Bu}$ & $\mathrm{N}-\mathrm{O}$ & -757.3283336 & 0.400183 & -756.9358408 & -410.0388926 & 0.266471 & -409.7775178 & -347.0885947 & 43.8 \\
\hline $\mathrm{Bu}, \mathrm{Bu}$ & $\mathrm{N}-\mathrm{H}$ & -409.2729957 & 0.282122 & -408.9962735 & -408.6241903 & 0.268569 & -408.3607582 & -0.497638 & 86.5 \\
\hline $\mathrm{Bu}, \mathrm{Ad}$ & $\mathrm{O}-\mathrm{C}$ & -990.4235267 & 0.513398 & -989.9200153 & -718.49288 & 0.385699 & -718.1145903 & -271.739061 & 41.6 \\
\hline Z-isomer & $\mathrm{O}-\mathrm{C}$ & -990.422588 & 0.513369 & -989.919105 & -718.493324 & 0.385865 & -718.1148715 & -271.739061 & 40.9 \\
\hline $\mathrm{Bu}, \mathrm{Ad}$ & $\mathrm{N}-\mathrm{O}$ & -990.4235267 & 0.513398 & -989.9200153 & -643.1350312 & 0.379542 & -642.762779 & -347.0885947 & 43.1 \\
\hline Z-isomer & $\mathrm{N}-\mathrm{O}$ & -990.422588 & 0.513369 & -989.919105 & -643.1350312 & 0.379542 & -642.762779 & -347.0885947 & 42.5 \\
\hline $\mathrm{Bu}, \mathrm{Ad}$ & $\mathrm{N}-\mathrm{H}$ & -641.5981096 & 0.395457 & -641.2102512 & -640.949777 & 0.381766 & -640.575344 & -0.497638 & 86.1 \\
\hline Z-isomer & $\mathrm{N}-\mathrm{H}$ & -641.5982602 & 0.395474 & -641.2103851 & -640.949777 & 0.381766 & -640.575344 & -0.497638 & 86.2 \\
\hline benzyl & & & & & -271.857979 & 0.121196 & -271.739061 & & \\
\hline benzyloxyl & & & & & -347.2123344 & 0.126113 & -347.0885947 & & \\
\hline
\end{tabular}


Table S7. Raw data for the calculated gas phase O-H bond dissociation enthalpies (BDEs) at $298 \mathrm{~K}$ found in Table 4.

\begin{tabular}{lcccr}
\hline & parent & radical & H-atom & \\
& $\mathrm{H}(298 \mathrm{~K})$ & $\mathrm{H}(298 \mathrm{~K})$ & $\mathrm{H}(298 \mathrm{~K})$ & \multicolumn{2}{c}{$\mathrm{BDE}$} \\
\hline G3MP2 & -248.064819 & -247.427450 & -0.499478 & 86.5 \\
G3 & -248.255316 & -247.619779 & -0.498642 & 85.9 \\
CBS-QB3 & -248.033672 & -247.400639 & -0.497457 & 85.1 \\
CBS-APNO & -248.344593 & -247.712254 & -0.497585 & 84.6 \\
DFT & see next Table & & & \\
\hline
\end{tabular}

Table S8. Characterization of the stationary points of the three geometries of di-tert-butyliminoxyl radical shown in Figure 1.

\begin{tabular}{lcccrrr}
\hline geometry & Im frequency? & Eel & Hvib & H(298K) & deltaEel & deltaH \\
A. minimum energy & n/a & -483.842383 & 0.274379 & -483.573254 & 0 & 0.0 \\
B. linearized & -410.8791 & -483.825800 & 0.272808 & -483.558211 & 10.4 & 9.4 \\
C. perpendicular & -547.6155 & -483.798289 & 0.271888 & -483.531602 & 27.7 & 26.1 \\
\hline
\end{tabular}

Table S9. Characterization of the stationary points of the three geometries of dimethyliminoxyl radical as discussed in the text.

\begin{tabular}{|c|c|c|c|c|c|c|}
\hline geometry & Im frequency? & Eel & Hvib & $\mathrm{H}(298 \mathrm{~K})$ & deltaEel & deltaH \\
\hline A. minimum energy & $\mathrm{n} / \mathrm{a}$ & -247.904310 & 0.096057 & -247.810043 & 0 & 0.0 \\
\hline B. linearized & -472.0205 & -247.883450 & 0.094507 & -247.790703 & 13.1 & 12.1 \\
\hline C. perpendicular & -622.4568 & -247.852286 & 0.092999 & -247.761018 & 32.6 & 30.8 \\
\hline
\end{tabular}


Table S10. Raw data for the calculated gas phase O-H bond dissociation enthalpies (BDEs) at $298 \mathrm{~K}$ found in Table 5.

\begin{tabular}{|c|c|c|c|c|c|c|c|c|c|}
\hline \multirow[b]{2}{*}{$\mathrm{R}, \mathrm{R}^{\prime}$} & & \multicolumn{3}{|c|}{ parent } & \multicolumn{3}{|c|}{ radical } & \multicolumn{2}{|l|}{$\mathrm{H}$-atom } \\
\hline & & Eel & Hvib & $\mathrm{H}(298 \mathrm{~K})$ & Eel & Hvib & $\mathrm{H}(298 \mathrm{~K})$ & $\mathrm{H}(298 \mathrm{~K})$ & $\mathrm{BDE}$ \\
\hline $\mathrm{H}, \mathrm{H}$ & DFT & -169.877631 & 0.049065 & -169.829444 & -169.237581 & 0.036292 & -169.201920 & -0.497638 & 81.5 \\
\hline $\mathrm{H}, \mathrm{H}$ & CBS & & & -169.569127 & & & -168.938716 & -0.497457 & 83.4 \\
\hline $\mathrm{H}, \mathrm{Me}$ & DFT & -209.212771 & 0.078801 & -209.135426 & -208.570536 & 0.066198 & -208.505549 & -0.497638 & 83.0 \\
\hline Z-isomer & DFT & -209.212075 & 0.079029 & -209.134506 & -208.573645 & 0.066491 & -208.508371 & -0.497638 & 80.6 \\
\hline $\mathrm{H}, \mathrm{Me}$ & CBS & & & -208.801046 & & & -208.168156 & -0.497457 & 85.0 \\
\hline Z-isomer & CBS & & & -208.800267 & & & -208.169407 & -0.497457 & 83.7 \\
\hline $\mathrm{H}, \mathrm{CF} 3$ & DFT & -507.031083 & 0.057732 & -506.974397 & -506.389659 & 0.045009 & -506.345450 & -0.497638 & 82.4 \\
\hline Z-isomer & DFT & -507.026882 & 0.057875 & -506.970056 & -506.388186 & 0.045319 & -506.343673 & -0.497638 & 80.8 \\
\hline $\mathrm{H}, \mathrm{CF} 3$ & CBS & & & -506.314794 & & & -505.682622 & -0.497457 & 84.5 \\
\hline Z-isomer & CBS & & & -506.311554 & & & -505.681368 & -0.497457 & 83.3 \\
\hline $\mathrm{Me}, \mathrm{Me}$ & DFT & -248.545834 & 0.108483 & -248.439382 & -247.904310 & 0.096057 & -247.810043 & -0.497638 & 82.6 \\
\hline $\mathrm{Me}, \mathrm{Me}$ & CBS & see previous Table & & & & & & & \\
\hline $\mathrm{H}, \mathrm{Ph}$ & DFT & -401.004363 & 0.135196 & -400.871717 & -400.363666 & 0.122425 & -400.243543 & -0.497638 & 81.9 \\
\hline Z-isomer & DFT & -401.001159 & 0.135671 & -400.868047 & -400.367594 & 0.122874 & -400.247031 & -0.497638 & 77.4 \\
\hline $\mathrm{H}, \mathrm{Ph}$ & CBS & & & -400.190344 & & & -399.556949 & -0.497457 & 85.3 \\
\hline Z-isomer & CBS & & & -400.187242 & & & -399.559692 & -0.497457 & 81.6 \\
\hline $\mathrm{Me}, \mathrm{Ph}$ & DFT & -440.333641 & 0.165039 & -440.171730 & -439.694786 & 0.152376 & -439.545293 & -0.497638 & 80.8 \\
\hline Z-isomer & DFT & -440.329342 & 0.165012 & -440.167458 & -439.694987 & 0.152562 & -439.545312 & -0.497638 & 78.1 \\
\hline $\mathrm{Me}, \mathrm{Ph}$ & CBS & & & -439.420819 & & & -438.788738 & -0.497457 & 84.5 \\
\hline Z-isomer & CBS & & & -439.417238 & & & -438.789606 & -0.497457 & 81.7 \\
\hline $\mathrm{Ph}, \mathrm{Ph}$ & DFT & -632.117962 & 0.220958 & -631.901217 & -631.481269 & 0.208563 & -631.276679 & -0.497638 & 79.6 \\
\hline 9-fluorenyl & DFT & -630.922136 & 0.198262 & -630.727647 & -630.284884 & 0.185620 & -630.102792 & -0.497638 & 79.8 \\
\hline Et, Et & DFT & -327.195441 & 0.168813 & -327.029830 & -326.555142 & 0.156319 & -326.401782 & -0.497638 & 81.8 \\
\hline Et, Et & CBS & & & -326.481416 & & & -325.849762 & -0.497457 & 84.2 \\
\hline $\operatorname{Pr}, \operatorname{Pr}$ & DFT & -405.842299 & 0.228192 & -405.618460 & -405.203814 & 0.215644 & -404.992280 & -0.497638 & 80.7 \\
\hline $\operatorname{Pr}, \operatorname{Pr}$ & CBS & & & -404.931595 & & & -404.302690 & -0.497457 & 82.5 \\
\hline $\mathrm{Bu}, \mathrm{Pr}$ & DFT & -445.159651 & 0.257756 & -444.906822 & -444.522848 & 0.245099 & -444.282431 & -0.497638 & 79.5 \\
\hline $\mathrm{Bu}, \mathrm{Bu}$ & DFT & -484.472876 & 0.286934 & -484.191435 & -483.842383 & 0.274379 & -483.573254 & -0.497638 & 75.6 \\
\hline $\mathrm{Bu}, \mathrm{Ad}$ & DFT & -716.798023 & 0.400422 & -716.405296 & -716.168199 & 0.387817 & -715.787832 & -0.497638 & 75.2 \\
\hline Z-isomer & DFT & -716.797743 & 0.400580 & -716.404861 & -716.168367 & 0.387982 & -715.787839 & -0.497638 & 74.9 \\
\hline
\end{tabular}

